\title{
Origin and evolution of the atmospheres of early Venus, Earth and Mars
}

\author{
Helmut Lammer ${ }^{1}$, Aubrey L. Zerkle ${ }^{2}$, Stefanie \\ Gebauer $^{3}$, Nicola Tosi ${ }^{4,5}$, Lena Noack ${ }^{6}$, Manuel \\ Scherf $^{1}$, Elke Pilat-Lohinger ${ }^{7}$, Manuel Güdel ${ }^{7}$, \\ John Lee Grenfell ${ }^{3}$, Mareike Godolt ${ }^{5}$, Athanasia \\ Nikolaou $^{4,5}$
}

Received: March 2017/ Accepted: date

\begin{abstract}
The origin and evolution of the atmospheres of Earth, Venus and Mars are reviewed from the time when their protoplanets were released from the protoplanetary disk a few million years after the Sun came into being. The early disk-embedded phase of the evolution of protoplanetary cores that can accumulate gas from the disk and form thin planetary $\mathrm{H}_{2}$-envelopes is also discussed. This scenario is compared to cases of late stage planet formation, where terrestrial planets accrete from large planetary embryos after the protoplanetary disk already disappeared. The differences between these two scenarios are discussed by investigating non-radiogenic noble gas isotope anomalies observed in the present atmospheres of the three planets. The role of the efficiency of the young Sun's EUV radiation and solar wind to the escape of early atmospheres is also discussed. The catastrophic outgassing of volatiles and the formation and cooling of steam atmospheres after the solidification of magma oceans is addressed together with the geochemical evidence of additional delivery of volatile-rich chondritic materials during the main stages of planetary formation. Unlike early Venus and Earth, no nebula-based $\mathrm{H}_{2}$-envelope could be accumulated on early Mars due to its low planetary mass. According to the young Sun's luminosity and EUV flux history, Mars' magma ocean related outgassed steam atmosphere could have been lost during the first hundred Myrs. Depending on the young Sun's EUV flux, the presence of greenhouse gases, impacts, and the amount of greenhouse gases outgassed additional to that from the magma ocean, Mars could have developed episodically standing bodies of liquid water

H. Lammer

Austrian Academy of Sciences, Space Research Institute, Schmiedlstr. 6, A-8042, Graz, Austria

Tel.: +433164120641

Fax: +433164120690

E-mail: helmut.lammer@oeaw.ac.at

${ }^{1}$ Austrian Academy of Science, Space Research Institute, Graz, Austria

${ }^{2}$ School of Earth \& Environmental Sciences and Centre for Exoplanet Science, University of St. Andrews, St. Andrews, Fife, UK

${ }^{3}$ German Aerospace Center, Institute for Planetary Research, Department of Extrasolar Planets and Atmospheres, Berlin Germany

${ }^{4}$ German Aerospace Center, Institute for Planetary Research, Department of Planetary Physics, Berlin Germany

${ }^{5}$ Technical University of Berlin, Department of Astronomy and Astrophysics, Berlin, Germany

${ }^{6}$ Freie Universität Berlin, Department of Earth Sciences, Berlin Germany

${ }^{7}$ University of Vienna, Department of Astrophysics, Vienna, Austria
\end{abstract}


during the pre-Noachian. An atmosphere of up to several hundred mbar was most likely lost by a complex interplay of various nonthermal atmospheric escape processes, impacts, carbonate precipitation, and serpentinization during the past 3.5 - 4.0 Gyr ago. On early Earth, the complex interplay between the atmosphere-ocean-surface-interaction related $\mathrm{CO}_{2}$ carbonate-silicate cycle and surface weathering of atmospheric constituents is discussed. The evolution scenario of early Earth is then compared with the atmospheric evolution of stagnant-lid type planets like Venus and Mars during the first few hundred Myrs. The diversity between early Earth, Venus and Mars, which is related to their differing geochemical, geodynamical and geophysical conditions, including plate tectonics, crust and mantle oxidation processes, the role of $\mathrm{NH}_{4}^{+}$speciation in upper mantle fluids and the evolution of outgassed secondary $\mathrm{N}_{2}$ atmospheres is investigated. The relevant factors related to the solar EUV flux evolution during Earth's Archean epoch, where a fraction of Xe escaped via the polar caps and the outgassed $\mathrm{N}_{2}$ atmosphere was most likely slightly enriched in ${ }^{15} \mathrm{~N}$ isotopes by atmospheric escape compared to the primordial chondritic-based mantle nitrogen, is discussed. The atmospheric conditions when the earliest life forms on Earth originated, the role of the Faint Young Sun Paradox (FYSP), greenhouse gases, and the related build up of atmospheric $\mathrm{N}_{2}$ and $\mathrm{O}_{2}$ up to and during the Great Oxidation Event (GOE) $\approx 2.3$ Gyr ago are also addressed. The review concludes with a discussion on the implications of understanding Earth's geophysical and related atmospheric evolution in relation to the discovery of potential habitable terrestrial exoplanets.

Keywords primordial atmospheres $\cdot$ secondary atmospheres $\cdot$ atmospheric evolution $\cdot$ early Earth, Venus, Mars · habitability

\section{Introduction}

Lammer et al. (2009) reviewed the most important astrophysical and geophysical factors, which are thought to be relevant for the evolution of potential liquid water bearing planetary habitats. So far Earth is the only known example of an inhabited planet, which is populated by a large diversity of life forms. Compared to Venus and Mars and also the icy satellites, modern Earth is unique in the sense that it has surface liquid water, active plate tectonics, a magnetic dynamo (which it has possessed for billions of years (Gyr)), and an $\mathrm{N}_{2}$-dominated atmosphere with a greenhouse effect that keeps its surface from freezing. Following Lammer et al. (2009), Earth represents a perfect habitat (Class I), which is one of the drivers to understand the main factors which enabled Earth's atmosphere to evolve from probably an $\mathrm{H}_{2}$ - to a $\mathrm{CO}_{2}$ - (Venus-like), and finally to an $\mathrm{N}_{2}$-dominated, while Venus and Mars ended with a $\mathrm{CO}_{2}$ atmosphere.

In the cases of Venus and Earth, the growth time of their protoplanetary cores (e.g., Yu and Jacobsen 2011) determined whether they originated after the nebular gas evaporated, or whether they grew to larger masses while they were embedded in the nebula, in which $\mathrm{H}_{2}$ could accumulate before the protoplanetary disk disappeared (e.g., Ikoma and Genda 2006; Raymond et al. 2006, 2009; Lunine et al. 2011; Ikoma and Hori 2012; Morbidelli et al. 2010, 2012; Walsh et al. 2011; O’Brien et al. 2014; Stökl et al. 2015, 2016).

Due to the huge number of exoplanets confirmed in recent years (e.g., Pollacco et al. 2006; Bakos et al. 2004; Auvergne et al. 2009; Borucki et al. 2010; Howe et al. 2014), a large population of planets with masses slightly larger than Solar System terrestrial planets but with over-sized radii, indicate that nebular-based $\mathrm{H}_{2}$-envelopes are a common phenomenon on small planets (Mullally et al. 2015; Rogers 2015; Lammer et al. 2016; Cubillos et al. 2017; Fossati et al. 2017). 
There are arguments that point to the fact that significant amounts of noble gases have been trapped from the protoplanetary disk, and left in solar composition in the interiors of early Venus and Earth (e.g. Mizuno et al. 1980; Sasaki and Nakazawa 1989; Porcelli and Pepin 2000; Dixon et al. 2000; Porcelli et al. 2001; Becker et al. 2003; Halliday 2004; Yokochi and Marty 2004). Solar-like isotopes, which are embedded in the nebular gas, can enter the planetary interior via magma oceans that formed below the accumulated $\mathrm{H}_{2}$-envelopes (e.g., Porcelli and Pepin 2003; Ikoma and Genda 2006; Marty 2012; Stökl et al. 2015; 2016), when a growing protoplanet with masses $>0.5 M_{\text {Earth }}$ is embedded in the gas disk (Sekiya et al. 1980a, 1980b; Sasaki and Nakazawa 1988; Harper and Jacobsen 1996; Porcelli et al. 1998; Pepin 2000; Stökl et al. 2016). Related to the protoplanetary mass and the EUV activity of the young Sun, captured $\mathrm{H}_{2}$-envelopes of low mass cores can later be lost due to hydrodynamic escape (e.g., Erkaev et al. 2013; Johnstone et al. 2015a; Lammer et al. 2014; 2016; Luger et al. 2015; Owen and Mohanty 2016; Fossati et al. 2017).

During the first Gyr the young Sun's EUV flux was several tens to hundreds of times higher than today's solar value (e.g. Ribas et al. 2005; Claire et al. 2012; Tu et al. 2015), which is very important for the evolution of early planetary atmospheres, including the loss of nebular-captured $\mathrm{H}_{2}$ envelopes (Tu et al. 2015; Johnstone et al. 2015a). In addition to the possible mechanisms which arise due to captured nebula gas during the early accretion phase, the terrestrial planets also obtained a large amount of volatiles via impactors that originated in the outer Solar System. After their nebular gas was mainly lost to space, volatiles have been outgassed during the solidification of early magma oceans (e.g., Elkins-Tanton 2008; 2012).

Recent ruthenium $(\mathrm{Ru})$ isotope studies (Fischer-Gödde and Kleine 2017) related to primitive meteoritic material indicate that the terrestrial planets obtained the majority of their volatiles $\left(\mathrm{H}_{2} \mathrm{O}, \mathrm{CO}_{2}, \mathrm{CH}_{4}, \mathrm{NH}_{3}, \mathrm{HCN}\right.$, $\left(\mathrm{N}_{2}\right)$, etc.) via impactors of volatile-rich bodies from the outer Solar System during the main stages of terrestrial planet formation, and not as previously expected during the late veneer (e.g., Albaréde 2009; Kleine 2011). This recent finding is also in agreement with the analysis of chalcophile elements in Martian meteorites, which indicate that impactors on Mars during the late veneer were depleted in volatiles (Wang and Becker 2016). From magma ocean studies it is expected that at some point in their history all three planets outgassed volatiles, which on Earth and Venus have most likely been dominated by $\mathrm{H}_{2} \mathrm{O}$ and $\mathrm{CO}_{2}$ (e.g., Elkins-Tanton 2008, 2011; Marcq 2012; Hamano et al. 2013; Lebrun et al. 2013; Massol et al. 2016; Salvador et al. 2017).

Table 1 shows the abundance of the main atmospheric species of present Venus, Earth and Mars. Venus' atmospheric $\mathbf{C O}_{2}$ inventory corresponds to $\approx 90$ bar partial pressure, which is slightly higher than the $\mathrm{CO}_{2}$ inventory in Earth's surface (e.g., Walker 1985). The terrestrial carbonate-silicate cycle weathered out the $\mathrm{CO}_{2}$ during the Hadean eon (e.g., Walker 1985; Kasting 1993; Sleep and Zahnle 2001), so that $\approx 3.0-4.0 \mathrm{Gyr}$ ago $\mathrm{N}_{2}$ started to become the main atmospheric species. Several geophysical and geochemical studies of early Earth's nitrogen inventory (atmosphere and interior), including the volcanic release of $\mathrm{N}_{2}$ during the Archean, indicate that the $\mathrm{N}_{2}$ build-up of early secondary atmospheres on terrestrial planets is strongly connected to geophysical processes that lead to the oxidation of the crust and upper mantle, as will be discussed in the following sections (e.g., Busigny and Bebout 2013; Mikhail and Sverjensky 2014; Wordsworth 2016a; Zerkle and Mikhail 2017).

Understanding the origin and evolution of the atmospheres of Venus, Earth and Mars and why Earth's atmosphere evolved differently compared to those of its neighboring planets, so that life could arise and evolve, is crucial for the search of life and atmospheric bio-markers on potentially habitable terrestrial exoplanets (e.g., Fridlund et al. 2010). The present review therefore aims to piece together the latest findings in astrophysics, planetary formation, 
Table 1 Main atmospheric species present in Venus' (Oyamo et al. 1980), Earth's (NOAA Earth System Research Laboratory: https://www.esrl.noaa.gov/) and Mars' ((Mahaffy et al. 2013) atmospheres. ${ }^{\dagger}$ An equivalent amount of about $60-80$ bar of $\mathrm{CO}_{2}$ is located in Earth's crust in the form of carbonates (e.g., Ronov and Yaroshevsky 1967; Holland 1978; Walker 1985, Kasting 1993).

\begin{tabular}{l|lll}
\hline Species & Venus & Earth & Mars \\
\hline $\mathrm{CO}_{2}$ & $96.5 \%$ & $0.0407 \% \dagger$ & $95.97 \%$ \\
$\mathrm{~N}_{2}$ & $3.5 \%$ & $78.084 \%$ & $\mathbf{1 . 8 9 \%}$ \\
$\mathrm{O}_{2}$ & $<<$ & $20.946 \%$ & $0.146 \%$ \\
$\mathrm{Ar}$ & $70 \mathrm{ppm}$ & $0.9340 \%$ & $1.93 \%$ \\
$\mathrm{SO}_{2}$ & $150 \mathrm{ppm}$ & - & - \\
\hline
\end{tabular}

geophysics and atmosphere evolution, to understand why early Earth evolved to a Class I habitat and why Venus and Mars did not. In Sect. 2 the radiation and solar wind of the young Sun is briefly discussed. Sect. 3 focuses on the first 100 Myr after the Solar System formed and will describe the latest formation hypotheses of the three planets, and the most likely hypothesis on how Venus, Earth, and Mars obtained their earliest atmospheres. In Sect. 4 the loss of $\mathrm{CO}_{2}$ and $\mathrm{N}_{2}$ to surface sinks and space on one-plate planets like Venus or Mars are compared to Earth, where at least some form of proto-plate tectonics operated early on. Sect. 5 discusses the origin of $\mathrm{N}_{2}$-dominated atmospheres, the nitrogen cycle on present Earth, the $\mathrm{N}_{2}$-speciation during the Hadean eon, the planet's oxidation states and the rise of an $\mathrm{N}_{2}$ atmosphere during the Archean. Sect. 6 addresses the response of higher EUV fluxes of the young Sun against Earth's early $\mathrm{N}_{2}$-dominated upper atmosphere, including a discussion on the observed ${ }^{14} \mathrm{~N} /{ }^{15} \mathrm{~N}$ isotope disequilibrium in the atmosphere/crust/mantle and the escape of Xe. Sect. 7 investigates possible greenhouse gases, the production of prebiotic molecules and the modification of Earth's atmosphere after life originated during the late Hadean and early Archean eons. After summarizing possible reasons why early Earth evolved into an $\mathrm{N}_{2}$-dominated atmosphere contrary to Venus and Mars about 3.5 Gyr ago, the makings of Earth-like habitats and the implications for the search of Earth-like exoplanets are summarized in Sect. 8. Finally, Sect. 9 concludes the review.

\section{The young Sun}

While the long-term evolution of the Sun's bolometric radiation is quite well understood, following from calculations of the Sun's internal structure and nuclear reactions (e.g., Sackmann and Boothroyd 2003), the evolution of the high-energy radiation is less clear. The Sun's magnetic activity declined during its main-sequence lifetime, and its photospheric luminosity was $\approx 30 \%$ lower when the Sun arrived on the main sequence compared to present-day levels (e.g., Güdel 2007). The emission shortward of $\approx 150-200 \mathrm{~nm}$ originates in magnetically active regions at the solar chromospheric, transition region, and coronal level (in order of increasing height and temperature). The heating of the plasma in these regions may indeed be due to the dissipation of magnetic energy.

How the long-term evolution of the magnetically induced high-energy radiation is related to solar/stellar rotation started being clarified in the late sixties. Kraft (1967) realized that stellar chromospheric emission is stronger in younger stars that also rotate more rapidly. Kraft (1967) therefore proposed long-term spin-down due to a magnetized wind. This was put on a theoretical basis by Weber and Davis (1967), formulating the magnetic torques. 


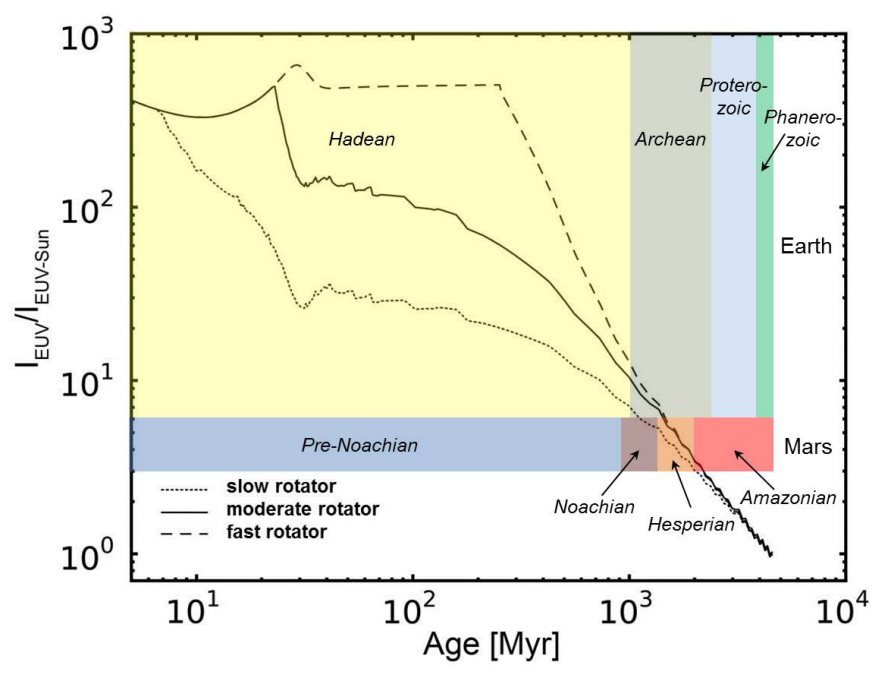

Fig. 1 Expected rotation-based EUV enhancement factor normalized to that of today's solar level (after Tu et al. 2015). The dotted line corresponds to the EUV activity evolution enhancement of slowly rotating weakly active young solar-like stars. The solid line corresponds to a young Sun rotating moderately, while the dashed line represents the evolution of a fast rotating EUV active young solar-like star. The shaded areas mark the different geological eons on Earth and Mars.

Skumanich (1972) showed that empirically, both the stellar rotation rate and chromospheric emission decay as the square root of time and implied that surface magnetic fields follow the same decay law. In stellar astronomy, the activity-rotation-age relations became established with ultraviolet (see, e.g., Zahnle and Walker 1982) and X-ray observations (e.g., Pallavicini et al. 1981, Walter 1981) from space. It consists of three important relations.

First, stellar activity, for example as expressed by the total coronal X-ray luminosity, follows a decay law with increasing rotation period $P_{\text {rot }}$ of the form $L_{X} \propto P^{-2 \ldots 2.7}$ for a given stellar mass on the main sequence. This suggests that the internal dynamo strongly relates to the surface rotation period (e.g., Pallavicini et al. 1981, Walter 1981, Maggio et al. 1987, Güdel et al. 1997, Ayres 1997, Wright et al. 2011). However, for rotation periods as short as a couple of days (depending on stellar mass), the X-ray luminosity saturates at $L_{\mathrm{X} \text {, sat }} \approx 10^{-3} L_{\text {bol }}$ (see, e.g., Wright et al. 2011). The cause for this saturation is not understood, but could be related to surface saturation of the magnetic flux, or some internal threshold of the magnetic dynamo. A unified relation for the activity-rotation relation was presented by Pizzolato et al. (2003) for all spectral types on the cool main sequence.

Second, activity decays with age. The decay laws can be reconstructed from open cluster observations in X-rays. On average, one finds $L_{\mathrm{X}} \propto t^{-1.5}$ for solar analogs (Maggio et al. 1987, Güdel et al. 1997). Obviously, this is related to stellar spin-down. The third ingredient, the relation between stellar spin-down and age (Skumanich 1972) is also well studied based on coeval cluster samples. On average, one finds a relation of the form $P_{\text {rot }} \propto t^{0.6}$ (e.g., Ayres 1997 for solar analogs), compatible with the above two relations. The activity decay law has often been used for (exo)planetary atmospheric loss calculations based on simple fits to observed trends using limited samples. For X-rays, the "Sun in Time" sample was used with ages between $\approx 100 \mathrm{Myr}$ and $\approx 5$ Gyr (Güdel et al. 1997), and this was complemented with the corresponding decay laws for the extreme-ultraviolet and far ultraviolet radiation 
(Ribas et al. 2005) and also near-UV (Claire et al. 2012). Generally, the decay in time is steeper for higher-energetic radiation, which therefore also decays by a larger factor over a given time. Using the average regression laws referred to above, X-rays decrease by a factor of $\approx 1000-2000$ over the main-sequence life of a Sun-like star, EUV by a few hundred, and UV by factors of a few tens (e.g., Ribas et al. 2005).

However, this approach is not accurate because in reality the rotation behavior of young stars ( $<$ a few hundred Myr for solar analogs) is highly non-unique. Cluster samples show a wide dispersion in rotation periods for ages up to a few hundred Myr, after which they gradually converge to a unique, stellar-mass dependent value (Soderblom et al. 1993). This convergence is attributed to the feedback between the magnetic dynamo and angular momentum loss in a magnetized wind. The wide dispersion of $P_{\text {rot }}$ for young stars instead reflects the initial conditions for rotation starting after the protostellar disk phase (e.g., Gallet and Bouvier 2013).

An evolutionary decay law for high-energy radiation therefore needs to account for the dispersion of rotation periods. Observationally, a wide distribution of $L_{X}$ in young clusters was in fact known from early cluster surveys (e.g., Stauffer et al. 1994). A proper analysis of the problem was laid out in the work by Johnstone et al. (2015a, 2015b) and Tu et al. (2015) in which a solar-wind model was adapted to stars at different activity levels and different magnetic fluxes, fitting distributions of $P_{\text {rot }}$ in time from various clusters. Translating rotation to high-energy radiation, Tu et al. (2015) reported the finding illustrated in Fig. 1. This figure shows that, depending on whether a solar analog starts out as a slow or fast rotator after the disk phase, the X-ray and EUV evolutionary tracks first diverge of (a slow rotator rapidly decays with time while that of a fast rotator does not) and then converge again after several hundred Myr when the rotation periods converge. The nearly constant X-ray and EUV luminosity for fast (but spinning-down) rotators is due to the saturation effect.

The distribution of high-energy radiation is broadest in the range of a few tens to a few hundreds of Myr, precisely the range of interest for protoatmospheric loss, the formation of a secondary atmosphere, a crust and a liquid water ocean on Earth, and the earliest steps toward the formation of life. A proper study of atmospheric evolution therefore needs to account for the uncertainty of early stellar high-energy evolution. To the present day, we do not know the evolutionary track in high-energy radiation the Sun has taken.

Moreover, one further parameter that is important for atmospheric modeling, that is, the hardness of high-energy radiation. Specifically, the X-ray hardness (the relative amount of "harder" to "softer" radiation) decreases with decreasing X-ray surface flux, because more active stars are dominated by hotter coronal plasma (Johnstone and Güdel 2015). This has consequences for the irradiating spectra because the penetration depth of radiation in an atmosphere depends on photon energy.

\section{The first 100 Myr: From planetary embryos to protoplanets}

The wide range of possibilities which exist regarding the nature of the earliest atmospheres provides a wide spectrum of consequences for thermal and magmatic evolution of planetary surfaces and mantles, as well as the onset for the outgassing of secondary atmospheres. A brief description on the latest formation hypothesis of Venus, Earth and Mars and how these planets obtained their earliest atmospheres during the first $100 \mathrm{Myr}$ are outlined below. 


\subsection{Formation of Venus, Earth and Mars}

Contrary to Venus and Earth, Mars has only about one tenth of Earth's mass and seems therefore to be a remaining large planetary embryo that obviously never became a fullygrown planet. This fact is the reason of a long-standing problem in dynamical models that were used to reconstruct the formation of the terrestrial planets in the Solar System. In the classical core-accretion scenario (Bodenheimer and Pollack 1986; Pollack et al. 1996) the formation of planets begins on the smallest scale and lasts from sub-micron sized dust up to Jupiter-like planets (e.g., O'Brien et al. 2014; Johansen et al. 2014). The ideas of Safronov (1969) and Wetherill (1980) reflect the early stages where dust accumulates into larger bodies (i.e., planetesimals) due to a gravitational instability in a thin dust layer and the subsequent coagulation of the planetesimals leads to the so-called planetary embryos, which are Moon to Mars-sized bodies. Collisions of embryos yield finally to the formation of terrestrial planets.

That the formation of terrestrial planets may take place over a period of up to $\approx 100$ - 200 Myr was shown in many models using the classical core-accretion process (see e.g. Chambers and Wetherill 1998; Chambers 2001; Raymond et al. 2004; 2005; 2006; 2007; O'Brien et al. 2006). However, the formation of Mars constituted a major problem for these studies - refereed to as the "small Mars" problem (see e.g. Wetherill 1991; Chambers 2001; Raymond et al. 2009). A possible solution has been provided by Hansen (2009), whereby the planets form in a narrow annulus of large planetary embryos between 0.7 and $1 \mathrm{AU}$. As Mercury and Mars were scattered beyond the edges of the annulus, they could not grow as large as Earth and Venus. This evidence is supported by a study of Dauphas and Pourmand (2011) that is based on Thorium/Tungsten (Th/W) and Thorium/Hafnium (Th/Hf) ratios in the Martian mantle, which confirm that the growth time of proto-Mars was of the order of a few Myr, well within the upper limit of $\approx 10$ Myr as suggested by Hansen (2009).

Taking into account the fact that planets are not born at the locations where we observe them today, Walsh et al.(2011) showed that giant planet migration could help to restrict the terrestrial planet formation to the area between 0.7 and $1 \mathrm{AU}$. This model hypothesis is known as "Grand-Tack" scenario (Walsh et al. 2011) and provides so far the most promising results describing the evolution of the Solar System from planet formation up to the final architecture of our planetary system.

The most important features of this hypothesis are that Jupiter formed first a massive $\geq 10 M_{\text {Earth }}$ core and accreted gas from the disk (e.g., Pollack et al. 1996). This is now confirmed by NASA's JUNO spacecraft that measured Jupiter's low-order gravitational moments and found that Jupiter's core contains $\approx 7-25 M_{\text {Earth }}$ of heavy elements (Wahl et al. 2017). When the planet reached a Jupiter-mass it opened a gap in the disk and migrated inwards with a velocity depending on the disk's local viscous timescale (Lin and Papaloizou 1986). Farther out, Saturn was growing at the same time albeit somewhat slower. After the accretion of gas, Saturn migrated also inward but faster than Jupiter (e.g., Masset and Papaloizou 2003) and entered into a 2:3 mean motion resonance with Jupiter (Masset and Snellgrove 2001; Morbidelli and Crida 2007; Pierens and Nelson 2008). For a truncation of the embryo disk at $1 \mathrm{AU}$, Jupiter has to be located at $\approx 1.5 \mathrm{AU}$ which defines also the "tack point" where the direction of migration changes and both planets migrate outwards, probably until the gas disk is dissipated. Recent simulations by Brasser et al. (2016) moved the tack point to $\approx 2 \mathrm{AU}$. However, in both cases the two giant planets influence terrestrial planet formation which continues after the dispersal of the gas disk. The most promising model for this late stage of solar system formation is the so-called "Nice model" (Tsiganis et al, 2005). To prevent strong perturbations of Jupiter and Saturn during terrestrial planet formation, the "Nice model" (Tsiganis et al. 2005; Morbidelli et al. 2007) has been modified 
with a so-called "jumping Jupiter" (Levison et al. 2011) that moves the giant planets quite quickly into the final orbit.

Recently Brasser et al. (2017) studied a scenario where Mars originated outside of the feeding zone of Earth in the asteroid belt during primary accretion. In such a scenario Mars always remained significantly farther from the Sun than Earth and its growth was stunted early so that its mass remained relatively low. According to this study which is also based on the Grand Tack model, such a formation scenario had a probability of $\approx 2 \%$ in the model runs. However, Brasser et al. (2017) suggest that Mars' formation required a specific dynamical pathway, which was less valid for Earth and Venus. In the studied formation scenario Mars' volatile budget is most likely different from Venus and Earth, which formed close enough that one can expect that both planets obtained a nearly identical composition from common building blocks. However, one should keep in mind that although the "Grand Tack" model provides good results for the early formation of the Solar System planets, its associated assumptions lead to criticisms as discussed in Raymond and Morbidelli 2014, which requires further studies.

Important implications of the briefly described truncation mechanism are the scattering of primitive planetesimals onto planet-crossing orbits during the early formation of the planets, and a much faster accretion time of proto-Venus and Earth (e.g., Walsh et al. 2011). Most model runs lead to the occurrence of the final giant impacts typically earlier than $\approx 30 \mathrm{Myr}$, and a substantial amount of mass is accreted after that event. This timescale is in agreement with those estimated from ${ }^{142} \mathrm{Nd}$ isotopic data (Boyet and Carlson 2005) and Hf-W isotope chronometry of meteoritic material, which predicts a short time of $\approx 10 \mathrm{Myr}$ for the main growth stage of terrestrial planets in the Solar System (e.g., Lugmair and Shukolyukow 1998; Nyquist et al. 2001; Yin et al. 2002; Kleine et al. 2002). According to Hf-W isotope analysis, the earliest time the Moon-forming giant impact could have taken place, corresponds to the end of main-stage accretion at approximately $30 \mathrm{Myr}$ (Kleine et al. 2002; Jacobsen 2005;

Wood et al. 2006, Yu and Jacobsen 2011).

However, N-body simulations and Hf-W and Rubidium-Strontium (Rb-Sr) isotope chronology of some Lunar rocks have been used to argue for a later formation of the Moon at 52 to $\geq 100$ Myr (Jacobson et al. 2014). Yu and Jacobsen (2011) explained this discrepancy of the so-called metal-silicate disequilibrium during giant impacts by including constraints from partitioning of refractory siderophile elements $(\mathrm{Ni}, \mathrm{Co}, \mathrm{W}, \mathrm{V}$, and $\mathrm{Nb})$ during core formation. These authors found that if the Moon-forming giant impact occurred late $(\geq 52$ Myr) the main stage of the Earth's accretion should be completed rapidly in $<10.7 \pm 2.5 \mathrm{Myr}$. These however represent two end member solutions and a continuum exists in between these extremes.

One should also point out two processes that produce chemical compositional changes after the solar nebula/chondrite stage of terrestrial planet formation. First, collisional erosion, and post-nebula volatilization. The latter affects "moderately volatile elements", including the radiogenic heat-producing element Potassium $\left({ }^{40} \mathrm{~K}\right)$. Collisional erosion caused by large impactors such as that which was involved in the Moon-forming event are also critical to the eventual budget of the three heat-producing elements (Th, $\mathrm{U}$ and $\mathrm{K}$ ) in the evolution of terrestrial planets (e.g. O'Neill and Palme 2008; Campbell and O'Neill 2012; Jellinek and Jackson 2015; O'Brien et al. 2017) and do also alter the metal-silicate ratio of differentiated bodies of originally chondritic composition (e.g. Bonsor et al. 2014; Carter et al. 2015). 


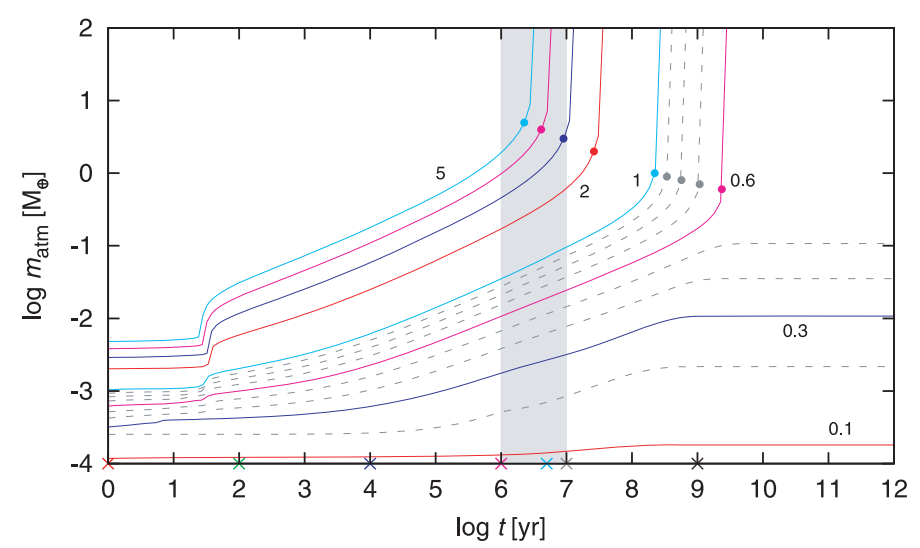

Fig. 2 Total mass of accumulated $\mathrm{H}_{2}$-envelopes within the Hill sphere as a function of time according to protoatmosphere evolution simulations by Stökl et al. (2016). The lines correspond to protoplanetary core masses of $0.1,0.2,0.3,0.4,0.5,0.6,0.7,0.8,0.9,1.0,2.0,3.0,4.0$, and 5.0 $M_{\text {Earth }}$ The typical lifetime of circumstellar disks is illustrated by the shaded area. The filled circles on the lines indicate the point where the atmospheric mass equals the core mass (crossover mass). The crosses along the time axis indicate simulation times, in years, of 1.0 (red), 100.0 (green), $10^{4}$ (blue), $10^{5}$ (purple), $5 \times 10^{6}$ (cyan), $10^{7}$ (gray), and $10^{9}$ (black).

\subsection{Primordial atmospheres}

As briefly discussed above, according to $\mathrm{Hf}-\mathrm{W}$ and ${ }^{142} \mathrm{Nd}$ isotope data (e.g., Boyet and Carlson 2005; Yu and Jacobsen 2011) and in the most recently suggested formation models of the Solar System (e.g., Walsh et al. 2011; O'Brien et al. 2014), proto-Venus, and protoEarth could have grown to $\approx \mathbf{0 . 5}-\mathbf{0 . 7 5} M_{\text {Earth }}$ in the nebula-embedded phase ( $<10 \mathrm{Myr}$ ). If so, both planets may have obtained their final masses after the evaporation of the gas disk from further collisions with planetesimals and planetary embryos within the first 100 Myr (e.g. Kokubo and Ida 2000; Raymond et al. 2004, 2009, 2014; Lunine et al. 2011; Walsh et al. 2011; Morbidelli et al. 2012; O'Brien et al. 2014). During gaseous accretion, the growing protoplanets, gravitationally attract the ambient nebular gas, which forms a solar-type atmosphere. According to various studies, for cores with masses $\geq \mathbf{0 . 5} M_{\text {Earth }}$, gravitational energy, short-lived radioactive elements together with captured $\mathrm{H}_{2}$-envelopes enhance the temperature of the planetary surface to $\geq 2000 \mathrm{~K}$, resulting in deep global magma oceans (e.g., Mizuno et al. 1980; Mizuno and Wetherill 1984; Sasaki and Nakazawa 1989; Ikoma et al. 2000; Ikoma and Genda 2006; Albarède and Blichert-Toft 2007; Bouhifd and Jephcoat 2011; Ikoma and Hori 2012; Broeg and Benz 2012; Stökl et al. 2016).

That Earth-size planetary cores can quickly accrete within the lifetime of the gas disk, i.e. within $\approx 1.0-10.0$ Myr (e.g., Montmerle et al. 2006; Hillenbrand 2008) is now also supported by the discoveries of low mass $\mathrm{H}_{2}$-dominated sub-Neptune-type exoplanets (e.g., Borucki et al. 2010; Howe et al. 2014; Mullally et al. 2015; Cubillos et al. 2017). Careful analysis of the obtained statistics of confirmed exoplanets indicates that most of the small-radius planets, found so far possess an extended $\mathrm{H}_{2}$-envelope (e.g., Rogers 2015; Cubillos et al. 2017).

Fig. 2 shows the total atmospheric mass of accumulated $\mathrm{H}_{2}$-envelopes as a function of time in planetary evolution simulations with core masses between $0.1-5 M_{\text {Earth }}$ (Stökl et al. 2016). The sudden kink in atmosphere mass at about 10.0 and 100.0 years corresponds to the settling of the atmospheric structure into a quasi-hydrostatic configuration. One can see that this effect is more efficient for massive planetary cores. 
In the case that proto-Venus and proto-Earth accreted a mass between $\approx \mathbf{0 . 5 - 0 . 7 5} M_{\text {Earth }}$ during the gas disk lifetime, then the atmospheric growth would come to a halt as soon as the gas disk evaporates. Depending on the nebular lifetime, these protoplanetary cores would have captured thin $\mathrm{H}_{2}$-envelopes between about $0.001-0.1 M_{\text {Earth }}$. More massive protoplanetary cores at some point enter into a hydrogen runaway collapse, while less massive cores eventually settle into a stationary solution. The filled circles on the lines in Fig. 2 indicate the point where the captured hydrogen gas mass equals the mass of the protoplanetary core. According to planet formation models this so-called crossover mass serves as a rough estimate for the onset of gravitational instability and the formation of Jupiter-like gas giants (e.g., Bodenheimer and Pollack 1986; Ikoma et al. 2000).

One should also note that protoplanets which are embedded in the gas disk with masses $>0.3 M_{\text {Earth }}$, which accumulate $\mathrm{H}_{2}$-envelopes can also produce water through oxidation on its magmatic surface and the surrounding atmospheric hydrogen (Ikoma and Genda 2006). Model simulations of Ikoma and Genda (2006) indicate that a small amount of nebular gas is enough on an Earth-like planet to produce an equivalent amount of water comparable to an Earth ocean. Depending on the amount of Fe-bearing minerals, although some water may have been produced by this oxidation process, one should keep in mind that the $\mathrm{D} / \mathrm{H}$ ratio in present Earth's ocean points to delivery by geologically undifferentiated carbonaceous chondrites as the main origin mechanism (e.g., Marty 2012; Bouvier and Boyet 2016) and not via the solar nebula with its $\approx 7$ times lower D/H ratio (e.g., Drake and Richter 2002).

Nebular-based $\mathrm{H}_{2}$-envelopes have been considered earlier to be a fundamental feature of the early Earth (e.g., Mizuno et al. 1980; Sekiya et al. 1980a; 1980b; Mizuno and Wetherill 1984; Sasaki and Nakazawa 1988; Pepin 2000; Porcelli et al. 1998). The importance of such accumulated nebular-based $\mathrm{H}_{2}$-envelopes around large protoplanetary cores and their role in the reproduction of the observed fractionation of noble gases in Venus' and Earth's atmospheres is discussed below.

\subsection{Steam atmosphere outgassing during magma ocean solidification}

Regardless of whether terrestrial planets accumulate a thin $\mathrm{H}_{2}$-envelope or accrete most of their mass after the nebular gas disappeared, the next important stage in the evolution of the early atmosphere is the possible formation of a thick steam atmosphere associated with magma ocean solidification.

The energy sources available during the formation of planetesimals, embryos and, ultimately, planets can be sufficient to raise the temperature of silicate rocks above the liquidus and generate large magma ponds or even deep magma oceans that can be global in nature, as illustrated in Fig. 3, and involve part or even the entire silicate mantle (e.g. Urey 1955; Safronov 1969; Wetherill 1980; LaTourrette and Wasserburg 1998; Solomatov 2000; Halliday et al. 2001; Reese and Solomatov 2006; Albarède and Blichert-Toft 2007; Bouhifd and Jephcoat 2011; Elkins-Tanton 2008, 2011, 2012). Heating caused by the decay of shortlived radiogenic isotopes active during the first few million years of the solar system, ${ }^{26} \mathrm{Al}$ in particular (Castillo-Rogez et al. 2009), can cause melting of planetesimals and the formation of internal magma oceans beneath a solid crust (e.g. Neumann et al., 2014). For planetary embryos and planets that accreted after the first 2-3 Myr of the solar system, magma oceans can form as a result of the conversion of gravitational potential energy into heat upon metalsilicate separation and core formation (e.g. Rubie et al. 2015), and of the dissipation of the kinetic energy of highly energetic accretionary impacts (e.g. Tonks and Melosh 1993; Canup 2004). 
Large-scale melting associated with magma oceans controls the early compositional differentiation of the interior and affects the initial volatile budget of the planetary body (e.g., Albarède and Blichert-Toft 2007; Elkins-Tanton 2008, 2012; Hier-Majumder and Hirschmann 2017).

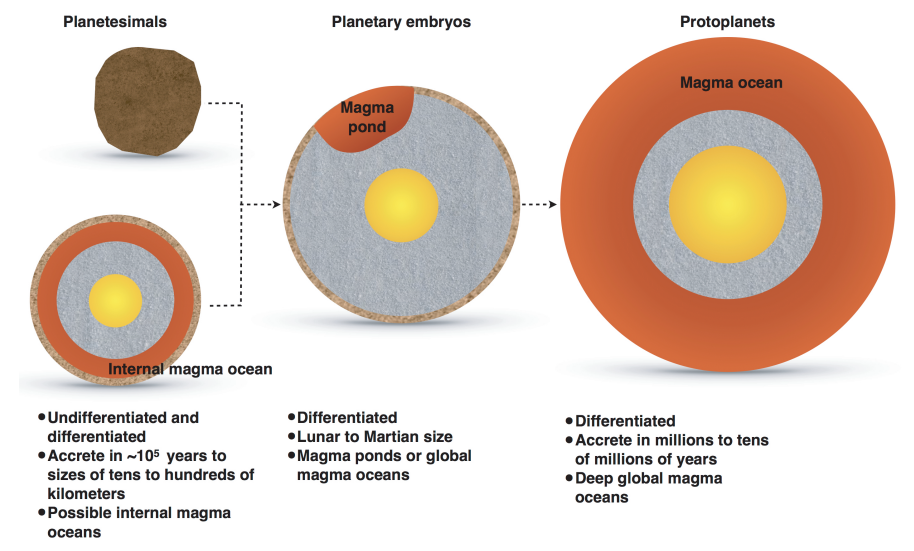

Fig. 3 Illustration of planetestimals and planetary embryos that accrete to protoplanets in a few up to tens of Myr (e.g., Safronov 1969; Wetherill 1980; Elkins-Tanton 2012).

Silicate liquids have a low viscosity in the range between $\approx 0.01-1 \mathrm{~Pa}$ s at the conditions typical of magma oceans (e.g. Karki and Stixrude 2010), which are thus expected to undergo turbulent convection and to cool and crystallize rapidly (e.g. Abe 1997; Solomatov 2000). Since the slope of the adiabatic temperature profile that likely characterizes a turbulent and well-mixed magma ocean is steeper than the slopes of the liquidus and solidus, the solidification of a magma ocean likely begins at its bottom and proceeds upwards (e.g. Walker et al. 1975; Solomatov 2000; Elkins-Tanton 2012). The deeper the magma ocean, the more pronounced this effect. Upon solidification, magma oceans tend to undergo compositional fractionation, with fundamental consequences for the differentiation and long-term thermochemical evolution of the solid mantle (Elkins-Tanton et al. 2003; Tosi et al. 2013; Plesa et al. 2014; Ballmer et al. 2017; Maurice et al. 2017).

In particular, volatile elements, being highly incompatible in mantle minerals, are preferentially enriched in the liquid phase. As a consequence, while the solidification front (i.e. the bottom of the magma ocean) moves toward the surface, the liquid magma ocean progressively becomes more and more enriched in volatiles. If a given volatile species exceeds its solubility in the residual silicate liquid, it is outgassed (e.g. Elkins-Tanton 2008; Lebrun et al. 2013; Salvador et al. 2017). Volatiles that are poorly soluble like $\mathrm{CO}_{2}$ are outgassed as soon as the magma ocean begins to crystallize. More soluble species like $\mathrm{H}_{2} \mathrm{O}$ become supersaturated mostly only toward the end of the solidification, when vast amounts of water vapor are quickly released into the atmosphere leading to a so-called "catastrophic outgassing" as illustrated in Fig. 4.

During planet formation, water, carbon and nitrogen bearing materials are delivered via volatile-rich planetesimals - of chondritic composition (e.g., Drake and Righter 2002; 


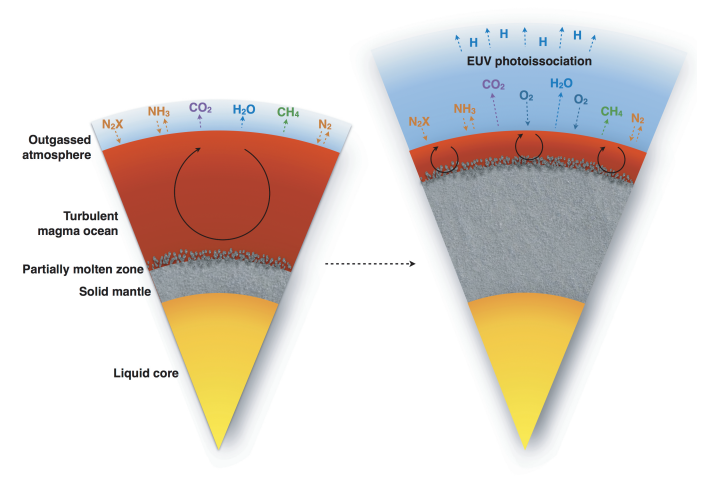

Fig. 4 Illustration of a global convecting magma ocean (left) that solidifies (right) and catastrophically outgasses volatiles that build up a dense steam atmosphere. According to Wordsworth (2016a), abiotic reduction of atmospheric nitrogen should have been effective via direct dissolution of reduced species into the magma ocean or extremely hot surface temperature environments.

Alexander et al. 2012; Marty 2012; Bouvier and Boyet 2016) - from the outer Solar System to accreting protoplanets (e.g. Morbidelli et al. 2000; Raymond et al. 2009; Marty 2012). The extent and composition of the secondary atmosphere generated by magma ocean outgassing ultimately depend on the initial concentration of such volatiles in the accreted body. Volatiles that can be outgassed include $\mathrm{H}_{2} \mathrm{O}$, carbon compounds (e.g., $\mathrm{CO}_{2}, \mathrm{CH}_{4}, \mathrm{CO}$ ), and $\mathrm{N}_{2}$ originating from $\mathrm{NH}_{3}$ (e.g. Zahnle et al. 2010; Elkins-Tanton 2012; Lebrun et al. 2013; Erkaev et al. 2014; Lichtenegger et al. 2016; Massol et al. 2016; Wordsworth 2016a; Salvador et al. 2017; Odert et al. 2018). Highly reduced gases such as $\mathrm{CH}_{4}$ or $\mathrm{NH}_{3}$ were likely present only in low concentrations and may have represented just about a few tenths of percent of the total budget of the outgassed material (e.g., Cloud 1968; Holland 1984; Kasting et al. 1992, 1993a). After being outgassed, these molecules are dissociated by photolysis due to the high EUV flux of the young Sun and by reactions with $\mathrm{OH}$ and $\mathrm{O}$ radicals produced from the photolysis of $\mathrm{H}_{2} \mathrm{O}$ and $\mathrm{CO}_{2}$ molecules (e.g. Holland 1962; Kuhn and Atreya 1979; Kasting 1982, 1993a, 1998; Zahnle et al. 2010; Wordsworth 2016a). Furthermore, at the high temperatures that characterize planetary surfaces during magma ocean phases, atmospheric $\mathrm{NH}_{3}$ and $\mathrm{N}_{2}$ undergo fast photochemical and thermochemical reactions which can lead to direct dissolution of reduced nitrogen species $\left(\mathrm{N}_{2} \mathrm{X}\right)$ into the surface. As shown by Wordsworth (2016a), hot magmatic surface conditions on early protoplanetary bodies and planets allow rapid abiotic fixation of atmospheric nitrogen.

Although the initial volatile inventory of protoplanets is difficult to constrain, theoretical studies indicate that the solidification of magma oceans can lead to the formation of thick atmospheres in excess of hundreds of bars of $\mathrm{H}_{2} \mathrm{O}$ and $\mathrm{CO}_{2}$ (e.g. Elkins-Tanton 2008; Lebrun et al. 2013; Hamano et al. 2013; Massol et al. 2016; Salvador et al. 2017). Table 2 shows possible parameters of the steam atmospheres generated by the outgassing of putative magma oceans of different depths on early Venus (Lichtenegger et al. 2016), Earth (Elkins-Tanton 2008) and Mars (Erkaev et al. 2014; Odert 2016) along with estimates of the time needed for the outgassed water vapour to condense.

The presence and nature of the outgassed atmosphere controls the lifetime of a magma ocean, since the released greenhouse volatiles exert a blanketing effect that can significantly delay solidification (e.g. Abe and Matsui 1985; ZaHnle et al. 1988; Abe 1997; Elkins-Tanton 
Table 2 The table shows examples of partial pressures of $\mathrm{H}_{2} \mathrm{O}\left(P_{\mathrm{H}_{2} \mathrm{O}}\right)$ and $\mathrm{CO}_{2}\left(P_{\mathrm{CO}_{2}}\right)$ in the outgassed atmosphere after the solidification of the magma ocean is completed. For magma oceans of different depths on Venus, Earth and Mars, and various initial concentrations of $\mathrm{H}_{2} \mathrm{O}\left(X_{\mathrm{H}_{2} \mathrm{O}}\right)$ and $\mathrm{CO}_{2}\left(X_{\mathrm{CO}_{2}}\right)$, The last column $\left(t_{c}\right)$ indicates an estimate of the time after which the water vapour in the atmosphere is expected to condense and form a liquid ocean, if one neglects shock degassing of surface materials (i.e., volatiles, silicates) caused due to frequent impactors and related blanketing effects of the atmosphere after the magma ocean solidified (e.g., Abe and Matsui 1985) (after Elkins-Tanton 2008*; Erkaev et al. 2014 ${ }^{\dagger}$; Lichtenegger et al. $2016^{\ddagger}$ ).

\begin{tabular}{l|lllll}
\hline Magma ocean & $X_{\mathrm{H}_{2} \mathrm{O}}[\mathrm{wt} \%]$ & $X_{\mathrm{CO}_{2}}[\mathrm{wt} \%]$ & $P_{\mathrm{H}_{2} \mathrm{O}}[\mathrm{bar}]$ & $\mathrm{P}_{\mathrm{CO}_{2}}[\mathrm{bar}]$ & $t_{c}[\mathrm{Myr}]$ \\
\hline $\begin{array}{l}\text { Venus } \\
\text { 2000 km deep }\end{array}$ & 0.1 & 0.02 & 450 & 100 & $\geq 10.0 \rightarrow \infty$ \\
$\begin{array}{l}\text { Earth } \\
2000 \mathrm{~km} \text { deep }\end{array}$ & 0.05 & 0.01 & 200 & 40 & $\approx 1.0$ \\
$\begin{array}{l}\text { Mars } \\
500 \mathrm{~km} \text { deep }\end{array}$ & 0.1 & 0.02 & 50 & 10 & $\approx 0.1$ \\
& 0.2 & 0.04 & 110 & 22 & $\approx 0.1$ \\
\hline
\end{tabular}

2008; Lebrun et al 2013; Hamano et al. 2013; Massol et al. 2016; Salvador et al. 2017). A global terrestrial magma ocean that simply cools by directly radiating heat into space would solidify completely in a few thousand years (Monteux et al. 2016). For bodies that solidify under a thick steam atmosphere, the situation can be very different depending on their distance from the Sun. While water vapor outgassed from a magma ocean would condense in $\approx 0.1 \mathrm{Myr}$ at $1.5 \mathrm{AU}$ on Mars, it would do so in $\approx 1 \mathrm{Myr}$ at $1 \mathrm{AU}$ on Earth and in $\approx 10 \mathrm{Myr}$ or even longer at $0.7 \mathrm{AU}$ on Venus whose orbit is too close to the Sun for the steam atmosphere to reach condensation conditions (e.g. Hamano et al. 2013; Lebrun et al. 2013; Massol et al. 2016; Salvador et al. 2017). Indeed, Venus' orbital distance is close to a critical value, below which a magma ocean can be sustained for as long as $100 \mathrm{Myr}$, a time during which the planet would slowly desiccate via hydrodynamic escape (Hamano et al. 2013). On the contrary, beyond such a critical distance, water condensation can be achieved. The first water oceans may have thus formed on early Earth and Mars after condensation of water degassed from their magma oceans (Hamano et al. 2013; Lebrun et al. 2013; Massol et al. 2016; Salvador et al. 2017). Particularly for the Earth, this hypothesis agrees with oxygen-isotope evidence from ancient zircons indicating the presence of liquid water on the Earth's surface as early as 4.3-4.4 Gyr ago (Mojzsis et al. 2001; Wilde et al. 2001).

The Sm-Nd systematics of the Martian SNC meteorites ${ }^{1}$ requires at least a shallow longlived magma ocean on Mars with a lifetime in excess of $\approx 100 \mathrm{Myr}$ (Debaille et al. 2007). This timescale, however, is significantly longer than the solidification time of the order of $\approx 0.1$ Myr inferred from models of magma ocean cooling and outgassing (Lebrun et al. 2013). This discrepancy can be probably solved by noticing that during the first $100 \mathrm{Myr}$ of the Solar System protoplanets have been frequently hit by impactors (e.g., Abe and Matsui 1985, 1988; Genda and Abe 2005; Maindl et al. 2015; Brasser et al. 2016). A continuous flux of impactors may have kept the Martian surface and shallow mantle largely or fully molten for a significant period of time (Maindl et al. 2015), possibly explaining a prolonged magma ocean phase as required by the isotopic evidence of the SNC meteorites. This hypothesis is also in agreement with Abe and Matsui (1985) who showed that impacts can form a silicate/dusty atmosphere by shock degassing, which keeps the surface temperature of accreting proto-planets in magma. According to these researchers not only early Mars but also the accreting proto-Earth was

\footnotetext{
1 Martian SNC meteorites are named after the three representative members Shergotty, Nakhla, Chassigny of a group of 33 achondrite meteorites.
} 
most likely covered by a global magma ocean, at least until the Moon-forming giant impact event.

In general, the timescale of solidification of magma oceans is relatively short compared to the period over which large-scale collisions are likely to occur - at least for bodies orbiting beyond a certain critical distance from the Sun like the Earth and Mars (Hamano et al. 2013). Therefore, it appears likely for accreting bodies to experience catastrophic multiple magma ocean phases during their early history. Indeed at least two episodes of magma ocean outgassing have been suggested for the Earth in order to explain the large differences in the isotopic ratio of ${ }^{3} \mathrm{He} /{ }^{22} \mathrm{Ne}$ between the depleted mantle and a primitive deep reservoir (Tucker and Mukhopadhyay 2014).

\subsection{Volatile loss from planetary embryos}

As pointed out above and illustrated in Fig. 3 large planetesimals accrete within about $10^{5}$ years to bodies with sizes of tens to hundreds of kilometers. Then, these large planetesimals collide and form planetary embryos. During this formation process silicate and metallic materials of planetary embryos formed magma ponds or global magma oceans via impacts, gravitational energy or short lived radioactive isotopes (e.g. Albaréde and Blichert-Toft 2007; Elkins-Tanton 2008, 2012). In such cases planetary embryos would also episodically catastrophically outgas fractions of their initial volatiles, resulting in dryer and volatile poorer building blocks of terrestrial planets with age.

After the magma ocean related outgassing, $\mathrm{H}_{2} \mathrm{O}$ molecules in the upper region of the hot steam atmosphere will be dissociated. Because of the dissociation, $\mathrm{H}_{2}$ molecules and $\mathrm{H}$ atoms will dominate the upper atmosphere. Such an atmosphere would then expand hydrodynamically and $\mathrm{H}$ atoms will escape efficiently as a result (e.g., Watson et al. 1981; Chassefiére 1996a, 1996b; Tian et al. 2005; Lammer et al. 2013, 2014; Erkaev et al. 2013, 2014; Odert et al. 2018). Odert et al. (2018) applied their hydrodynamical upper atmosphere absorption model also to Mars-mass planetary embryos at orbit locations between 0.7-1.5 $\mathrm{AU}$, and studied the loss of their magma ocean related outgassed steam atmospheres.

Fig. 5 shows the evolution of the steam atmosphere partial pressures with initial values of 85 bar $\mathrm{H}_{2} \mathrm{O}$ and 11 bar $\mathrm{CO}_{2}$ from a Mars-mass planetary embryo at Venus' and Earth's orbits at $0.7 \mathrm{AU}$ and 1.0 AU, respectively (Odert et al. 2018). One can see in Fig. 5 that large planetary embryos would lose their outgassed steam atmosphere at Earth's orbit within $\approx 15.5 \mathrm{Myr}$ and at $\approx 12.5 \mathrm{Myr}$ within Venus' orbit, if the young Sun were a weakly active, slow rotating G-star. If the EUV flux were higher, the steam atmosphere would have been lost even faster. Less massive steam atmospheres would also have been lost much faster and lower mass planetary embryos would never build up a dense steam atmosphere, since their outgassed volatiles are lost quickly via thermal escape of the so-called "boil-off" process (e.g. Owen and Wu 2016; Fossati et al. 2016; Odert et al. 2018). One can also see that for the 1.0 AU case, the condensation point of $\mathrm{H}_{2} \mathrm{O}$ would be reached after about $1.0 \mathrm{Myr}$ (Hamano et al. 2013; Lebrun et al. 2013; Massol et al. 2016), if one neglects continuous impacts by smaller planetesimals and planetary embryos. Under such environmental conditions the loss rates may be lower but the atmosphere would also be lost after several tens of Myr. Note that during this efficient escape phase, nitrogen and noble gases such as $\mathrm{Ar}$, Ne, $\mathrm{Kr}$, Xe would also be lost from large planetary embryos. Thus, one can conclude that volatile-rich planetary embryos that experience magma ocean scenarios in the inner Solar System become dryer and less volatile-rich, while they are growing to larger protoplanetary bodies. From the results of Genda and Abe $(2003,2005)$ and Odert et al. (2018) one 

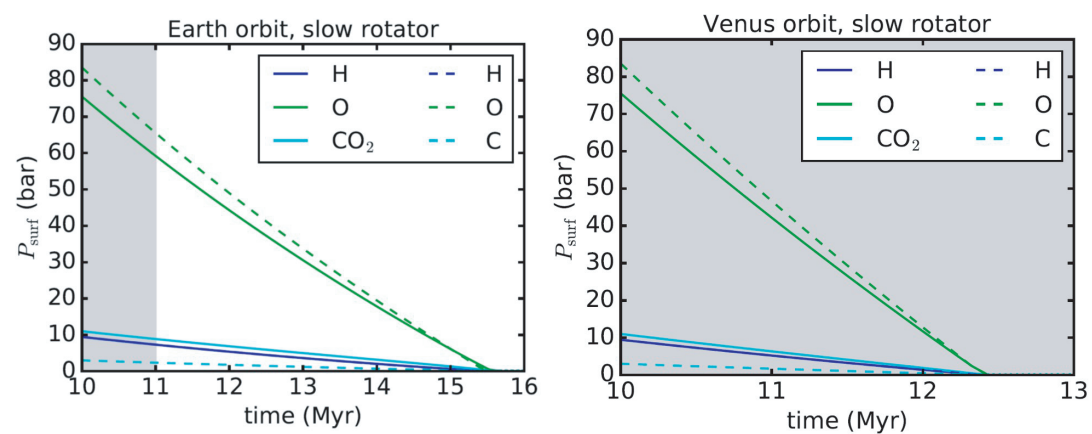

Fig. 5 Evolution of the partial surface pressures of escaping steam atmospheres $\left(\mathrm{H}_{2} \mathrm{O}: 85\right.$ bar, $\mathrm{CO}_{2}: 11$ bar $)$ on a Mars-mass planetary embryo at 1.0 AU (left panel) and 0.7 (right panel), exposed by the enhanced EUV flux of a low active slow rotating young Sun. Solid lines correspond to cases where the $\mathrm{CO}_{2} \mathrm{molecule}_{\text {is not }}$ dissociated and the dashed lines correspond to cases where $\mathrm{CO}_{2}$ molecules have been dissociated. According to Lebrun et al. (2013) the shaded area marks the condensation time of steam atmospheres to form planetary oceans (courtesy of P. Odert).

can conclude that the volatile inventory (i.e., water, carbon and nitrogen related) of large planetary embryos will be modified during and after the time when they collide and accrete to larger protoplanetary cores. One should note that these results are in agreement with Marty (2012) that unfractionated smaller planetesimals (i.e. $\approx 2 \pm 1 \%$ carbonaceous chondrites) are probably better suited for the delivery of volatile elements, and Mg isotope evidence that accretional vapour loss shapes planetary compositions (Hin et al. 2017). The latter study investigated isotopic fractionation processes observed on differentiated planetary bodies that are chemically fractionated compared to primitive, chondritic meteorites and, by inference, the primordial disk from which they formed. Hin et al. (2017) suggest that isotopic fractionation between liquid and vapour, followed by vapour outgassing and escape during accretionary growth of planetesimals modifies their compositions. The model results of Hin et al. (2017) indicate that the isotopic compositions of $\mathrm{Mg}$, Si and $\mathrm{Fe}$, and the relative abundances of the major elements of Earth and other planetary bodies, are a natural consequence of $\approx \mathbf{4 0} \%$ by mass vapour loss from growing planetesimals.

In agreement with planetary dynamics model results related to the "Grand Tack" hypothesis (Walsh et al. 2011; O'Brien et al. 2014) and the recent Ru isotope analysis of primitive meteoritic material (Fischer-Gödde and Kleine 2017), it is now expected that volatile-rich carbonaceous chondritic bodies were scattered from the outer into the inner Solar System early in Earth's, Venus' and Mars' accretion phase. In such a case one can expect that these bodies originated in a very cold environment beyond the ice-line, where the EUV flux of the young Sun was low. Therefore, magma ocean related outgassed volatiles of large planetesimals and embryos in the outer Solar System experienced low escape rates and remained most likely volatile-rich.

When the inward and outward migration of Jupiter in the "Grand Tack" scenario scattered volatile-rich primitive planetesimals to the inner planets (Walsh et al. 2011; O'Brien et al. 2014), one can expect that several large volatile-rich carbonaceous chondritic impactors hit more or less directly growing early terrestrial proto-planets. In such a case most volatiles, including a few Earth oceans, could have been delivered to the accreting planets via direct collisions. 
3.5 Noble gas fractionation in early atmospheres

As discussed above, the earliest atmospheric evolution of larger terrestrial planets is strongly connected to the protoplanetary nebular and the lifetime of the gas disk. This is directly related to the way how nonradiogenic $\mathrm{Ne}, \mathrm{Ar}, \mathrm{Kr}$, and $\mathrm{Xe}$ isotopes, and other volatile elements (i.e., $\mathrm{K}, \mathrm{Na}$, etc.) have been fractionated. A nebular-based $\mathrm{H}_{2}$-envelope would also explain why Earth's environment contains solar-like primordial ${ }^{3} \mathrm{He}$ (e.g., Clarke et al. 1969; Mamyrin et al. 1969; Craig and Lupton 1976). Moreover, solar-like $\mathrm{Ne}$ with a solar $\mathrm{He} / \mathrm{Ne}$ ratio was discovered in some plumes (Dixon et al. 2000; Porcelli et al. 2001; Yokochi and Marty 2004; Marty and Yokochi 2006) as well as hints of a solar Ar component in basalts (e.g., Honda et al. 1991; Moreia et al. 2001). According to Caffee et al. (1988, 1999) a solar component of Xe can also be found in gases emitted from wells. On the other hand according to Feigelson et al. (2002) and Halliday et al. (2014, and references therein) noble gases could have also been incorporated into early formed planetesimals by the intense solar wind of the young Sun after the gas disk evaporated.

Fig. 6 illustrates how the hydrodynamic escape of $\mathrm{H}$ atoms can drag noble gases and fractionate their isotopes in time. As long as the gas disk is present planetary building blocks cannot be implanted by solar wind particles, including solar wind related noble gas isotopes. After the nebular gas evaporated it is expected that the early solar wind density was up to about 50 times denser and about 2-3 times faster than today (e.g., Johnstone et al. 2015b, 2015c). Geiss (1973) discovered that the isotopic composition of the solar noble gases in some meteorites is very similar to that in the present solar wind while others, including certain surface areas on the Moon may contain traces of the early solar wind (Wieler and Heber 2003, Wieler et al. 2016). Therefore, after the protoplanetary nebula disappeared, the remaining planetesimals and small planetary embryos delivered noble gases, that have been trapped inside them via solar wind interaction with these airless bodies (Feigelson et al. 2002; Halliday 2014, and references therein).

\subsubsection{Early Venus and Earth}

According to Cameron (1983) and Pepin (1991, 1997) Venus' high noble gas abundance taking into account all the uncertainties indicates that the heavier nonradiogenic noble gases in the atmosphere are not greatly different from their primordial stages. Odert et al. (2018) applied a hydrodynamical upper atmosphere model to proto-Venus atmospheric evolution scenarios. These authors found that hydrodynamic escape modeling can reproduce the present day observed Venus ${ }^{20} \mathrm{Ne} /{ }^{22} \mathrm{Ne}$ and ${ }^{36} \mathrm{Ar} /{ }^{38} \mathrm{Ar}$ isotope ratios if proto-Venus grows quickly to a proto-core with masses $>0.6 M_{\text {Earth }}$ inside the gas disk, so that a thin $\mathrm{H}_{2}$-envelope with solar-like noble gas isotopes embedded could be accumulated. These authors investigated the dragging of heavier dissociation products like $\mathrm{O}, \mathrm{C}, \mathrm{O}_{2}, \mathrm{CO}, \mathrm{CO}_{2}$ and noble gases such as Ne and Ar. The adopted equations, which have been derived by Zahnle et al. (1986) and Zahnle et al. (1990), are applicable to atmospheres with one or two major species and an arbitrary amount of additional minor species.

Morevover, the results of Odert et al. (2018) indicate that a young Sun with an EUV activity close or similar to that of a slow rotator as shown in Fig. 1, would be the most adequate to reproduce the observed atmospheric noble gas isotope fractionation. At higher EUV, Venus' initial atmospheric $\mathrm{CO}_{2}$ inventory could have been also dragged and lost to space (Odert et al. 2018). The left panel of Fig. 7 shows a possible early Venus combined $\mathrm{H}_{2}$-envelope mixed with an outgassed steam atmosphere scenario that can reproduce the observed $\mathrm{Ne}$ and Ar isotope ratios using the escape models applied in Odert et al. (2018). 


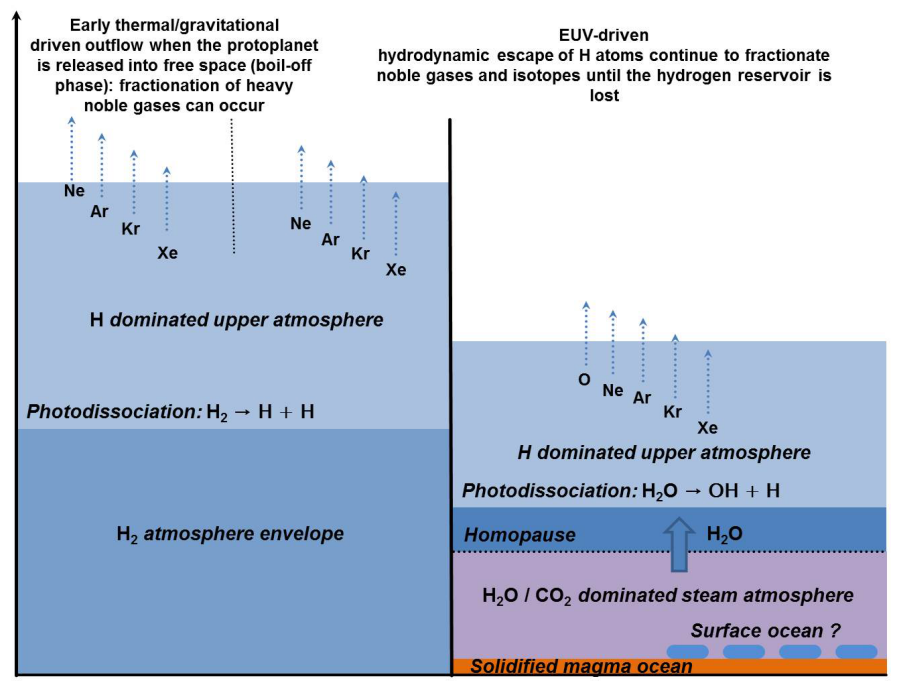

Fig. 6 Illustration of scenarios for hydrodynamic escape of $\mathrm{H}$ atoms that can drag noble gas isotopes from an extended gas disk-based $\mathrm{H}_{2}$-envelope (left), and a less extended magma ocean related outgassed steam atmosphere (right). In a steam atmosphere (right) the $\mathrm{H}_{2} \mathrm{O}$ molecules are dissociated in the upper atmosphere and atomic hydrogen is also lost from the planet and can drag heavier species. Depending on the mass of a planetary embryo or protoplanet, these steam atmospheres are less extended and the loss rates are lower compared to nebular captured $\mathrm{H}_{2}$-envelopes.
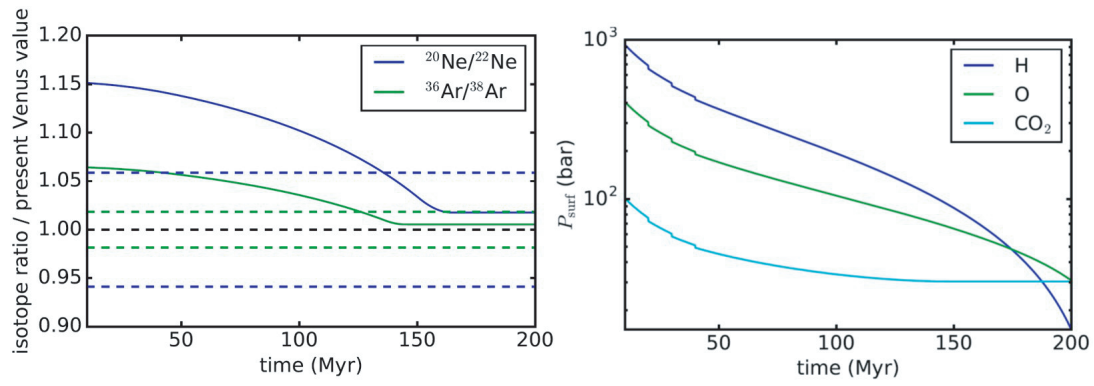

Fig. 7 Example for the evolution of a mixed accumulated $\mathrm{H}_{2}$-envelope captured around a proto-Venus with $\approx 70 \%$ of its mass before the nebular evaporated and a steam atmosphere $\left(450\right.$ bar $\mathrm{H}_{2} \mathrm{O}, 100$ bar $\left.\mathrm{CO}_{2}\right)$, including later finished accretion by impacts with large planetary embryos and a slow rotating young Sun (Odert et al. 2018). Left figure shows the corresponding Ne and Ar isotope fractionation that can be reproduced under these assumptions during about $150 \mathrm{Myr}$ with a slow rotating weakly EUV active young Sun. The dashed lines show the ranges of the presently measured atmospheric Venusian isotope ratios. Right figure shows the atmospheric partial surface pressure evolution (courtesy of P. Odert).

The assumed protoplanet scenario, which can reproduce the observed atmospheric ${ }^{20} \mathrm{Ne} /{ }^{22} \mathrm{Ne}$ and ${ }^{36} \mathrm{Ar} /{ }^{38} \mathrm{Ar}$ isotope ratios, is also in agreement with the "Grand Tack" hypothesis (Walsh et al. 2011; O'Brien et al. 2014). However, if one assumes that early Venus began its accretion after the gas disk disappeared, the results of Odert et al. (2018) indicate that it is not possible to reproduce the observed noble gas isotope ratios with realistic atmosphere scenarios, i.e. without losing a 100 bar $\mathrm{CO}_{2}$ atmosphere to space and leaving an unrealistical large amount of oxygen behind. Therefore, from these recent studies one can conclude in agreement with 


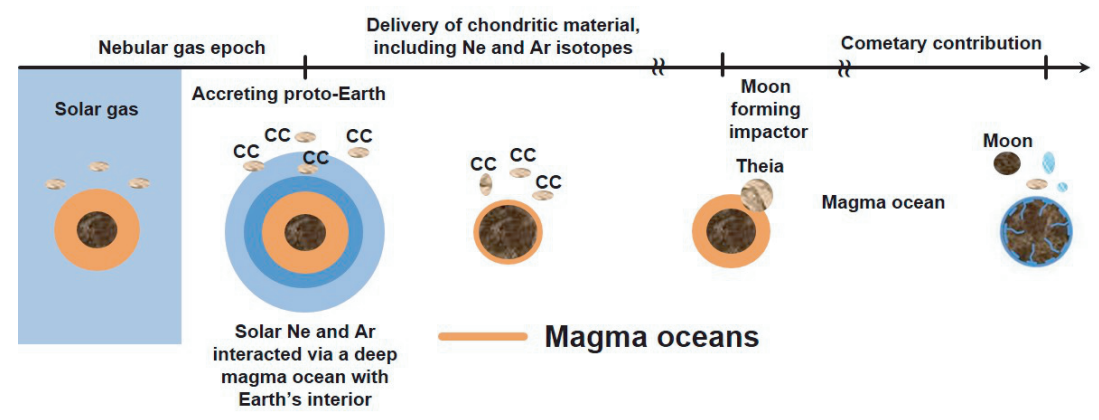

Fig. 8 Illustration of the evolution of early Earth according to observed and analyzed noble gas (i.e., Ar, Ne, etc.) data on Earth. After the loss of a thin nebular-based $\mathrm{H}_{2}$-envelope, impacts of carbonaceous chondrites (CC) accreted the final mass and delivered volatile and noble gases which resulted in a nebular-CC mixture of atmospheric $\mathrm{Ar}$ and $\mathrm{Ne}$ isotopes (after private communications with Bernard Marty).

the "Grand Tack" hypothesis (Walsh et al. 2011; O'Brien et al. 2014) that early Venus most likely grew to a mass inside the nebular large enough to capture a thin $\mathrm{H}_{2}$-envelope, that was afterwards lost via EUV-driven hydrodynamic escape.

While atmospheric $\mathrm{Ne}$ and Ar ratios on Venus are closer to the initial solar rates, on Earth the atmospheric isotope ratios are different and represent solar rates that are modified by a contribution from carbonaceous chondritic material to a dry proto-Earth (e.g., Marty 2012; Bouvier and Boyet 2016). These remnants of the proto-solar nebula are still present in the mantle, indicating a sequestration of nebular gas at an early stage of planetary growth. This is confirmed by the discovery of slightly fractionated solar ${ }^{20} \mathrm{Ne} /{ }^{22} \mathrm{Ne}$ ratios trapped in fluid inclusions from plume-related rocks from Iceland (e.g., Dixon et al. 2000; Porcelli et al. 2001) and the Kola Peninsula, Russia (Yokochi and Marty 2004; Marty and Yokochi 2006). These findings are also in agreement with the hypothesis of Pepin $(1991,1997,2000)$ that some noble gas fraction, which has been found on Earth, such as the so-called U-Xe composition (Pepin 2000), should have been integrated into the planet's interiors via an early $\mathrm{H}_{2}$-envelope getting into contact with a magma ocean. Xe isotopes in Earth's atmosphere are enriched in heavier isotopes. In the case of Venus, however, the $\mathrm{Kr}$ and $\mathrm{Xe}$ isotopic ratios are currently unknown, i.e. the measurement of these ratios would be a very important scientific driver for future Venus space missions, like the planned Russian Venera-D spacecraft.

Fig. 8 illustrates the most likely evolution scenario of proto-Earth. First, the isotopically solar-like noble gases experienced hydrodynamic atmospheric escape of a thin nebular-based $\mathrm{H}_{2}$-envelope (e.g. Pepin 1991, 1997; Becker et al. 2003), which should have been lost earlier compared to Venus. Carbonaceous chondrites (CC) then added noble gas isotopes with CC fractionation to the terrestrial reservoir. Then, after solidification of the magma ocean, the present day observed $\mathrm{Ar}$ and $\mathrm{Ne}$ isotope ratios thus evolved by outgassing of a mixture of solar nebula and chondritic noble gas ratios (Marty and Allé 1994).

\subsubsection{Early Mars}

On Mars the isotopic components of atmospheric $\mathrm{Kr}$ are close to those of the solar wind and remained mainly unfractionated (e.g., Pepin 1991; 2000; Becker et al. 2003; Conrad et al. 2016). However, observational evidence from atmospheric measurements of present Mars indicates that during early periods, atmospheric escape of isotopes (i.e. ${ }^{36} \mathrm{Ar},{ }^{38} \mathrm{Ar},{ }^{20} \mathrm{Ne}$, 
${ }^{22} \mathrm{Ne},{ }^{124-136} \mathrm{Xe}$ etc. and its related fractionation, should have occurred (Jakosky et al. 2017; Cassata 2017).

One should also note that according to Marty and Meibom (2007), Earth's atmospheric noble gas abundance was most likely also modified by cometary impactors from the Kuiperbelt during the late heavy bombardment $\approx 3.8-4.1$ Gyr ago. Therefore, degassing of cometary material on its journey towards the inner Solar System should also be considered. Tian et al. (2009), Erkaev et al. (2014) and Odert et al. (2018) found that Mars could have lost a fraction of its outgassed volatiles by EUV-driven hydrodynamic escape. If one assumes that early Mars outgassed $\approx 85$ bar $\mathrm{H}_{2} \mathrm{O}$ and $\approx 10$ bar $\mathrm{CO}_{2}$ associated with a solidifying magma ocean, then, according to Odert et al. (2018), the resulting degassed atmospheric water inventory would mainly be lost after $\approx 25 \mathrm{Myr}$ if the young Sun were a slow rotator. If the early Sun were more active and rotated at least moderate quickly, then the whole atmosphere would have been lost during $\approx 18 \mathrm{Myr}$, including molecular $\mathrm{CO}_{2}$ (Erkaev et al. 2014; Odert et al. 2018).

Due to these efficient hydrogen escape rates, noble gases could have also been dragged by the escaping $\mathrm{H}$ atoms. This scenario is in agreement with the early atmosphere evolution hypothesis of Pepin (1994, 2002). Recently Jakosky et al. (2017) and Kurokawa et al. (2017) estimated the amount of atmosphere lost to space through time by using measurements of the upper-atmospheric structure made by the MAVEN spacecraft. Based on variations in ${ }^{38} \mathrm{Ar} /{ }^{36} \mathrm{Ar}$ ratios between the homopause and exobase levels, both studies came to the conclusion that Mars' surface pressure was most likely $\geq 0.5$ bar $\approx 4$ Gyr ago.

That Mars lost most of its initial atmosphere and was much drier than Earth $\approx 4-4.3$ Gyr ago is also in agreement with Xe data inferred from two ancient Martian meteorites, ALH 84001 and NWA 7034 (Cassata 2017). The data analysis indicate an early episode of efficient atmospheric escape so that mass fraction of Xe isotopes culminated early in the planet's formation, and little change to the atmospheric Xe isotopic composition has occurred since this time.

However, Mars is more complex because of its small gravity and its lack of an intrinsic magnetic dynamo over the past 4 Gyr. It was shown that nonthermal atmospheric escape processes such as atmospheric sputtering can also fractionate isotopes (e.g., Jakosky et al. 1994; Hutchins and Jakosky 1996; Artreya et al. 2013; Jakosky et al. 2017; Kurokawa et al. 2017).

3.6 Most likely evolution scenarios up to the "late veneer"

The most plausible formation scenarios for early Venus, Earth and Mars during the first 100 Myr, after the Solar System originated $\approx 4.6$ Gyr ago, are illustrated in Fig. 9. The evolution of the three planets during this initial time period defines the boundary conditions for the next steps of their evolution. As discussed above the latest findings related to isotope data and in the dynamical evolution and formation of the Solar System terrestrial planets indicate that proto-Venus and proto-Earth grew to $\approx \mathbf{0 . 5}-0.75 M_{\text {Earth }}$ within the first $10 \mathrm{Myr}$, so that a thin $\mathrm{H}_{2}$-envelope from the disk could have been captured. Noble gases from the solar nebula could be incorporated via magma oceans into the planetary interiors. During the loss of these $\mathrm{H}_{2}$ envelopes, non-radiogenic noble gases have been fractionated via hydrodynamic escape of hydrogen (Odert et al. 2018).

At Earth's orbit, water, which has been catastrophically outgassed after the final deep magma ocean solidified, could condense after $\approx 1.0$ - 1.5 Myr (Lebrun et al. 2013; Sleep et al. 2017) when the impact rate of large impactors become low (i.e., after the Moon forming 
giant impact event). The first stable liquid water ocean was produced on the surface. As illustrated in Fig. 9, a large fraction of the accreted water, including water that arrived from outer Solar System bodies during the main stages of Earth's formation, could also exist in liquid form so that huge amounts of water most likely entered during or after the Moon-forming event into early Earth's mantle. This is also consistent with $\mathrm{He} / \mathrm{Ne}$ isotopic data, suggesting that the bulk amount of Earth's volatiles was delivered to Earth before the Moon-forming impact (Tucker and Mukhopadhyay 2014). Following Hier-Majumder and Hirschmann (2017), a large amount of volatiles (in their study up to $77 \% \mathrm{H}_{2} \mathrm{O}$ and $12 \% \mathrm{CO}_{2}$ ) could be incorporated into the mantle during solidification of the magma ocean. This water influenced the thermal evolution and rock mechanics in the mantle during its later evolution. Water may also play an important role for the later initiation of plate tectonics (e.g., Hopkins et al. 2008; Shirey et al. 2008; Korenaga 2013), even though the starting point of (proto-)plate tectonics on Earth is strongly debated (see Sect. 4.1). That present-day Earth has more water in its interior than in the surface ocean is in the meantime supported by the recent finding of a hydrous mantle transition zone (Karato, 2011; Pearson et al. 2014; Schmandt et al. 2014; Plümper et al. 2017) at depths between 410 and 660 kilometres. This water reservoir is replenished by subduction of water into the mantle (Parai and Mukhopadhyay 2012; Höning et al. 2014). Water, especially in the crust (Jackson 2002), as well as in the upper mantle play an important role for the development of modern plate tectonics and related terrestrial magmatism, mechanisms which seem to have failed at least for Mars. Venus' past is hidden from us, since the entire crust has been recently resurfaced, but could have been similar to today during its entire evolution.

That the terrestrial planets obtained most of their nitrogen inventory most likely also during the main stage of terrestrial planet formation is backed from isotopic measurements (Marty 2012; Füri and Marty 2015). Present Earth's atmospheric ${ }^{14} \mathrm{~N} /{ }^{15} \mathrm{~N}$ ratio is near to the values discovered in ordinary and carbonaceous chondrites (Marty 2012). On the other hand, while the solar and gas giant nitrogen reservoirs are much lighter (Owen et al. 2001), $\mathrm{Ru}$ isotope anomalies in late veneer impactors exclude a nitrogen origin from those impacts (Fischer-Gödde and Kleine 2017). From these findings one can expect that most of Earth's and most likely also Venus' and Mars' $\mathrm{N}_{2}$ was delivered by bodies early during the main stage of planet accretion with a composition close to carbonaceous chondrites. According to Wordsworth (2016a) it is reasonable that the majority of the nitrogen was delivered by volatile-rich impactors of chondritic composition and was in the form of $\mathrm{NH}_{3}$-ices or organic compounds like HCN. As pointed out above, one can expect that atmospheric nitrogen should have been depleted very fast into the surface by direct dissolution as long as it was magmatic or hot, which was certainly an important nitrogen fixation process on the early terrestrial planets (Wordsworth 2016a). As pointed out above, early Venus accumulated most likely a large fraction of its $\mathrm{CO}_{2}$-inventory in the atmosphere through the outgassing of the magma ocean and impacts of volatile-rich chondritic bodies during the later stages of accretion. If Earth obtained water and other volatiles by impactors from the outer Solar System during its main accretion phase (Walsh et al. 2011; O’Brien et al. 2014; Fischer-Gödde and Kleine 2017), then one can expect that Venus and Mars most likely also obtained their water and other volatiles (e.g., Carr 1989; Manning et al. 2009; Pham et al. 2011; Adock et al. 2016) during the same time period.

As outlined in the previous sections, recent studies, which considered also magma ocean related effects (Marcq 2012; Elkins-Tanton 2012; Lebrun et al. 2013; Hamano et al. 2013; Massol et al. 2016) and nebular captured $\mathrm{H}_{2}$ envelopes (Ikoma and Genda 2006; Pierrehumbert and Gaidos 2011; Massol et al. 2016; Stökl 2015 2016; Odert et al. 2018) yield climate conditions on early Venus that would be too hot for water to condense, unless 


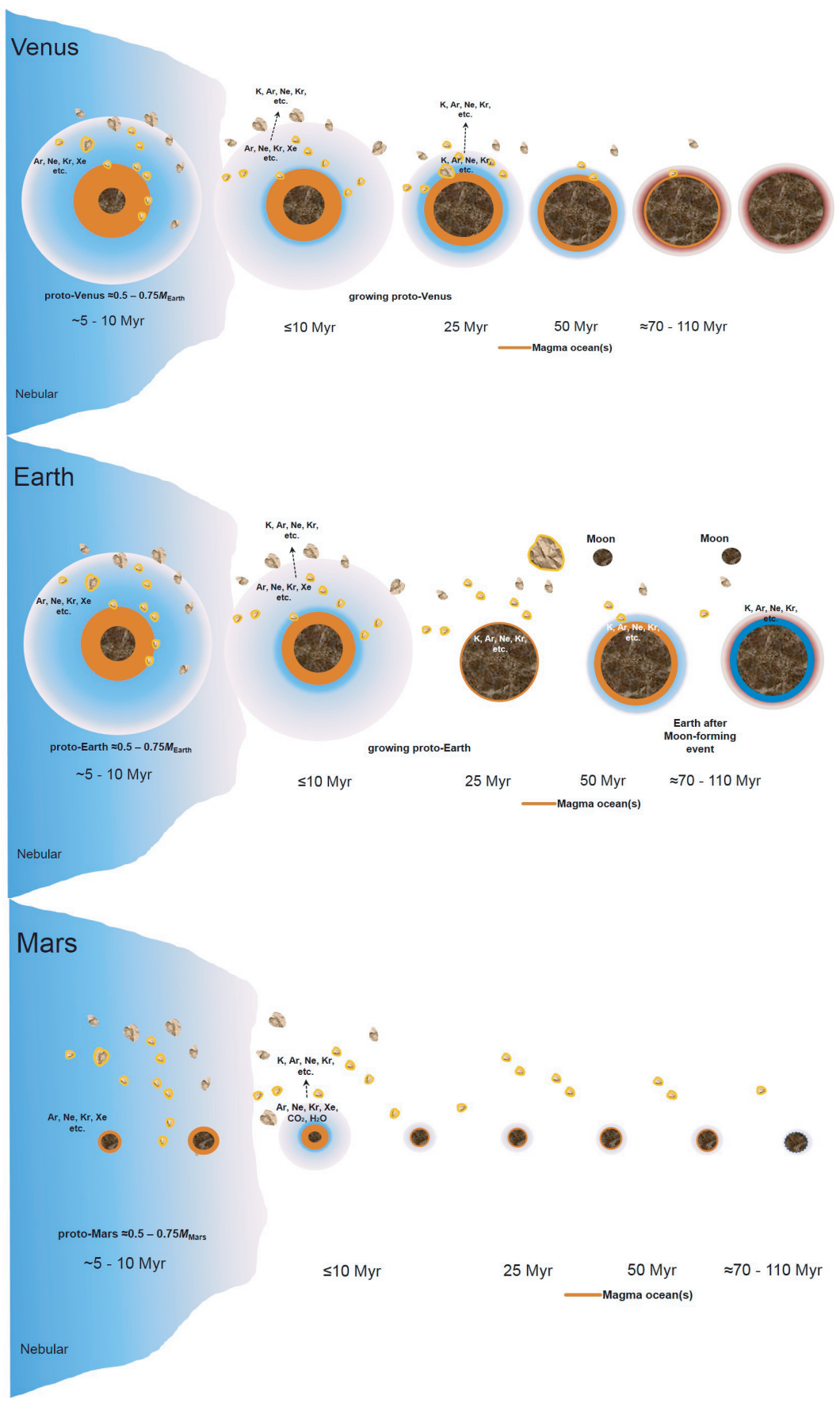

Fig. 9 Illustration of the most likely formation scenarios of early Venus, Earth and Mars from the origin of the Solar System $\approx 4.567 \mathrm{Gyr}$ ago during the first $100 \mathrm{Myr}$. Most likely proto-Venus and proto-Earth grow to about $0.5-0.75 M_{\text {Earth }}$ of their initial masses before the gas disk evaporated. During this phase solar component related noble gases could have been integrated into the planetary interiors via the interaction with magma oceans below the $\mathrm{H}_{2}$-envelopes, while the much smaller accreting Mars could not capture $\mathrm{H}_{2}$ from the nebular. After the nebular gas disappeared solar wind related noble gases that have been trapped in planetesimals, which continued to bombard proto-Mars have been delivered to the planet's magmatic crust/mantle. 
a shielding cloud layer would have formed, keeping the surface temperatures on early Venus cool enough for liquid water at the surface (Kasting and Pollack 1983; Kasting et al. 1984b; Kasting 1988; Way et al. 2016; Salvador et al. 2017). The consequences related to the atmospheric evolution of early Venus in case the planet had a liquid water ocean on its surface is discussed in the following Section. If water never existed in liquid form at the surface and could not be recycled into the mantle, the interior of Venus would have remained much dryer compared to the mantle of early Earth. On the other hand, it is unclear, whether a modern form of plate tectonics ever existed on Venus (e.g. O'Neill et al. 2007; Noack et al. 2012).

Due to its low gravity the evolution of Mars' early atmosphere was more sensitive to the EUV flux of the young active Sun compared with Venus and Earth. Depending on the young Sun's EUV activity and related rotation history, Mars could have lost its magma ocean outgassed steam atmosphere early on (Erkaev et al. 2014), or may have kept several bar of $\mathrm{CO}_{2}$ at about $0.1 \mathrm{Gyr}$ (Tian et al. 2009; Amerstorfer et al. 2017), if one assumes that the young Sun was a slow rotator. Depending on impacts and greenhouse gases (i.e., $\mathrm{CH}_{4}, \mathrm{H}_{2}$, etc.) additional to $\mathrm{CO}_{2}$, water may have existed on early Mars in liquid form only episodically during the pre-Noachian. The planet also cooled faster compared to Earth due to its smaller size. The environmental conditions therefore did not favor plate tectonics, at least not over geological time periods comparable to those of Earth (O'Neill et al. 2007).

\section{$4 \mathrm{CO}_{2}$ atmosphere loss to the sub-surface and space}

Although there is a controversy over how and when modern-type plate tectonics started, the main difference between early Venus, Mars and early Earth from a geological point of view was, that Earth could evolve at least a form of crust recycling during a few hundreds of Myrs after the late veneer. Maybe early Mars also developed a short plate tectonics phase in the first few hundreds of Myr (Frey 2006). In the following sections we discuss how this difference dominated the further atmospheric evolution and that it was essential that Earth's atmosphere could evolve to a planet where life originated.

\subsection{Plate tectonics vs. one-plate planets}

Most researchers agree that plate tectonics was not present in the earliest stages of Earth's history. Today's form of plate tectonics may have evolved from some earlier proto-plate tectonics regime such as plume-induced subduction (e.g., van Kranendonk 2010; Gerya et al. 2015; Baes et al. 2016). The nature of metamorphic rocks suggests a hotter Earth in the late Hadean or early Archean where plates could not subduct to the depths required to form rocks such as coesite-bearing eclogites, which may have led to the later initiation of deep subduction (van Kranendonk 2010, and references therein).

Subduction and seafloor spreading are primary drivers of plate motions and the warmer early Earth may have had a weaker, less dense lithosphere and thus mantle convection processes may have been different during those times (e.g., van Kranendonk 2010). Fig. 10 compares plate tectonics (Earth) with stagnant lid convection (Venus, Mars). A large amount of water (Pearson et al. 2014; Schmandt et al. 2014; Plümper et. al 2017), interacted with Earth's mantle environment during the early Hadean, possibly leading to conditions favorable for a proto-plate tectonics regime. Finally, the modern form of plate tectonics started to operate most likely during the Archean (e.g., Nutman et al. 2002; van Kranendonk 


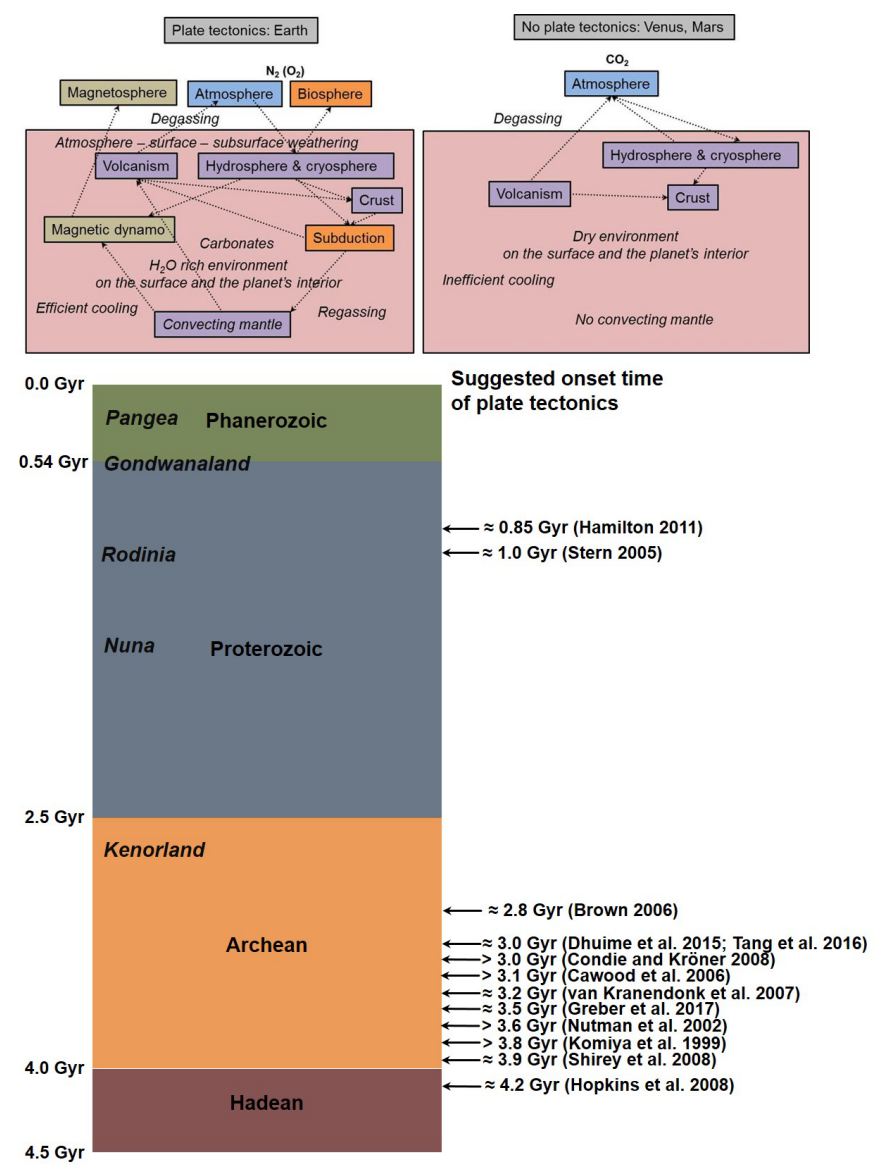

Fig. 10 Top: Illustration of mantle convection of a planet (Earth) where plate tectonics operates (left) compared to planets (Venus, Mars) which have a mantle with stagnant lid convection. The lower panel is a schematic view of the geologic eras and suggestions based on different models and observational constraints for the expected onset time of plate tectonics. Times of Earth's supercontinental historical cycles are also shown.

et al. 2007; Shirey et al. 2008; Condie and Kröner 2008; Dhuime et al. 2015; Tang et al. 2016; Greber et al. 2017) and remained active until present.

If plate tectonics operates, the top thermal boundary layer is continuously recycled back into the mantle. This process to work requires a mechanism compensating for the high, temperature-dependent viscosity of silicate rocks (e.g. Korenaga 2007, 2008, 2013). Water in the lithosphere and crust plays an important role in these processes because it influences the friction within rocks (Korenaga 2013). In the case, that water fills the pore space in rocks, the pore fluid pressure can then reduce the shear strength of the rocks (Turcotte and Schubert 1982; Korenaga 2008, 2013).

Plate bending at subduction zones may fracture the oceanic lithosphere to substantial depths (Ranero et al. 2003; Korenaga 2013). Large water reservoirs in the mantle and on the surface are interacting (Schmandt et al. 2014). Although, the main processes which drive plate tectonics are not fully understood, the minimum requirements are a sufficient mass 
relevant for the heat flow to drive mantle convection, and a certain amount of water in the planet's interior to lubricate plate motion (e.g., Regenauer-Lieb et al. 2001; Solomatov 2004).

Fig. 10 shows also the possible onset times for plate tectonics on early Earth obtained from various research teams, models and observational constraints. The predictions shown in Fig. 10 illustrate that there is a wide diversity of suggestions that have been published over more than a decade, and this list is not exhaustive.

However, one can see that most studies imply that plate tectonics started to operate during the Archean and, from more recent results related to the outgassing and build up of early Earth's $\mathrm{N}_{2}$ atmosphere (Mikhail and Sverjensky 2014), an onset between 3.0 - 4.0 Gyr ago (e.g. Komiya et al. 1999; Nutman et al. 2002; Shirey et al. 2008; Dhuime et al. 2015; Tang et al. 2016; Greber et al. 2017) seems reasonable. Most lithospheric studies agree that at about 3.0 Gyr ago early Earth most likely experienced a stepwise change in tectonic style (van Kranendonk 2010).

\section{$4.2 \mathrm{CO}_{2}$ surface weathering and atmospheric escape}

Atmosphere-ocean-surface interaction processes have been responsible for $\mathrm{CO}_{2}$ weathering from the atmosphere during the Hadean and transformation into carbonates. However one should note that early Earth lacked extensive, stable continental platforms during the Hadean (e.g., Flament and Coltice 2008) on which carbon could be stored in the form of carbonate minerals for geologically significant periods of time. In the absence of continents, the Earth's carbon must have been either in the atmosphere or ocean or in the form of shortlived sedimentary deposits on the ephemeral sea floor (Walker 1985). According to Walker (1985) one can expect that initially the atmospheric $\mathrm{CO}_{2}$ partial surface pressure was high $(\geq 10$ bar), but declined rapidly as a result of the growth of the global sedimentary rock reservoir and possibly also as a result of the return of carbon from the exogenic system to the mantle, and finally due to the growth of continents (e.g., Belousova et al. 2010; Dhuime et al. 2012).

Rainwater containing carbonic acid is able to react with most minerals at a rate depending on their chemical stability. The relevant minerals that make up rocks at Earth's land surface react very slowly. Therefore, large-scale $\mathrm{CO}_{2}$ weathering, which is an important sink for carbon, takes place on a timescale of millions of years.

It is expected that large quantities of atmospheric $\mathrm{CO}_{2}$ ends up in the surface and in the planet's interior via weathering of rocks and reprecipitation of weathering products as carbonate sediments (e.g. limestones). The process begins when $\mathrm{CO}_{2}$ dissolves in droplets of $\mathrm{H}_{2} \mathrm{O}$ in the clouds. As shown in Fig. 11 the resulting solution that reaches the surface and ocean water as rainwater is a form of carbonic acidic as

$$
\mathrm{CO}_{2}+\mathrm{H}_{2} \mathrm{O} \rightarrow \mathrm{H}_{2} \mathrm{CO}_{3} .
$$

Carbonic acid can then dissociate into

$$
\mathrm{H}_{2} \mathrm{CO}_{3} \rightarrow \mathrm{HCO}_{3}^{-}+\mathrm{H}^{+},
$$

producing bicarbonate and $\mathrm{H}^{+}$ions. The concentration of $\mathrm{H}^{+}$ions defines the acidity. Hydrogen ions and water react with most common minerals (silicates and carbonates) and can alter the minerals. The products of weathering are predominantly clays (a group of silicate minerals) and soluble ions such as $\mathrm{Ca}, \mathrm{Fe}, \mathrm{Na}$, and $\mathrm{K}$. Some bicarbonate ions also remain in solution and build a remnant of the carbonic acid that was used to weather the rocky surface. As illustrated in Fig. 11, after some time the carbon will be stored on the seafloor in the 


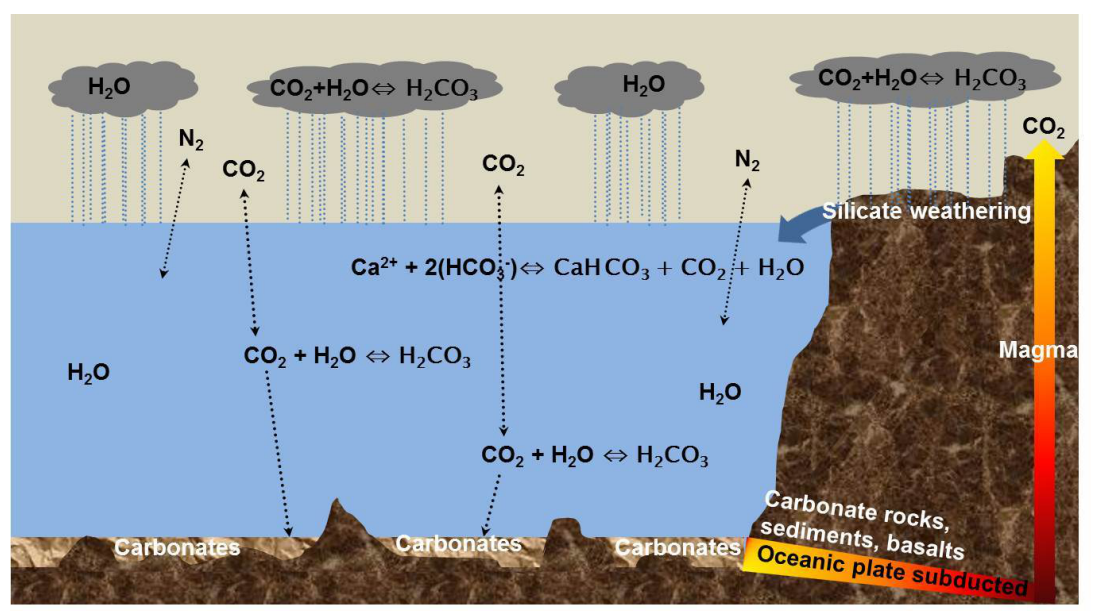

Fig. 11 Illustration of the carbonate-silicate geochemical cycle of atmospheric $\mathrm{CO}_{2}$ weathering and carbonate formation, as well as $\mathrm{CO}_{2}$ outgassing via volcanos.

form of limestone layers. Some of this carbon returns to the atmosphere via metamorphism of limestone at depth in subduction zones or in orogenic belts. Due to the climatological role of $\mathrm{CO}_{2}$, carbon cycle feedbacks acted most likely during the Hadean and maintained Earth's global temperatures fixed within a certain range so that the climate never became too hot or too cold preventing the ocean from evaporation or total freezing. As pointed out in Sect. 2 the young Sun was about 30\% fainter at $\approx 4.3-4.5$ Gyr ago (e.g., Kasting et al. 1984b; Mojzsis et al. 1996; Rosing and Frei 2004; Valley et al. 2002; Güdel 2007; Feulner 2012, and references therein; Charnay et al. 2013; Wolf and Toon 2013; Kunze et al. 2014) and that, as mentioned above, liquid water on the surface of Earth has most likely existed since $\approx 4.3-4.4$ Gyr ago.

For example, when carbonic acid is removed from solution by rock weathering, the reactions will adjust themselves in such a way to produce more carbonic acid. Since the dissolved $\mathrm{CO}_{2}$ is in equilibrium with atmospheric $\mathrm{CO}_{2}$, more $\mathrm{CO}_{2}$ is removed from the atmosphere to replace what was removed from solution by surface weathering (e.g. Sleep 2010). It is expected that this climate feedback response of the $\mathrm{CO}_{2}$ weathering processes played an important role to keep early Earth's average surface temperature above freezing until the late Hadean (e.g. Kasting et al. 1984a, 1993a; Kasting and Schultz 1996; Walker 1985; Kasting and Ono 2006; Haqq-Misra et al. 2008; von Paris et al. 2008; Feulner 2012, and references therein; Charnay et al. 2013; Wolf and Toon 2013; Kunze et al. 2014).

Today, most of the carbon in Earth's interior is present in relatively non-volatile form of carbonates (see Table 1). If one assumes that after the Moon-forming event and its related global magma ocean most of Earth's $\mathrm{CO}_{2}$ inventory was outgassed, than early Earth's

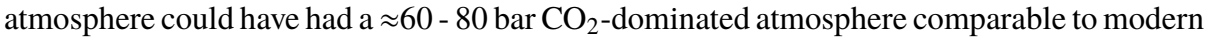
Venus (Ronov and Yaroshevsky 1967; Walker 1985).

Earlier studies by Kasting and Pollack (1983), Kasting et al. (1984), Kasting (1988) and more recent, Way et al. (2016) and Salvador et al. (2017), proposed environmental scenarios, where early Venus could have had also a warm liquid water ocean on its surface. According to Salvador et al. (2017) the end of the rapid cooling stage of the magma ocean is reached when the mantle heat flux becomes negligible compared to the absorbed solar flux. The 


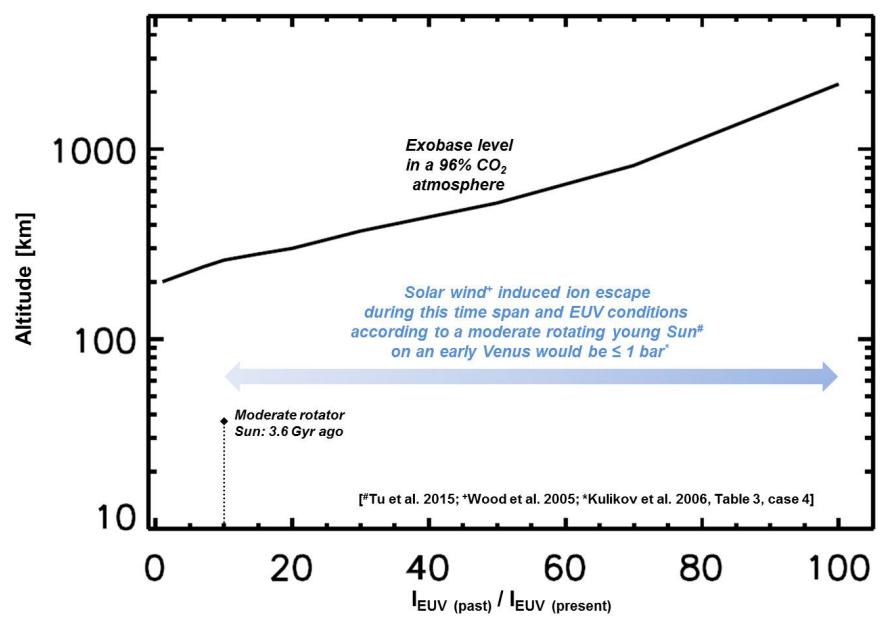

Fig. 12 Modeled Venus' upper atmosphere expansion and exobase level for a $96 \% \mathrm{CO}_{2}$ atmosphere as a function of solar EUV flux enhancement. The EUV flux enhancement corresponds to a moderately rotating young Sun. The vertical dotted line marks the 10 EUV flux enhancement factor that would have been reached about 3.6 Gyr ago. A $96 \% \mathrm{CO}_{2}$ atmosphere would lose a partial pressure of $\leq 1$ bar via solar wind induced ion pick up escape over about $4.5 \mathrm{Gyr}$ (after Kulikov et al. 2006).

surface conditions after this stage and the possible formation of a liquid water ocean depend on the initial volatile content and the orbit location. Depending on the formation time of the cloud cover, and its resulting albedo, Salvador et al. (2017) found that $\approx 0.3$ Earth ocean mass might have been enough that a water ocean formed at early Venus' surface.

Way et al. (2016) carried out 3D climate simulations by using solar spectral irradiance estimates for 2.9 and $0.715 \mathrm{Gyr}$ ago, an Earth ocean volume of liquid water, and various atmospheric compositions and cloud pattern. By using these parameters these authors found that under their assumptions Venus could have had moderate surface temperatures if the planet's rotation period was slower than $\approx 16$ Earth days, despite an incident solar flux 46-70\% higher than modern Earth receives.

However, in such scenarios, the hypothetical wet and "habitable" Venus, approaches at some time a moist stage and a runaway greenhouse effect finally transforms the planet to an extremely hot and dry body with growing solar luminosity (e.g., Rasool and de Bergh 1970; Kasting 1988; Salvador et al. 2017). One should also note that at ages as studied by Way et al. (2016) the EUV flux of the Sun would be too weak so that the water of an Earth-like ocean cannot escape easy to space when it is in the atmosphere. Although, $\mathrm{H}_{2} \mathrm{O}$ molecules will be dissociated in the upper atmosphere, the hydrogen escape rate would be too low $\approx 0.7$ - $3 \mathrm{Gyr}$ ago for an efficient escape of the left behind oxygen via hydrodynamic drag (Lichtenegger et al. 2016).

Moreover, according to Salvador et al. (2017) the surface temperatures of a steam atmosphere up to $\approx 1000$ bar partial pressure $\mathrm{CO}_{2}$ and water contents between $\approx 0.03-1.24$ equivalent amounts of an Earth ocean remains below $\approx 1800 \mathrm{~K}$, which is too cool for the formation of a magma ocean. Therefore, the surface oxidation of the large amount of left behind oxygen from an evaporating water ocean may be less efficient if early Venus formed a liquid ocean comparable with that of Earth. 
One may argue that the left behind oxygen can be lost from Venus since the past Gyr's by nonthermal ion escape. Kulikov et al. (2006) studied the nonthermal ion pick up escape rate of the Venusian thermosphere over the planet's history by applying a diffusivegravitational equilibrium and thermal balance model. These authors investigated the EUV heating by photodissociation and ionization processes, due to exothermic chemical reactions and cooling by $\mathrm{CO}_{2}$ IR emission in the $15 \mu \mathrm{m}$ band. As shown in Fig. 12, the simulation results of these authors yielded thermospheres with present-day exobase levels at $\approx 200 \mathrm{~km}$ and $\approx 2200 \mathrm{~km} 4.5 \mathrm{Gyr}$ ago, if one assumes that the young Sun was a moderate rotator. By using solar wind parameters (Kulikov et al. 2006: Case 4 in Table 3), in agreement with that of Johnstone et al. (2015a, 2015b), a $\mathrm{CO}_{2}$-dominated early Venus would have lost only about $\leq 1$ bar of its atmosphere during $4.5 \mathrm{Gyr}$ ago. From the low loss rates for escaping oxygen ions obtained by Kulikov et al. (2006) one can argue that there was no large amount of accumulated oxygen that was left behind from an evaporating surface ocean in Venus' atmosphere.

Therefore it is more likely, as suggest by Gillmann et al. (2009) and Lichtenegger et al. (2016), that hydrodynamic escape has dried up Venus atmosphere early in its history during $\leq 100 \mathrm{Myr}$, triggering the crystallization of the magma ocean (Marcq 2012; Elkins-Tanton 2012; Lebrun et al. 2013; Hamano et al. 2013; Massol et al. 2016; Salvador et al. 2017), and leaving no available water in the atmosphere to condense out and form an Earth-size water ocean. Earth, possibly obtained more water, and was most likely a subject to a weaker hydrodynamic escape, so that the planet remained wet after the Moon-forming impact and the crystallization of its final magma ocean. In such a scenario the oxygen left behind from the escaping hydrogen during the main hydrodynamic phase on Venus has been dissolved in the magma ocean, and lost through oxidation (Gillmann et al. 2009; Lebrun et at. 2013; Lichtenegger et al. 2016).

In such a case the dense Venus $\mathrm{CO}_{2}$ atmosphere doesn't result from an initial episode of runaway (or moist) greenhouse, but has been formed during the crystallization of the magma ocean, by progressive exsolution of carbon dioxide, at a time when the atmospheric partial pressure of water and hydrogen was of a few hundred bar (e.g., Ikoma and Genda 2006; Gillmann et al. 2009; Pierrehumbert and Gaidos 2011; Hamano et al. 2013; Massol et al. 2016; Stökl 2015 2016).

Because it is expected that early Earth had also a $\mathrm{CO}_{2}$-dominated atmosphere during the Hadean, the atmospheric pressure loss would have been even lower at $1 \mathrm{AU}$. In the case that the young Sun was a slow rotator, its EUV activity would have been lower than assumed in the study of Kulikov et al. (2006), which would have also resulted in lower atmospheric loss rates. This suggests that early Venus and Earth did not lose much of their initially outgassed atmospheric $\mathrm{CO}_{2}$ to space.

Fig. 13 shows an illustration of possible Venus' atmospheric evolution scenarios (see details in the figure caption). After the evaporation of a thin nebular-based $\mathrm{H}_{2}$ envelope that was necessary for the fractionation of the noble gases in the Venus atmosphere (Odert et al. 2018), an atmosphere related to magma ocean solidification was catastrophically outgassed. This atmosphere contained mainly $\mathrm{H}_{2} \mathrm{O}, \mathrm{CO}_{2}$ and in a lesser fraction, nitrogen. Weather early Venus had an ocean on its surface or not, the water evaporated due to a runaway greenhouse effect (e.g., Rasool and de Bergh 1970; Kasting 1988) and was lost from the atmosphere. However, during the escape process a huge amount of oxygen remains as a result of $\mathrm{H}_{2} \mathrm{O}$ photolysis and the hydrodynamically escape of the resulting hydrogen atoms in the atmosphere (Gillmann et al. 2009; Lichtenegger et al. 2016).

As discussed above the nonthermal loss to space was not efficient enough that the left behind oxygen could escape to space, only surface weathering and oxidation of the crust and 


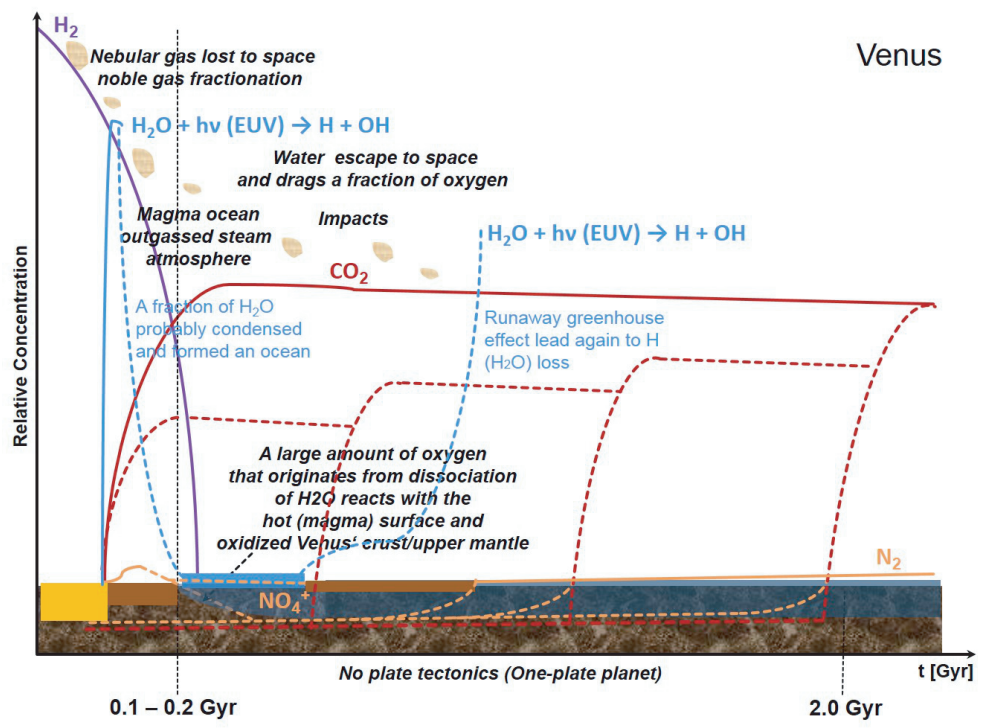

Fig. 13 Illustration of Venus's atmosphere evolution since its origin assuming that the planet never experienced plate tectonics or similar crustal recycling. After a thin nebular-based $\mathrm{H}_{2}$ envelope was lost (Odert et al. 2018), a global magma ocean solidified an outgassed volatiles (i.e. $\mathrm{H}_{2} \mathrm{O}, \mathrm{CO}_{2}$, etc.). A steam atmosphere originated. Depending on the blanketing effect, impact flux and possible cloud cover, and the outgassed $\mathrm{CO}_{2}$ and $\mathrm{H}_{2} \mathrm{O}$ content (Salvador et al. 2017), water could condense and formed a surface ocean (blue dashed line), or remained for ever in vapor form. If an early ocean originated the water evaporated later by a runaway greenhouse effect (Rasool and de Bergh1970; Kasting and Pollack 1983; Kasting et al. 1984a; Kasting 1988; Way et al. 2016; Salvador et al. 2017). Hydrogen of dissociated $\mathrm{H}_{2} \mathrm{O}$ molecules escaped thermally to space but the left behind oxygen should have been weathered into the surface, or also lost to space, via nonthermal escape processes. Discussed in the main text the later process was most likely not efficient enough. Atmospheric nitrogen was most likely weathered into the surface via abiotic reduction processes during the hot and magmatic surface conditions (Wordsworth 2016a). On the other hand, if no liquid surface ocean formed (Lebrun et al. 2013; Hamano et al. 2013; Massol et al. 2016), the hydrogen of dissociated $\mathrm{H}_{2} \mathrm{O}$ molecules escaped efficiently via hydrodynamic escape to space and the left behind oxygen oxidized the magmatic crust and upper mantle (Gillmann et al. 2009; Lebrun et at. 2013; Lichtenegger et al. 2016). Because of the lack of liquid water atmospheric $\mathrm{CO}_{2}$ could not be weathered into carbonates, remained in the atmosphere and/or was also degassed with a minor fraction of $\mathrm{N}_{2}$ episodically by super volcanos or resurfacing events since 4.5 Gyr ago.

upper mantle could have been an efficient surface sink. As long as a magma ocean formed the remaining oxygen and atmospheric nitrogen could have been incorporated into Venus' hot magmatic crust, where the oxygen oxidized the upper mantle material (e.g., Gillmann et al. 2009; Lichtenegger et al. 2016; Wordsworth 2016a). Due to that, the above described scenarios would work better if Venus' never originated an "Earth-like" global liquid water ocean on its surface, or if such an ocean evaporated within the first $100 \mathrm{Myr}$ after the planets origin, since later the EUV flux of the early Sun would be too weak for driving an efficient atmospheric escape.

The highly oxidized surface material could then be mixed with reduced, nitrogen-rich material in the deep interior. This could also have caused increased outgassing of $\mathrm{N}_{2}$ over time even if the oxidation state of the upper mantle remained constant (Wordsworth 2016a) and can easily explain the 3.4 times greater atmospheric $\mathrm{N}_{2}$ of Venus inventory compared to the Earth. Due to upper atmosphere cooling from the $\mathrm{CO}_{2}$-dominated atmosphere, the 


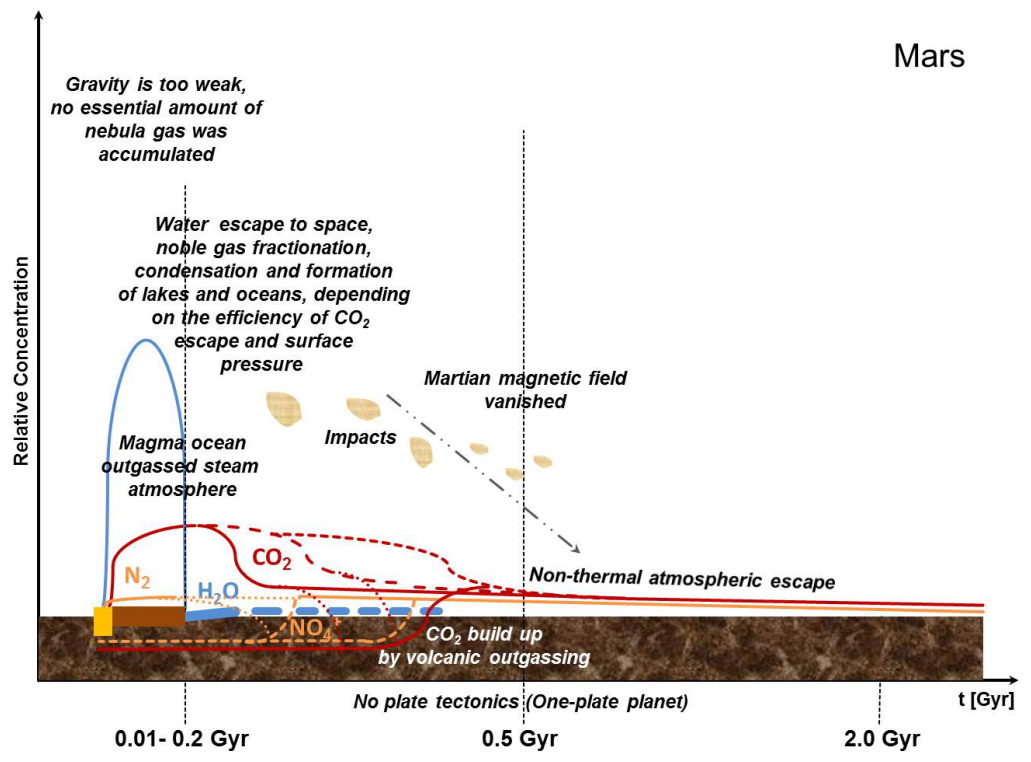

Fig. 14 Illustration of Mars' atmosphere evolution since its origin. Depending on the amount of outgassed $\mathrm{CO}_{2}$ and the early Sun's EUV flux history, Mars could have had permanently or episodically large lakes or even an ocean on its surface during the first 100-300 Myr. Depending on the degree of oxidation of Mars' crust and upper mantle nitrogen could have been outgassed during the first Gyr via volcanos but experienced efficient atmospheric escape to space. Subsequent to $4.0 \mathrm{Gyr}$ ago the $\mathrm{CO}_{2}$ partial surface pressure decreased by thermal escape and since about 3.5 - 4.0 Gyr ago by nonthermal escape and surface weathering.

outgassed nitrogen was not lost to space during the active young Sun period, and remained in the atmosphere until today.

As pointed out before, the growth time of the smaller planet Mars is estimated to be of the order of a few Myr (e.g., Hansen 2009; Dauphas and Pourmand 2011; Walsh et al. 2011; Brasser 2013), indicating that Mars is a large planetary embryo that never grew to a "real" larger Venus- or Earth-like planet (Brasser 2013). Related to its formation (e.g.,Walsh et al. 2011; Brasser et al. 2017) and geological surface features (i.e. wrinkle ridges, etc.), Mars' mantle, appears to be more reduced than that of Earth (Montesi and Zuber 2003; Stanley et al. 2011). Therefore, hydrogen and water could have been outgassed efficiently after magma ocean solidification and impacts (e.g., Ramirez et al. 2014). For early Mars, even if the planet was water-rich in its past, such a small body would have lost its ability to activate plate tectonics very quickly. Maybe Mars had once a short period of proto-plate tectonics (Frey 2006; Yin 2012), but this most likely stopped during the pre-Noachian period together with the Martian magnetic dynamo (Dehant et al. 2007, and references therein; Lillis et al. 2008, 2013; Robbins et al. 2013). However, even if early Mars had experienced this primitive stage of plate tectonics this process would have stopped $>4.0$ Gyr ago.

Mars' atmosphere evolution is illustrated in Fig. 14. Due to the planet's low gravity, no essential nebular-based $\mathrm{H}_{2}$ envelope could have accumulated (Erkaev et al. 2014; Stökl 2016; Odert et al. 2018). After Mars' final magma ocean solidified, the outgassed steam atmosphere reached its condensation phase at about $\geq 0.1$ Myr (Lebrun et al. 2013; Massol et al. 2016). Depending on the absolute amounts of the catastrophically outgassed volatiles (Elkins-Tanton 2008; Erkaev et al. 2014) and impacts (e.g., Segura 2002; 2008; 2012; Rodriguez et al. 2015; 
Iijima et al. 2017; Palumbo and Head III 2018), available greenhouse gases (i.e., $\mathrm{CO}_{2}, \mathrm{CH}_{4}$, $\mathrm{H}_{2}$, etc.) (Kasting 1991; Forget et al. 2013; Ramirez et al. 2014; Wordsworth et al. 2013; Kerber et al. 2015; Wordsworth 2016b; Fairén 2017; Iijima et al. 2017) and the Sun's EUV flux evolution, the surface partial pressures of $\mathrm{H}_{2} \mathrm{O}, \mathrm{CO}_{2}, \mathrm{CO}, \mathrm{N}_{2}$, and $\mathrm{O}$, large lakes, or an ocean could have been episodically present on Mars' surface during the first $\approx 100-300$ Myr in the early Noachian (e.g., Dohm et al. 2008; Buhler et al. 2014; Palumbo et al. 2014; Rodriguez et al. 2015; Rosenberg and Head III 2015; Amerstorfer et al. 2017; Fairén 2017; Luo et al. 2017; Palumbo and Head III 2018).

The initial $\mathrm{CO}_{2}$ partial surface pressure decreased via EUV-driven thermal escape to low values until about 4.0 - 4.2 Gyr ago (Tian et al. 2009; Amerstorfer et al. 2017). Moreover, Zahnle et al. (2008) studied the photochemical stability of the Martian $\mathrm{CO}_{2}$ atmosphere during the planet's history. By applying a 1D photochemical model these authors found that a thick cold, dry $\mathrm{CO}_{2}$ atmosphere is photochemically unstable so that $\mathrm{CO}_{2}$ would convert into $\mathrm{CO}$. This possible photochemical instability of a cold dense $\mathrm{CO}_{2}$ atmosphere on early Mars, and the likelihood that $\mathrm{CO}$ was more abundant compared to $\mathrm{CO}_{2}$, would have important implications for early Mars, such as higher atmospheric escape rates (Tian et al. 2009) caused by reduced $\mathrm{CO}_{2}$-related IR-cooling of the upper atmosphere.

Volcanos and impacts during the late heavy bombardment (e.g., Bottke et al. 2012) period resulted in the build-up of a secondary $\mathrm{CO}_{2}$ atmosphere with a surface partial pressure that reached several hundred mbar (Grott et al. 2011; Lammer et al. 2013). Recent paleopressure studies of Kite et al. (2014) estimated upper values of the atmospheric surface pressure constraints of about $0.9-1.9$ bar, from the size-frequency distribution of small ancient craters with ages $>3.6 \mathrm{Gyr}$ within river deposits in the Aeolis region. Lower atmospheric surface pressure values of $\geq 0.5$ bar for ages of $\approx 4$ Gyr ago were estimated recently by Kurokawa et al. (2017) from $\mathrm{N}_{2}$ and Ar isotopic composition measurements.

According to the study of Amerstorfer et al. (2017) one can expect that nonthermal atmospheric escape processes prevented the build-up of an atmosphere with the upper surface pressure values inferred from the paleopressure study. These results are in agreement with earlier studies such as those of Fox (1993), Jakosky et al. (1994), and Hutchins and Jakosky (1996).

Depending on the radiation evolution of the young Sun, atmospheric $\mathrm{CO}_{2}$, or carbon related atmospheric compounds with surface partial pressures ranging from $\approx 200-400$ mbar were able to escape to space during the last $4 \mathrm{Gyr}$ by suprathermal atom escape (Amerstorfer et al. 2017). One can also expect that solar wind induced ion escape contributed to the atmosphere loss (Bösswetter et al. 2010) so that in agreement with Kurokawa et al. (2018), Mars may have lost $\geq 0.5$ bar of its atmosphere by nonthermal atmospheric loss processes (i.e., ion pick up, sputtering, escape of suprathermal atoms, impacts) and surface weathering processes of $\mathrm{CO}_{2}$, such as serpentinization (e.g., Chassefiére and Leblanc 2011) since $\approx 4$ Gyr ago.

\section{Origin and evolution of atmospheric $\mathrm{N}_{2}$}

The earliest nitrogen inventory was delivered to Earth, Mars, and Venus during the planetary accretion phase, by planetesimals that originated behind the ice-line. These bodies contained ices with trapped $\mathrm{NH}_{3}$ and $\mathrm{CH}_{4}$ in the form of clathrate, amino acids, and other organic compounds (e.g., Canfield et al. 2010, Cloud 1968, Holland 1984). Impact shock experiments on carbonaceous chondrites indicate once the proto-Earth reached $\approx 30 \%$ of its final radius, 
the devolatilisation of minerals was very effective (Tyburczy et al. 1986), such that impactdelivered nitrogen would have been thermalized and degassed directly into the atmosphere. Moreover, the impacting bodies could also have devolatilised any crustal nitrogen that was present in the impact area (Wordsworth 2016a).

\subsection{Prebiotic nitrogen delivery}

Until recently, based on the abundances of Vanadium (V) and Chrome (Cr) in ancient basalts and komatiites most researchers expected that volcanic gases on the Archean Earth would not have been more reduced than today (e.g., Delano 2001; Canil 2002; Li and Lee 2004). However, Aulbach and Stagno (2016) and Nicklas et al. (2016) suggest that mantle oxidation could have been slightly lower than today, but probably not low enough to support efficient outgassing of $\mathrm{NH}_{3}$. The early Hadean crustal and atmospheric conditions would have been more reducing compared to conditions during the Archean and later in Earth's history, due to captured nebular gas, serpentinization and chemical equilibrium of the accreting chondritic material (Schaefer and Fegley 2010). Under such conditions, nitrogen presumably would have been thermodynamically favored in the form of $\mathrm{NH}_{3}$ near the surface (Wordsworth 2016a), which could have built up to a few tenths of a percent in Earth's atmosphere if the surface is shielded from the solar UV flux (Holland 1984; Stevenson 1983a; Stevenson 1983b; Stevenson 1990, Turner et al 1990). If this estimate is applied to the total partial surface pressure expected from the outgassing of the final magma ocean of $\approx 400-650$ bar $\left(\mathrm{H}_{2} \mathrm{O}, \mathrm{CO}_{2}\right)$ (Elkins-Tanton 2008; Elkins-Tanton 2012), then early Earth could already have had a few 100's of mbars of $\mathrm{N}_{2}$ in its steam atmosphere as early as 4.5 Gyr ago.

Nitrogen isotope data $\delta^{15} \mathrm{~N}=\left(\left(\left({ }^{15} \mathrm{~N} /{ }^{14} \mathrm{~N}\right) /\left({ }^{15} \mathrm{~N} /{ }^{14} \mathrm{~N}\right)\right)_{\text {atm }}-1\right) \times 1000$ measured in \%o support a carbonaceous chondrite origin for the majority of nitrogen on the terrestrial planets (Owen et al. 2001; Marty 2012) (Fig. 15). However, atmospheric nitrogen on Earth and Venus do not exhibit the same "strong" ${ }^{15} \mathrm{~N}$-enrichment found in the atmosphere on Mars (Füri and Marty 2015) (Fig. 15). Higher ${ }^{15} \mathrm{~N}$ in atmospheric $\mathrm{N}_{2}$ on Mars is generally thought to indicate that Mars lost a larger amount of its nitrogen inventory to space, which resulted in a strong atmospheric enrichment of the heavier ${ }^{15} \mathrm{~N}$ isotope (Füri and Marty 2015). Since Mars has a $\mathrm{CO}_{2}$-dominated atmosphere that protects the bulk atmosphere against upper atmospheric expansion and strong thermal escape during the Hesperian up to present, enrichment of ${ }^{15} \mathrm{~N}$ isotopes most likely occurred earlier i.e. when the young Sun's EUV flux was higher and because of Mars' low gravity.

Venus is thought to have been similarly protected by its $\mathrm{CO}_{2}$-enriched atmosphere. In the case of such a massive planet, its $\mathrm{CO}_{2}$ atmosphere would have acted as an efficient IRcoolant in the upper atmosphere which would have protected the atmosphere against EUV heating, expansion and strong thermal atmospheric escape (Kulikov et al. 2007; Kulikov et al. 2006; see also sub Sect. 4.2). $\mathrm{A} \mathrm{CO}_{2}$-dominated atmosphere would also have shielded $\mathrm{NH}_{3}$ molecules near the surface from photolysis and would have caused enhanced ammonia rainout rates. This process could have additionally decreased raindrop $\mathrm{pH}$ and consequently the oceanic $\mathrm{pH}$, which would have eventually favored the conversion of nitrogen in an atmosphere-ocean system from gaseous $\mathrm{NH}_{3}$ to aqueous $\mathrm{NH}_{4}^{+}$(Wordsworth 2016a).

In addition, abiotic reduction of atmospheric $\mathrm{N}_{2}$ could have been very effective up until the later stages of accretion on early Earth, Mars, and Venus. These young planets likely repeatedly experienced magma oceans, and thus hot and extreme surface conditions, during the first hundred Myr of their history (Fig. 9). Reduced atmospheric nitrogen could have been transported to the mantle very quickly under the high surface temperatures $(>800 \mathrm{~K})$ 


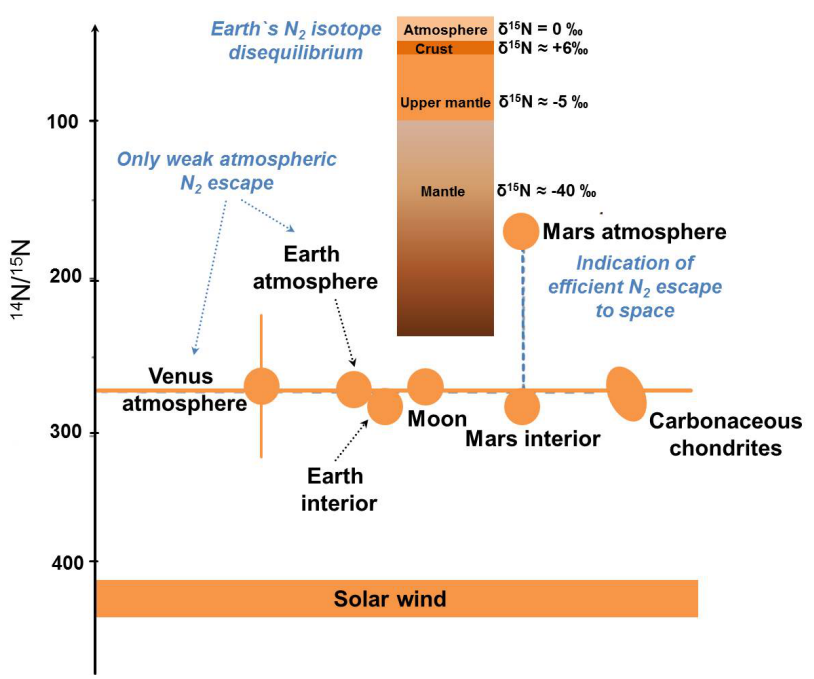

Fig. $15{ }^{14} \mathrm{~N} /{ }^{15} \mathrm{~N}$ isotope variations in the atmospheres and interior (mantle) of Venus, Earth-Moon, and Mars are comparable with that of carbonaceous chondrites and different than that observed in the solar wind or in the atmospheres of the gas giants. Venus' atmospheric ratio has large error bar (vertical line) but is most likely not much different compared to Earth's atmosphere/interior, the Moon, Mars' interior and chondrites. Mars' atmospheric nitrogen is strongly enriched in ${ }^{15} \mathrm{~N}$ while Earth's atmospheric nitrogen shows only a small enrichment (after Füri and Marty 2015). The insert illustrates the so-called nitrogen isotope disequilibrium between the mantle depleted in ${ }^{15} \mathrm{~N}$ and atmosphere/crust/upper mantle (enriched in ${ }^{15} \mathrm{~N}$ ) reservoirs of present Earth (after Cartigny and Marty 2013).

that could have developed (Wordsworth 2016a). Therefore, the dissolution of nitrogen in early magma oceans or hot magmatic surface environments could have led to a significant incorporation of nitrogen into the mantle from very early in Earth's history (Marty 2012).

Present Earth's nitrogen reservoirs are currently in isotopic disequilibrium. With Earth's atmospheric $\delta^{15} \mathrm{~N}$ defined as $0 \%$, the crust is generally more ${ }^{15} \mathrm{~N}$-enriched (with $\delta^{15} \mathrm{~N}+6 \%$ ), the upper mantle is slightly ${ }^{15} \mathrm{~N}$-depleted (with $\delta^{15} \mathrm{~N}-5 \%$ ), and the deeper mantle is strongly ${ }^{15} \mathrm{~N}$-depleted (with $\delta^{15} \mathrm{~N}-40 \%$ ) (Fig. 15). This nitrogen isotope disequilibrium could have been caused by $\delta^{15} \mathrm{~N}$ fractionation via atmospheric escape, mantle-core segregation, late accretion of ${ }^{15} \mathrm{~N}$-rich volatiles to form the atmosphere and oceans, and/or the recycling of ${ }^{15} \mathrm{~N}$-depleted sedimentary nitrogen deposited during Earth's past (Cartigny and Marty 2013). Possible causes of this disequilibrium are further addressed in Sect. 6.2 below.

\subsection{Earth's surficial nitrogen cycles}

Fig. 16 illustrates the major processes which cycle nitrogen between the atmosphere and Earth's interior, as modulated by the biosphere (e.g., Jacob 1999; Galloway, 2003; Fowler et al. 2013; Stüeken et al. 2016a; Stüeken et al. 2016b; Zerkle and Mikhail 2017). Table 3 summarizes the main sources and sinks of nitrogen mass in the present Earth atmosphere, soil, land biota, ocean and ocean biota, along with mass rates per year from the atmosphere into the surface/interior and from the surface into the atmosphere by neglecting industrial fertilizers (Galloway 2003, and fluxes after Fowler et al. 2013). At present, $\mathrm{N}_{2}$ is the main nitrogen species in Earth's atmosphere, and at 0.79 bar accounts for around $78 \%$ of the total atmosphere. The transformation of inert atmospheric $\mathrm{N}_{2}$ to biologically available nitrogen 
Table 3 Main sources and sinks of nitrogen mass (in $\mathrm{Tg}=10^{12}$ ) and mass rates per year (in $\mathrm{Tg} / \mathrm{yr}$ ) from the atmosphere into the surface/interior and from the surface into the atmosphere in the present Earth atmosphere, soil, land biota, ocean and ocean biota. Sinks caused by industrial fertilizers are not given (Galloway 2003, and fluxes after Fowler et al. 2013).

\begin{tabular}{l|l|l|l|l}
\hline Atmosphere & Soil & Land biota & Ocean & Ocean biota \\
\hline $3.95 \times 10^{9} \mathrm{Tg}$ & $1 \times 10^{9} \mathrm{Tg}$ & $1 \times 10^{4} \mathrm{Tg}$ & $2.06 \times 10^{7} \mathrm{Tg}$ & $5 \times 10^{2} \mathrm{Tg}$ \\
\hline Biofixation & - & $-120 \mathrm{Tg} / \mathrm{yr}$ & - & $-140 \mathrm{Tg} / \mathrm{yr}$ \\
Rain/Lightning & $-70 \mathrm{Tg} / \mathrm{yr}$ & - & $-30 \mathrm{Tg} / \mathrm{yr}$ & - \\
Denitrification & - & $110 \mathrm{Tg} / \mathrm{yr}$ & - & $190 \mathrm{Tg} / \mathrm{yr}$ \\
Biomass burning & - & $5 \mathrm{Tg} / \mathrm{yr}$ & - & - \\
Surface release & $78 \mathrm{Tg} / \mathrm{yr}$ & - & $15 \mathrm{Tg} / \mathrm{yr}$ & \\
\hline
\end{tabular}

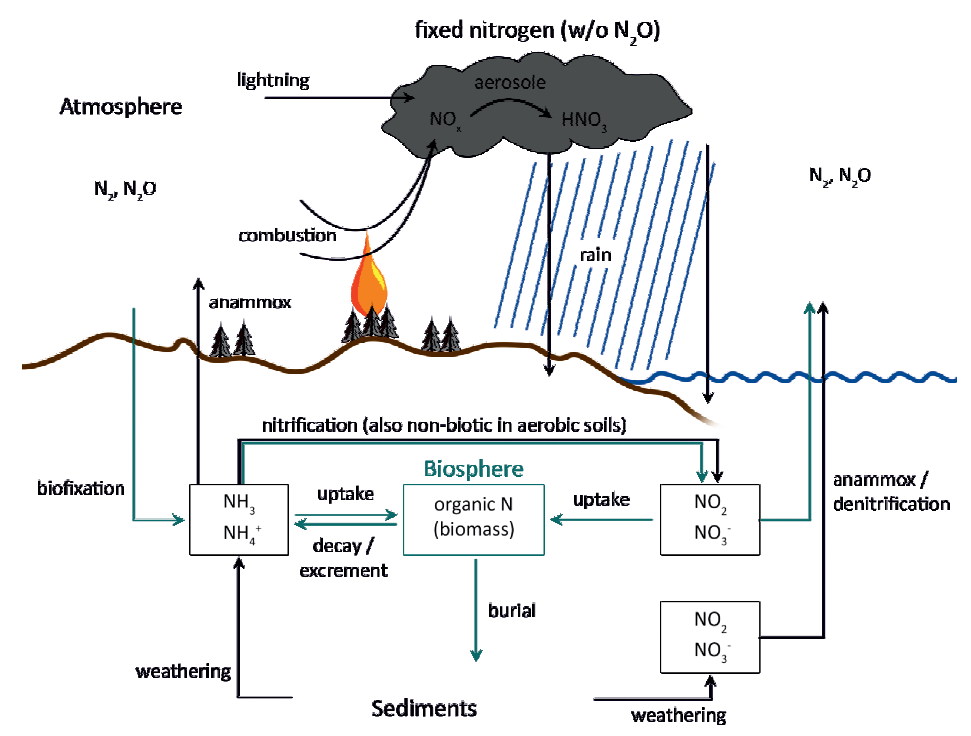

Fig. 16 Illustration of the main processes that are related to present Earth's $\mathrm{N}_{2}$ budget and cycle, including estimates of nitrogen inventories in the atmosphere, deep ocean and the Lithosphere and the mantle (e.g., Schlesinger 1997; Jacob 1999; Galloway 2003; Goldblatt et al. 2009, and references therein; Fowler et al. 2013; Stüeken et al. 2016a; Stüeken et al. 2016b; Zerkle and Mikhail 2017) (courtesy of L. Strauß).

is called $\mathrm{N}_{2}$ fixation, or diazotrophy. In Earth's biosphere, prokaryotes (both Bacteria and Archaea) can reduce atmospheric $\mathrm{N}_{2}$ to organic nitrogen in a reduced form. On land, soil diazotrophs also supply fixed nitrogen to the roots (rhizosphere) of plants, which form the base of the terrestrial trophic structure. These life forms excrete the nitrogen or die, leading to the buildup of organic nitrogen in soils and sediments. Organic nitrogen is rapidly assimilated and recycled in the surface ocean, soils, and sediments, or remineralized as $\mathrm{NH}_{4}^{+}$. A number of prokaryotes can also use dissolved inorganic nitrogen compounds in energy-yielding reactions.

In the presence of oxygen, microorganisms can oxidize ammonium to nitrite $\mathrm{NO}_{2}^{-}$and to nitrate $\mathrm{NO}_{3}^{-}$, during the step-wise process of nitrification (Carlucci and McNally 1969). Nitrate is mobile in soils and aqueous environments, and can also be assimilated into organic nitrogen by organisms. Under conditions where $\mathrm{O}_{2}$ is depleted, including modern oxygen minimum zones, anaerobic prokaryotes can also use nitrate as an electron acceptor for the 
oxidation of organic carbon to $\mathrm{CO}_{2}$. Anaerobic respiration of nitrate (termed denitrification) returns fixed nitrogen back to the atmosphere as $\mathrm{N}_{2}$. Fixed nitrogen can also be returned to the atmosphere via the anaerobic oxidation of ammonium with nitrite (termed anammox; Dalsgaard et al 2005). Some fixed nitrogen remains buried in the sediments, to be released during metamorphism (e.g., Haendel et al. 1986) or subducted. Subduction of N-bearing sediments and altered oceanic lithosphere constitutes the primary flux of surficial nitrogen into the deep Earth (Busigny et al. 2011, Halama et al. 2014).

The above discussion highlights the importance of biological reactions in the cycling of nitrogen through Earth's oceans, atmosphere, and sediments (e.g., Cartigny and Marty 2013, Stüeken et al. 2016a, Zerkle and Mikhail 2017). As life and its environments have evolved over $<3$ Gyr of Earth history, this dynamic interplay between the geosphere, biosphere, and atmosphere has dramatically altered the cycling of nitrogen over geologic time. Direct data for the partial pressure of atmospheric $\mathrm{N}_{2}$ over time are limited, but available data support large swings in atmospheric $\mathrm{pN}_{2}$ since the Archean (Marty et al. 2013, Som et al. 2016), suggesting systematic (or punctuated) changes in nitrogen cycling over Earth's history.

Biological $\mathrm{N}_{2}$ fixation currently provides the primary flux of $\mathrm{N}_{2}$ from the atmosphere to the mantle, via organic matter burial and subduction. Photochemical fixation of atmospheric nitrogen to $\mathrm{HCN}$ has been proposed as an important source for prebiotic chemistry in Earth's early atmosphere (Zahnle 1986), but would only have been substantial under conditions of high atmospheric methane fuelled by a globally efficient biosphere (Tian et al 2011, Wordsworth and Pierrhumbert 2013). An additional abiotic pathway for fixing atmospheric $\mathrm{N}_{2}$ is by high-temperature oxidation of $\mathrm{N}_{2}$ to NO (Kasting and Siefert 2001; NavarroGonzalez et al. 2001). This reaction occurs during combustion or lightning, followed by oxidation of $\mathrm{NO}$ to $\mathrm{HNO}_{3}$ in the atmosphere, which is $\mathrm{H}_{2} \mathrm{O}$-soluble and scavenged by rain. However, on a prebiotic Earth with modern-style tectonics, circulation of seawater through mid ocean ridges could have reduced $\mathrm{NO}_{x}$ to $\mathrm{N}_{2}$ or $\mathrm{NH}_{3}$, which could then be photochemically converted back to $\mathrm{N}_{2}$, for a possible zero-sum gain of fixed nitrogen (Kasting et al. 1993a).

The timing of the evolution of $\mathrm{N}_{2}$ fixation is unknown, but the sedimentary $\delta^{15} \mathrm{~N}$ record provides evidence of active diazotrophy as far back as $\approx 3.2$ Gyr ago (Stüeken et al. 2015), and phylogenetic reconstructions of the requisite genes support an early emergence (Boyd and Peters 2013) possibly even in the last common ancestor (Weiss et al. 2016). Assuming no other nutrients were limiting, the evolution of nitrogen fixation would have allowed for significant biospheric expansion (Haqq-Misra et al. 2008; Kharecha et al. 2005) and progressive drawdown of atmospheric $\mathrm{N}_{2}$ from the atmosphere into the biosphere-ocean-sediment system. Microbially facilitated fluxes of $\mathrm{N}_{2}$ from the biosphere back to the atmosphere in modern oxygen minimum zones counteract the drawdown of $\mathrm{N}_{2}$ via fixation and burial, such that Earth's atmosphere is not depleted in $\mathrm{N}_{2}$ (e.g., Cartigny and Marty 2013). However, these nitrogen loss processes (denitrification and anammox) both require nitrogen in an oxidized form (nitrate and nitrite, respectively), which would have been limited in the low oxygen environments of the early- to mid-Archean.

Small increases in sedimentary $\delta^{15} \mathrm{~N}$ values alongside additional geochemical indicators of local oxygenation have been interpreted as evidence for the evolution of coupled nitrification-denitrification by $\approx 2.7$ Gyr ago (Busigny et al. 2013; Garvin et al. 2009; Godfrey and Falkowski 2009). These aerobic N cycling processes appear to have been spatially and temporally restricted before the Paleoproterozoic (Stüeken et al. 2016a). However, the widespread oxygenation of Earth's surface environments during the Great Oxidation Event $(\mathrm{GOE}) \approx 2.3$ Gyr ago (Luo et al. 2016) dramatically increased the availability of oxidized nitrogen compounds in surface waters (Zerkle et al. 2017). These oxidized nitrogen compounds could have facilitated widespread $\mathrm{N}_{2}$ loss from the biosphere back to the atmosphere 
(e.g., Fennel et al. 2005) in the ferruginous anoxic deepwaters that prevailed throughout the Proterozoic (Poulton and Canfield 2011). This change in the biogeochemical nitrogen cycle could have caused an inflection point in the overall $\mathrm{N}$ balance of Earth's surface, if the flux of $\mathrm{N}_{2}$ from the oceans to the atmosphere was greater than or equal to the flux of $\mathrm{N}_{2}$ from the atmosphere to the sediments for the first time in Earth history. This scenario would have halted progressive drawdown of atmospheric $\mathrm{N}_{2}$, a process which could have been either dampened or exacerbated by changes in $\mathrm{N}_{2}$ outgassing from the mantle (e.g., Zerkle and Mikhail 2017).

5.3 Nitrogen speciation in upper mantle fluids and consequences for the evolution of $\mathrm{N}_{2}$ atmospheres

As discussed above, on a prebiotic Earth the most effective drawdown of atmospheric nitrogen occurred via direct dissolution of reduced nitrogen into magma oceans during the early Hadean, when conditions were extremely hot and reducing. Accordingly, the Hadean Earth could have had most nitrogen in its mantle, with negligible or only low amounts of $\mathrm{N}_{2}$ in the atmosphere (Wordsworth 2016a). Once life (and $\mathrm{N}_{2}$ fixation) arose, the biosphere would have regulated the amount of nitrogen buried in sediments hence the amount subducted, via the processes described above. Changes in volcanic outgassing of nitrogen from the upper mantle during the Precambrian could have further modulated atmospheric $\mathrm{N}_{2}$.

The current amount of nitrogen in Earth's crust and mantle is highly uncertain and estimated between surface partial pressures of 0.32 - 5.6 bar (e.g., Goldblatt et al. 2009, Johnson and Goldblatt 2015; Marty 1995; Marty and Dauphas 2003). The primary control on whether elements are degassed versus stored in the mantle is their compatibility with mantle minerals relative to melts and fluids. The speciation of nitrogen in mantle fluids is not well-constrained. Recent experimentally- and theoretically-determined equilibrium constants have shown that temperature, pressure, oxygen fugacity, nitrogen concentration, and $\mathrm{pH}$ all exert an important control on nitrogen speciation under mantle conditions (Mikhail et al. 2017). The overall effect of these variables on the distribution of nitrogen in the mantle are difficult to decipher. However, if we consider mantle redox as an example, models varying $\mathrm{f}_{\mathrm{O}_{2}}$ have shown that ammonium should be the dominant nitrogen species in aqueous fluids in the uppermost mantle, while molecular nitrogen $\left(\mathrm{N}_{2}\right)$ should dominate aqueous fluids in the more oxidized mantle wedge (Li and Keppler 2014; Mikhail and Sverjensky 2014). This distribution has important implications for the flux of $\mathrm{N}_{2}$ from the mantle, since $\mathrm{N}_{2}$ is highly conducive to outgassing, while $\mathrm{NH}_{4}^{+}$is moderately compatible in $\mathrm{K}, \mathrm{Ca}$, and $\mathrm{Na}$ bearing silicates like phlogopite and clinopyroxene and can be stored in the deep Earth.

Accordingly, multiple lines of evidence now point to the existence of a large deep mantlebased nitrogen reservoir (Li and Keppler 2014; Li et al. 2013; Mikhail and Sverjensky 2014; Watenphul and Wunder 2010). This deep Earth nitrogen reservoir can help to resolve the missing nitrogen conundrum, whereby the abundance of nitrogen in the bulk silicate Earth appears to be significantly lower than that of other volatile elements, but only if this calculation uses $\mathrm{N}_{2}$ /Ar ratios and assumes nitrogen behaves like a noble gas (Halliday 2013; Marty 2012). In addition, Earth's present atmosphere shows a visible enrichment of $\mathrm{N}_{2}$ relative to the nonradiogenic primordial noble gases compared to Venus and Mars (Fig. 17) (Hoffman et al. 1980; Mahaffy et al. 2013; Porcelli and Pepin 2003). This $\mathrm{N}_{2}$ enrichment in Earth's atmosphere requires a mechanism that can fractionate the nitrogen from the noble gases during volcanic outgassing by a process that is not active on the two other planets. 


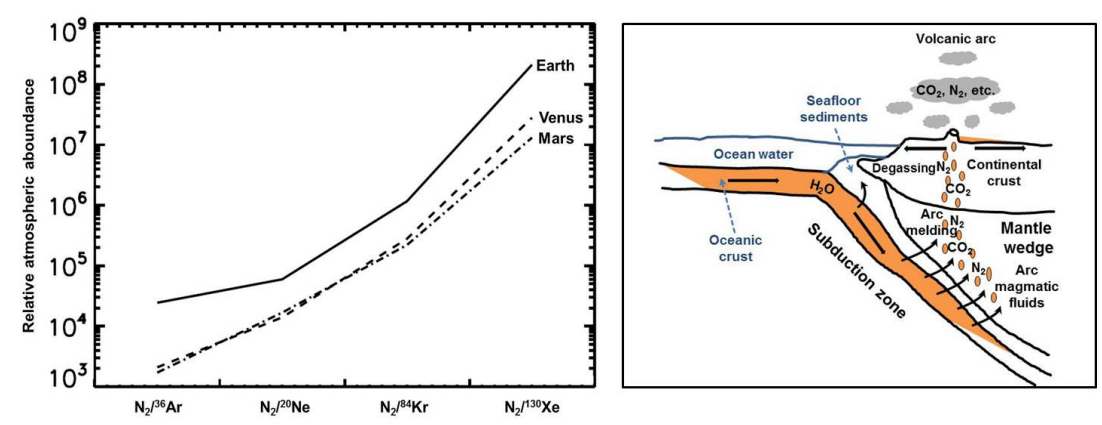

Fig. 17 Left: The atmospheric abundance of atmospheric $\mathrm{N}_{2}$ (Mikhail and Sverjensky 2014) relative to the primordial nonradiogenic noble gases of Venus (Hoffmann et al. 1980; Porcelli and Pepin 2003), Earth (Porcelli and Pepin 2003) and Mars (Mahaffy et al. 2013). Right: Illustration of a schematic vertical crosssection through a subduction zone, displaying the geochemical cycle of fluids and outgassing related to mantle wedge areas.

Fig. 17 shows an illustration of the tectonic processes that could favour the formation of an $\mathrm{N}_{2}$-rich atmosphere relative to the primordial noble gases on Earth (e.g., Mikhail and Sverjensky 2014). The mantle wedge area is shown as the triangular shaped piece of mantle that lies above a subducting tectonic plate and below the overriding plate. Subduction of oceanic lithosphere carries oxidized surface rocks and large amounts of water into Earth's upper mantle (Hirschmann 2010). This water lowers the melting temperature of the mantle wedge above (Kelley et al. 2010). The rocks, including sediments and hydrothermally altered basalts, are rich in water, which is also released into the overlying mantle wedge. The melting of the mantle wedge then contributes to depressurization due to the flow in the wedge. Moreover, the melt gives rise to associated volcanism and preferred outgassing of nitrogen in the form of $\mathrm{N}_{2}$ on the Earth's surface (Hirschmann 2010). Under this scenario, $\mathrm{NH}_{4}^{+}$is moderately compatible in the upper mantle minerals and unconducive to nitrogen degassing. The mantle was likely more reduced at 4.5 Gyr ago (e.g., Frost et al 2008, Kasting 1992) and evolved towards a more oxidized state due to the release of reduced volcanic gases and the escape of hydrogen that left behind oxygen from dissociation of $\mathrm{H}_{2} \mathrm{O}$ molecules. The timing of the onset of subduction-zone plate tectonics on Earth is controversial (e.g., Turner et al. 2014), but most estimates agree that at least some form of plate tectonics was in place by $\approx 3$ Gyr ago (Fig. 10). $\mathrm{N}_{2}$ degassing would have followed once subduction zones began injecting oxidants, like $\mathrm{Fe}_{3}^{+}$or $\mathrm{S}_{6}^{+}$into the mantle (Kelley and Cottrell 2009). However, the overall oxidizing potential of the subducted sediments and altered oceanic crust must also have changed over geological time due to the progressive oxidation of Earth's surface (e.g., see review in Lyons et al. 2014). In particular, widespread oxygenation of Earth's atmosphere and surface oceans occurred $\approx 2.3 \mathrm{Gyr}$ ago during the GOE (e.g., Luo et al. 2016), although deep oceans remained largely anoxic and Fe-rich until the late Neoproterozoic (e.g., Poulton and Canfield 2011). Concomitant oxygenation of subducted sediments could have generally increased the flux of $\mathrm{N}_{2}$ from the mantle over time, resulting in Earth's currently $\mathrm{N}_{2}$-rich atmosphere (Mikhail and Sverjensky 2014). 
6 Response of Earth's $\mathrm{N}_{2}$ atmosphere against the Sun's EUV flux during the Archean

The expected solar EUV flux for a slow, moderate, and fast rotating young solar like Gstar during the late Hadean, Archean, and early Proterozoic is shown in Fig. 18. Over this period the atmospheric $\mathrm{CO}_{2}$ was slowly weathered into the planet's interior as e.g. studies by Kanzaki and Murakami (2015) are indicating. However, the partial surface pressure of $\mathrm{CO}_{2}$ was still as high as $85-510$ PAL for $\approx 2.77 \mathrm{Gyr}$ and 20-620 PAL for $\approx 2.08 \mathrm{Gyr}$ (Kanzaki and Murakami, 2015). Nevertheless $\mathrm{N}_{2}$ could have become the dominant atmospheric species during the early to mid-Archean, depending on whether a nitrogen fraction remained in the atmosphere from volatile-rich impactors during the Hadean, and/or on when nitrogen started to be outgassed efficiently because of oxidized mantle wedge conditions and related volcanic activity.

\subsection{Thermal and nonthermal nitrogen escape}

According to studies by Tian et al. (2008a, 2008b) related to the EUV exposure of Earth's upper atmosphere when the solar EUV flux exceeds a critical value $(\approx 5 \mathrm{EUV})$ the upper atmosphere (present-day Earth composition) switches from the hydrostatic to the hydrodynamic regime. This process is accompanied by faster atmospheric bulk flow velocities, which approach the escape velocity for EUV flux values $\approx 10$ times higher than today. Fig. 19 suggests that, for an EUV flux enhancement of $\approx 9$ times higher modern day, the exobase of Earth's atmosphere assuming a $\mathrm{CO}_{2}$ partial pressure of $\approx 4 \times 10^{-4}$ bar ( $1 \mathrm{PAL}$ ) would extend above the magnetopause stand-off distance. If this expansion occurs, strong solar wind ion pick up loss rates (Lichtenegger et al. 2010; Scherf et al. 2018), in addition to the high thermal loss rates (Tian et al. 2008a, 2008b) take place. According to Lichtenegger et al. (2010) and Scherf et al. (2018), assuming a present-day atmospheric composition on early Earth, it would have led to a loss in $\mathrm{N}_{2}$ of $>3$ bar during less than $50 \mathrm{Myr}$.

In addition, according to Lichtenegger et al. (2010), the solar wind induced mass loss rate of $\mathrm{N}^{+}$for a 7 times higher EUV flux, exposed to a present-day atmosphere, would be about $9.36 \times 10^{6} \mathrm{~g} \mathrm{~s}^{-1}$. To compare this atmospheric mass loss rate with the outgassing rate from the surface via mantle wedges, one can use the empirical $\mathrm{N}_{2}$ flux which is released from the Central American volcanic arc system. At present, nitrogen is released from the mantle and discounting sedimentary input at the arc system with a rate of about $0.63 \times 10^{10} \mathrm{~mol}$ $\mathrm{yr}^{-1}$ (e.g., Fischer et al. 2002; Mikhail and Sverjensky 2014). If this outgassing rate were responsible for the build-up to the present atmospheric partial surface pressure within about 1.0 Gyr, one has to enhance the outgassing rate by about 20 times. In this way, one obtains a mass rate of $\mathrm{N}_{2}$ released into the atmosphere of about $5.65 \times 10^{4} \mathrm{~g} \mathrm{~s}^{-1}$. This rate is about 100 times lower than the modeled mass loss rate to space (Lichtenegger et al. 2010).

These results indicate that no dense $\mathrm{N}_{2}$ atmosphere could have been build up before about 3.5 Gyr ago, if the upper atmosphere had $\mathrm{CO}_{2}$ contents $<100 \mathrm{PAL}$, even if the young Sun were a slowly rotating young G-star. If the young Sun was a fast rotating young G-star with $\mathrm{CO}_{2}<100$ PAL then no dense $\mathrm{N}_{2}$ atmosphere could build up at ages of about $<3 \mathrm{Gyr}$ ago. However, from paleosol studies (Holland 1984; Berner and Kothvala 2001; Dauphas and Kasting 2011; Reinhard and Planavsky 2011; Goldblatt and Zahnle 2011) it is possible that the atmosphere contained $\mathrm{CO}_{2}$ values of $\approx 100-1000$ PAL during the late Hadean, and early Archean about 4.2-3.0 Gyr ago (Hessler et al. 2004). In addition, recent studies by Kanzaki and Murakami (2015) even indicate that $\approx 2.7$ Gyr ago the $\mathrm{CO}_{2}$ surface pressure was in the range of about 80 to several $100 \mathrm{PAL}$, decreasing gradually during the Archean 


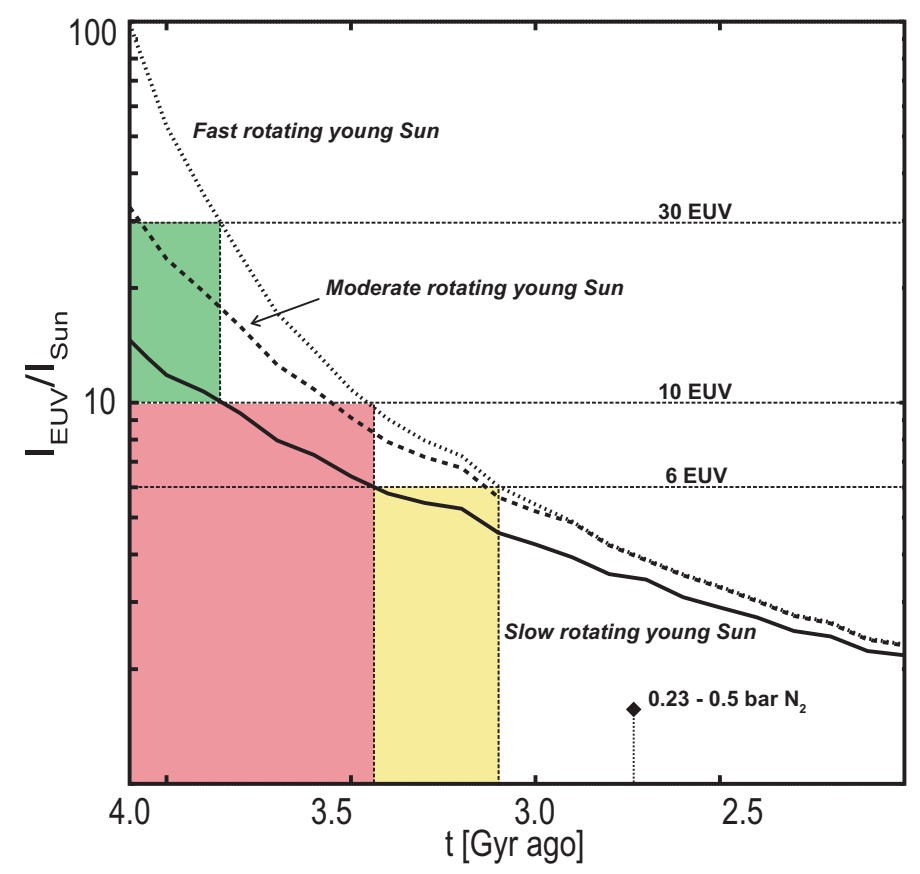

Fig. 18 Solar EUV flux enhancement factor according to a slow (solid line), moderate (dashed line) and fast (dotted line) rotating young star between about 2.0-4.1 Gyr ago (Tu et al. 2015). The green, red and yellow shaded areas mark the time periods, when the EUV fluxes for the two rotators decreased successively from $\times 30$ to $\times 10$ and to $\times 6$ times solar value. One of the horizontal dashed lines marks the 6 EUV level, the EUV flux enhancement where the outgassing of $\mathrm{N}_{2}$ molecules could start to build up an atmosphere that reached about 2.7 Gyr ago a surface pressure within about 0.23-0.5 bar (Marty et al. 2013; Som et al. 2016) if the young Sun was a slow rotator.

and early Paleoproterozoic. If the atmosphere had $\mathrm{CO}_{2}$ contents that have been 100-1000 times higher compared to that of today's value, the upper atmosphere would have been cooled via IR-emission caused by the $\mathrm{CO}_{2}$ molecules (e.g. Kulikov et al. 2006, 2007), and the exobase levels could be much closer to the planet (see Fig. 19) and escape rates could dramatically decrease. However, even a 1 bar atmosphere with $96 \% \mathrm{CO}_{2}$ would start to hydrodynamically expand under an EUV flux of $\approx 30$ times the present-day value, resulting in strong atmospheric escape rates (Kulikov et al. 2007; Scherf et al. 2018). Such high EUV fluxes would be reached by a fast rotating Sun at $\approx 3.85 \mathrm{Gyr}$ (see Fig. 18) and by a moderately rotating Sun at $\approx 4.1 \mathrm{Gyr}$, whereas a slowly rotating Sun reaches values below $30 \mathrm{EUV}$ already before $\approx 4.5 \mathrm{Gyr}$.

\subsection{Earth's ${ }^{14} \mathrm{~N} /{ }^{15} \mathrm{~N}$ isotope atmosphere-crust-mantle disequilibrium}

As illustrated in Fig. 15, from isotope studies related to mantle geodynamics and interaction processes with the atmosphere-crust-mantle system, it is known that Earth's upper, and deeper mantle which consist of primordial nitrogen, are depleted compared to the atmosphere in the heavier nitrogen isotope by $\delta^{15} \mathrm{~N} \approx-5 \%$, and $\delta^{15} \mathrm{~N} \approx-40 \%$, respectively (Cartigny and Marty 2013). According to Cartigny and Marty (2013) geochemical, geodynamical and geophysical 


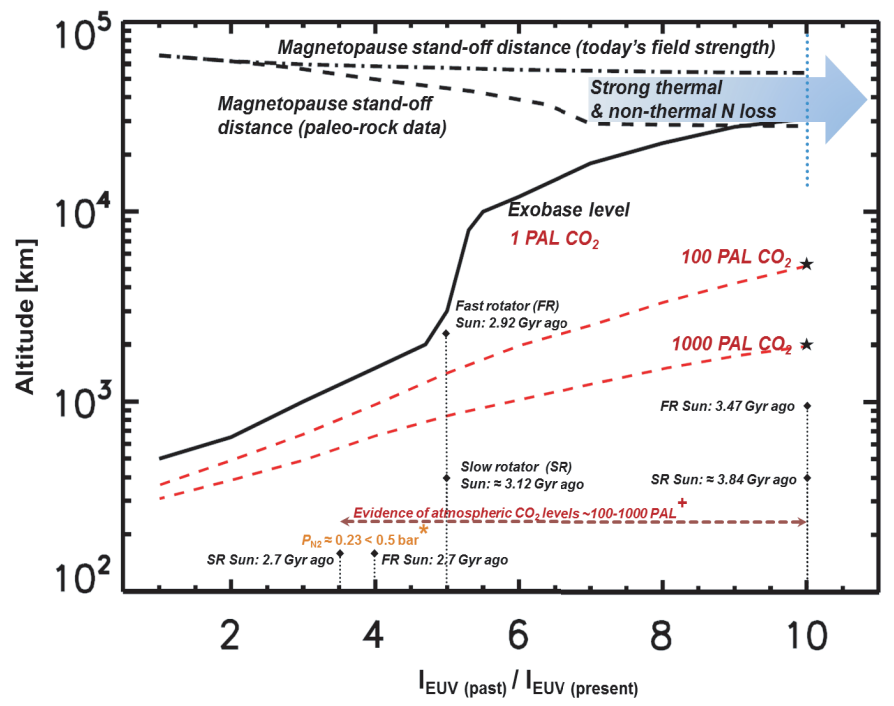

Fig. 19 Exobase levels for a nitrogen-dominated atmosphere with $1 \mathrm{PAL} \mathrm{CO}_{2}$ according to Tian et al. (2008a, 2008b) (solid line) and for 100 and $1000 \mathrm{PAL} \mathrm{CO}_{2}$ based on the studies of Tian et al. (2008a, 2008b) and Kulikov et al. (2006, 2007). Magnetopause stand-off distance according to paleorock data of Tarduno et al. (2015) (dashed line) and for today's magnetic moment (dashed-dotted line) as a function of solar EUV flux enhancement. The ages when the young Sun reached the 10 EUV values are marked for a slowly and fast rotating young Sun according to Tu et al. (2015). Atmospheric surface pressure values of about 0.23 bar (Som et al. 2016), at about 2.7 Gyr and corresponding EUV flux enhancements.

reasons can be most likely excluded for this nitrogen disequilibrium because one would then expect the mantle to be enriched in the heavier ${ }^{15} \mathrm{~N}$ isotope instead of the lighter ${ }^{14} \mathrm{~N}$ isotope.

Other more likely possibilities for the solution of these isotope discrepancies are preferential atmospheric nitrogen escape of ${ }^{14} \mathrm{~N}$ that resulted in an enrichment of ${ }^{15} \mathrm{~N}$ in the atmosphere/crust compared to the outgassed primordial nitrogen in the mantle, or heterogeneous accretion of the Earth, which would then need a subsequent contribution of chondritic material during the "late veneer" (Cartigny and Marty 2013). As discussed in previous sections the recent Ru isotopic analysis of chondritic material by Fischer-Gödde and Kleine (2017) points to an inner Solar System origin of the "late veneer" impactors and excludes now carbonaceous chondritic material. In addition, denitrification tends to enrich the atmosphere with the lighter isotope ${ }^{14} \mathrm{~N}$ (see below).

In light of these latest findings, one can expect that the solution of Earth's nitrogen isotope disequilibrium lies in an enrichment of ${ }^{15} \mathrm{~N}$ of primordial outgassed atmospheric nitrogen by solar wind ion pick up escape of an EUV heated extended upper atmosphere during the Archean eon. As illustrated in Fig. 20, according to this hypothesis the nitrogen isotope composition of the Earth's atmosphere/crust, which was enriched in the heavier ${ }^{15} \mathrm{~N}$ isotope compared to the primordial mantle nitrogen, could originate from mantle outgassing followed by solar wind induced ion pick up escape (Lichtenegger et al. 2010, Scherf et al. 2018) of an EUV heated extended upper atmosphere (see also Fig. 20). At the end of this escape phase, atmospheric nitrogen would have had a positive $\delta^{15} \mathrm{~N}$ value, compared to the negative value of the mantle. 
One should point out that the early nonthermal atmospheric escape process of the $\mathrm{N}_{2-}$ dominated atmosphere would mainly focus on nitrogen and not strongly affect other heavy minor species such as atmospheric $\mathrm{Ne}$ and other noble gases, whose isotope anomalies are caused by hydrodynamic drag of escaping hydrogen during the earliest stages of atmosphere evolution as discussed in Sect. 2. Moreover, early Earth's noble gas signatures could have been also modified by a few Kuiper-belt impactors during the late heavy bombardment, while Earth's nitrogen inventory was not affected (Marty and Meibaum 2007). In addition, the relationship between noble gas photoionization and organic photochemistry, as well as polar escape during the late Hadean/early Archean could have also preferentially fractionated Xe isotopes because of the higher EUV flux (Hébrard and Marty 2014), as discussed in subSect. 7.1.

The EUV flux decreased very fast from an EUV enhancement factor of 7 to 5 within about 0.15 Gyr (Tu et al. 2015). The EUV-driven atmosphere expansion decreases also to a great extent when the EUV flux decreased from 7 to 6 and 5 times compared to that of the present-day solar value. Depending on the amount of atmospheric $\mathrm{CO}_{2}$, one can expect that an EUV flux enhancement factor of $\geq 5-6$ corresponds to the time, when the nonthermal nitrogen escape weakened, ${ }^{15} \mathrm{~N}$ enrichment stopped, and outgassed $\mathrm{N}_{2}$ could start to build up a denser "secondary" atmosphere.

In the case that the young Sun was a slow rotator, and we assume low $\mathrm{CO}_{2}$ levels comparable to that of present Earth, the atmospheric $\mathrm{N}_{2}$ could start to build up at around $\approx 3.5$ Gyr when the EUV flux decreased to about 6 times the present-day value. The build-up depends mainly on the outgassing rate, the biological fixation, and weathering by lightning and combustion. By assuming a 20 times higher outgassing rate than at present, the same fixation and weathering rate together with no denitrification, such an atmosphere could reach about up to 0.27 bar by $\approx 2.7 \mathrm{Gyr}$, while for a fast rotator the loss rates are larger resulting in only $\approx 0.1$ bar $\mathrm{N}_{2}$ at $2.7 \mathrm{Gyr}$ ago. One should also note, that in the latter case, Earth's present $\mathrm{N}_{2}$ atmosphere/crust would have been most likely stronger enriched in ${ }^{15} \mathrm{~N}$ compared to the mantle nitrogen, broadly to be similar to Mars' atmosphere. By assuming higher $\mathrm{CO}_{2}$ values during the Archean than present-day, as strongly suggested by recent paleorock data analysis (e.g. Kanzaki and Murakami, 2015), the escape rate would have been lower and the atmosphere could have started to build up earlier. However, outgassing rates, fixation and weathering during the Archean are not clearly estimated. The ${ }^{14} \mathrm{~N} /{ }^{15} \mathrm{~N}$ disequilibrium can therefore give important insight into these early atmospheric escape processes, since it is indicating that escape of nitrogen certainly took place, even though higher $\mathrm{CO}_{2}$ abundances during the Archean most probably prevented strong escape of the atmosphere.

The secular trend in enriched sedimentary $\delta^{15} \mathrm{~N}$ during Earth's history indicates that denitrification started at about $2.7 \mathrm{Gyr}$ ago by heterotrophic bacteria (Farquhar et al. 2014; Zerkle et al. 2017). One should note that nitrogen fixation by anaerobic bacteria produce also small negative fractionations from atmospheric $\mathrm{N}_{2}$, that result in $\delta^{15} \mathrm{~N}$ values of $-4 \% 0-0 \%$, while denitrification preferentially releases the lighter $\delta^{14} \mathrm{~N}$ isotopes into the atmosphere in $\delta^{15} \mathrm{~N}$ of $10 \%$ to $25 \%$ (Zerkle et al. 2008, 2017). From this data one can also see that prior to $2.7 \mathrm{Gyr}$ ago the crust was enriched in ${ }^{15} \mathrm{~N}$ in the range of $-6 \%$ to $+13 \%$ (Farquhar et al. 2014). This data indicate that the atmospheric $\mathrm{N}_{2}$ during the early Archean should have been even more enriched. As one can see in Fig. 19, this period corresponds to a solar EUV flux that was higher than 5 times compared to that of today's solar value, where an $\mathrm{N}_{2}$-dominated upper atmosphere would have switched from hydrodynamic to hydrostatic conditions. Since the Archean and especially during and after the GOE, the atmosphere was modified by efficient outgassing of primordial nitrogen that is enriched in the lighter ${ }^{14} \mathrm{~N}$ isotopes via mid-ocean ridge volcanos, because of plate tectonics and oxidized mantle wedge conditions (Mikhail 


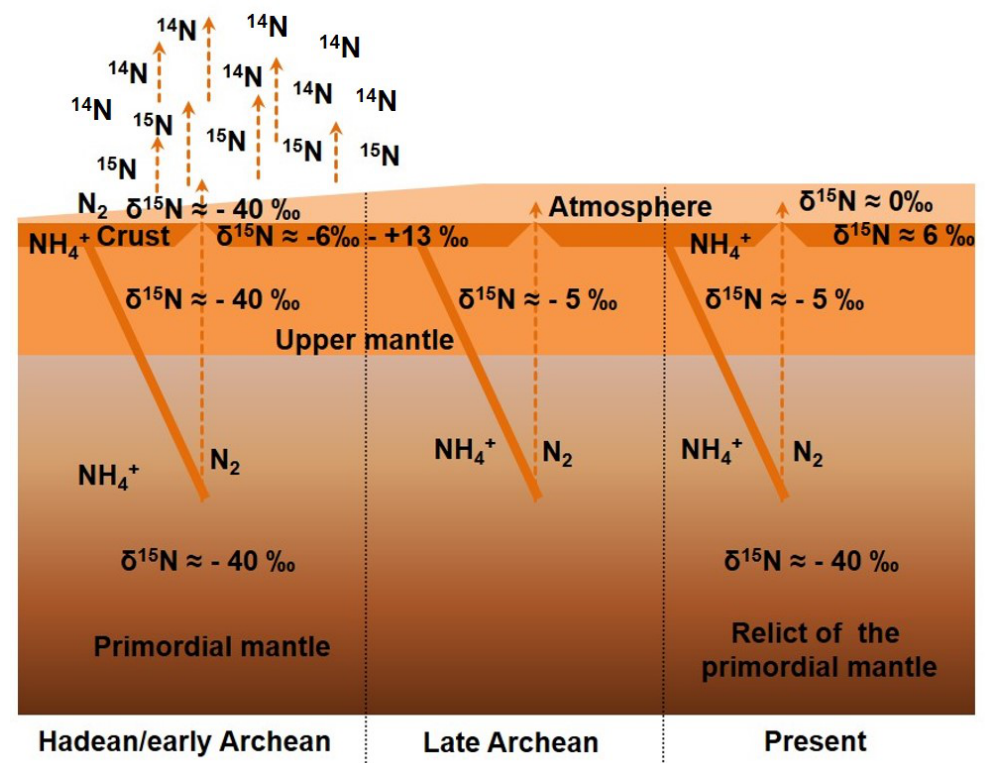

Fig. 20 Illustration of the most likely origin of Earth's so-called nitrogen isotope disequilibrium during Earth's history as described in the main text between the deeper mantle, the surface reservoirs and the atmosphere.

and Sverjenski 2014; see also Fig. 17). Therefore, mantle wedges beneath arc volcanoes on Earth may have not only acted as a "nitrogen-pump" out of Earth's mantle, which resulted not only in an $\mathrm{N}_{2}$ enrichment relative to the primordial noble gases (Mikhail and Sverjenski 2014; Wordsworth 2016a; Zerkle and Mikhail 2017), but also to an enrichment in lighter ${ }^{14} \mathrm{~N}$ isotopes over time. The light enrichment in the present day crust in the heavier ${ }^{15} \mathrm{~N}$ isotope by $\delta^{15} \mathrm{~N} \approx 6 \%$ compared to the atmosphere most likely evolved to a saturated value related to geological and biological processes.

\section{Earth's atmosphere at the time of life's origin}

It is commonly expected that frequent large asteroid impacts produced globally lethal environmental conditions by ocean water evaporation over large scales during the Hadean (e.g., Maher and Stevenson 1988; Sleep et al. 1989). Maher and Stevenson (1988) suggest that at the end of the Hadean deep marine hydrothermal settings would have offered suitable environments for the origin of life which could have started $\approx 4.0-4.2 \mathrm{Gyr}$ ago, while life close to or on the surface could have originated $\approx 3.7-4.2 \mathrm{Gyr}$ ago (e.g., Hedges 2002). This timeline agrees with the recent discovery of fossilized microorganisms suggesting that life existed already 3.8 Gyr and up to 4.2 Gyr ago in Earth's oldest submarine-hydrothermal vents at the Nuvvuagittuq belt in Quebec, Canada (Dodd et al. 2017) and in the Isua supracrustal belt metacarbonate rocks (Nutman et al. 2016). 
7.1 Production of prebiotic molecules and additional greenhouse gases besides $\mathrm{CO}_{2}$

According to Marty and Meibom (2007), the amounts of water and $\mathrm{N}_{2}$ delivered during the Late Heavy Bombardment (LHB) $\approx 3.8$ - 4.1 Gyr ago, should have been very small, compared to the inventories in the mantle and oceans. However, some studies indicate that organics of variable complexity, in amounts comparable to the mass of today's biosphere $\left(\approx 1.15 \times 10^{19} \mathrm{~g}\right)$, could have been delivered by impactors, which originated in the outer Solar System between the later Hadean and the LHB (Anders 1989; Bockelée-Morvan et al. 2004; Marty and Meibom 2007). Impactors during the late Hadean, and early Archean might have also provided the energy and created the redox conditions necessary to synthesize complex organics (e.g., Marty and Meibom 2007; Brack et al. 2010).

Airapetian et al. (2016) studied the prebiotic chemistry and greenhouse warming of the early Earth by including solar activity effects (i.e., superflares, frequent collisions with Coronal Mass Ejections (CME), solar wind induced shocks, high energy particles and compressed magnetosphere) caused by the active young Sun. These authors constrained their magnetohydrodynamic simulations using data obtained from the Kepler Space Telescope. They found that if early Earth had an $\mathrm{N}_{2}$-dominated atmosphere (80\%), and greenhouse gases such as $\mathrm{CO}_{2}(20 \%)$ and $\mathrm{CH}_{4}(0.03 \%)$ (Cnossen et al. 2007), under the assumed solar environmental conditions the polar cap openings of the magnetosphere would have provided pathways for high energy particles. According to Airapetian et al. (2016), these particles would then penetrate into the atmosphere, affecting the atmospheric chemistry, so that the majority of the $\mathrm{CO}_{2}$ and $\mathrm{CH}_{4}$ are photochemically destroyed and a large amount of the greenhouse gas nitrous oxide $\left(\mathrm{N}_{2} \mathrm{O}\right)$ is produced. However, it should be noted that this study does not address the fact that the $\mathrm{N}_{2} \mathrm{O}$, which is produced high in the atmosphere will most likely be photolyzed before it could ever make it down to the troposphere.

By neglecting the possible photochemical destruction of $\mathrm{N}_{2} \mathrm{O}$ in the upper atmosphere, these authors found that other reactions in their chemical model, which produce $\mathrm{CH}, \mathrm{NH}$ and $\mathrm{NO}$, set the stage for the formation of $\mathrm{N}_{2} \mathrm{O}$ and $\mathrm{HCN}$ and other $\mathrm{N}$-containing species. The concentration of $\mathrm{HCN}$ reaches up to about tens of parts per million in the lower atmosphere. According to Airapetian et al. (2016), these molecules, which have been relevant for the formation of life on Earth (i.e. HCN polymerization, known to produce various amino acids, the building blocks of proteins (Miyakawy et al. 2002)), rain out into the surface water reservoirs, where complex life may have formed and will participate in higher order organic chemistry.

What remains unclear is, if $\mathrm{N}_{2}$ really was the dominant atmospheric species at that time (see also sub-Sect. 6.2)? As pointed out earlier, from the recent analysis of paleorock data by Kanzaki and Murakami (2015) and the low partial surface pressure values obtained from paleorock studies (Marty et al. 2013; Som et al. 2016) one can expect that $\mathrm{N}_{2}$ was less abundant than $\mathrm{CO}_{2}$ during the early Archean. Furthermore, to which extent $\mathrm{N}_{2} \mathrm{O}$ is destroyed by photochemical processes in the upper atmosphere needs to be addressed by coupled climate-photochemical models, which have to incorporate this additional source of $\mathrm{N}_{2} \mathrm{O}$ into their modeling.

As illustrated in Fig. 22, a combination of the above described reactions and the delivery of meteoritic organic material, as evidenced by the presence of carbonaceous components in meteorites (e.g., Maurette and Brack 2006; Brack et al. 2010), around the LHB period (e.g., Bottke et al. 2012) most likely played an initial important role in the availability of the building blocks of life during the late Hadean and early Archean.

Hydrothermal systems are considered to be likely environments for the synthesis of prebiotic organic molecules and even for primitive life (e.g, Holm 1992; Brack et al. 2010). 


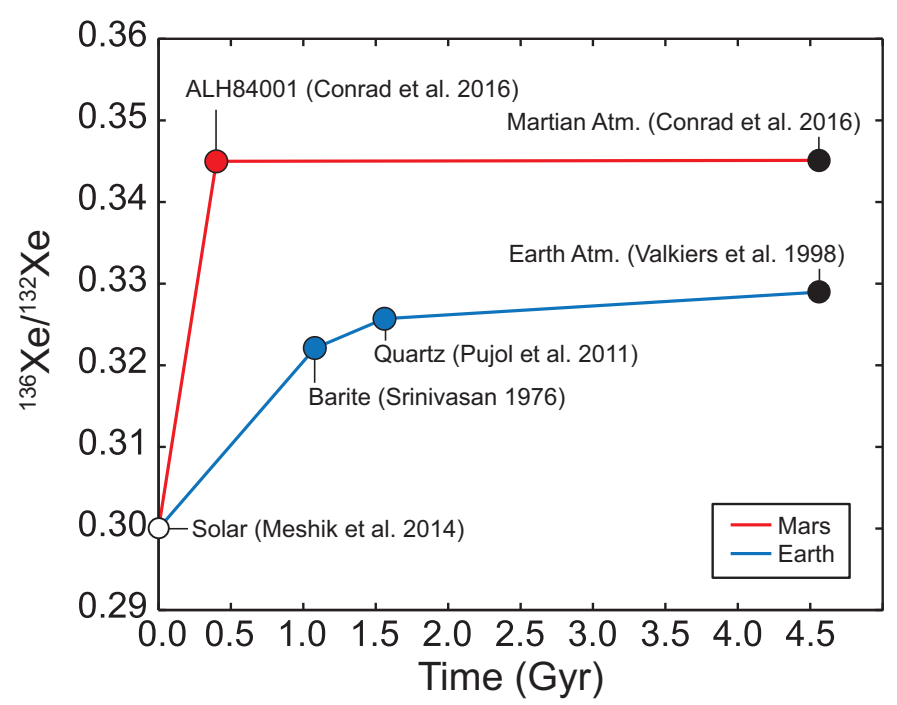

Fig. 21 Terrestrial and Martian ${ }^{136} \mathrm{Xe} /{ }^{132} \mathrm{Xe}$ ratios as a function of time after Cassata (2017). Data sources as described in the Figure.

Starting from these building blocks and given suitable environmental conditions suggests that a chemical evolution of the precursors of life to the earliest life-forms, such as methanogens, most likely occurred during the late Hadean (Hedges 2002; Nutman et al. 2016; Dodd et al. 2017). Methanogenesis is the production of $\mathrm{CH}_{4}$ by the earliest microbes known as methanogens from $\mathrm{H}_{2}$ and $\mathrm{CO}_{2}$ (e.g., Thauer 1998; Brack et al. 2010; Catling et al. 2001).

However, organic hazes played also an important role in the fractionation of Xe noble gases. As briefly mentioned above, chondritic-like Xe trapped in Archean rocks indicate that Earth's atmospheric Xe was fractionated before $\approx 2.5$ Gyr ago (Srinivasan 1976; Pujol et al. 2009; Pujol et al. 2011). Hébrard and Marty (2014) found that the interaction between the organic haze and ionized Xe resulted in Xe-trapping into organics, which is more efficient compared to other noble gases. Hébrard and Marty (2014) suggest that this trapping resulted in a significant enrichment of heavier Xe isotopes relative to the lighter ones. It is therefore expected that on Earth Xe isotopes experienced this photochemical fractionation during the Archean period, while another fraction should have escaped via polar outflow in a hydrogenrich upper atmosphere, which originated from dissociation of $\mathrm{CH}_{4}$ to space. The partly ionized hydrogen can drag away the heavy $\mathrm{Xe}^{+}$ions along the open magnetic fiel lines due to the strong Coulomb interaction between these ions (Zahnle 2015; Catling and Kasting 2017). This combination, i.e. Xe trapped in haze and escape to space, most likely explains the evolution and enrichment of Xe isotopes observed in Archean barite (Srinivasan 1976; Pujol et al. 2009; Pujol et al. 2011) over time, as can be seen in Fig. 21.

As mentioned above, studies of nitrogen and Ar isotopes in fluid inclusions trapped in 3.0-3.5 Gyr old hydrothermal quartz indicate that the partial pressure of $\mathrm{N}_{2}$ was at that age between 0.5-1.1 bar (Marty et al. 2013) and possibly $\leq 0.23$ bar $\sim 2.7$ Gyr ago (Som et al. 2016). These low surface pressure values contradict theories such as $\mathrm{N}_{2}$-enhanced greenhouse warming in the Archean, which could have been caused by pressure broadening if the atmosphere had an $\mathrm{N}_{2}$ partial surface pressure of about 1.6 - 2.4 bar, $2.5 \mathrm{Gyr}$ ago (Goldblatt et al. 2009). The paleorock studies of Marty et al. (2013) and Som et al. (2016) also contradict hypotheses that above mentioned biological nitrogen fixation has caused 


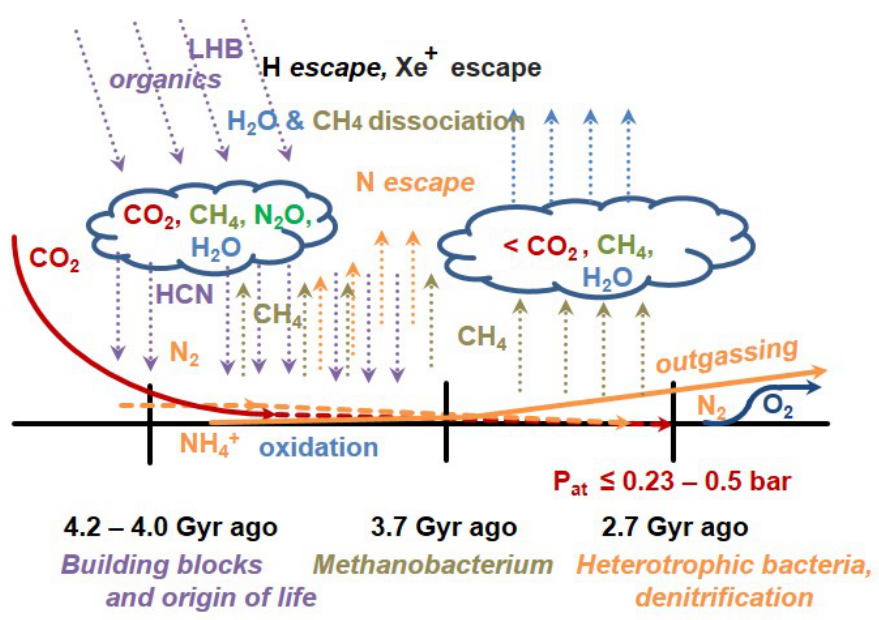

Fig. 22 Atmosphere constituents during the time when the building blocks for life accumulated in the ocean due to meteoritic impacts and/or formed in the atmosphere via energetic particle events, photochemistry and lightning. At about 3.7 - 4.0 Gyr ago methanogenic life originated and the greenhouse gas $\mathrm{CH}_{4}$ was also released into the atmosphere. The atmospheric chemistry in the $\mathrm{CH}_{4}$-rich early atmosphere resulted in an increased production of organic haze, where a fraction of lighter Xe isotopes was trapped, while another fraction escaped from the atmosphere to space without isotope selection.

atmospheric nitrogen levels to decrease over geological time which could have led to the before mentioned higher atmospheric surface pressures.

In order to keep the surface of early Earth above the freezing point of water different greenhouse gases have been proposed such as $\mathrm{CH}_{4}$, (e.g. Kiehl and Dickinson 1987; HaqqMisra et al. 2008), which additionally to $\mathrm{N}_{2} \mathrm{O}$ (e.g. Buick 2007; Grenfell et al. 2011; Roberson et al. 2011, Airapetian et al. 2016), some levels of $\mathrm{CO}_{2}$ (e.g., Rye et al. 1995; Hessler et al. 2004; Kasting and Ono 2006; Sheldon 2006; Haqq-Misra et al. 2008; von Paris et al. 2008; Driese et al. 2011; Kanzaki and Murakami 2015), and possible effects related to the albedo (Rosing et al. 2010a), kept early Earth's surface temperature most likely above zero during the early Archean FYSP period. Note however, the $\mathrm{CO}_{2}$ estimate by Rosing et al. 2010a has been critized by several authors such as Dauphas and Kasting (2011), Reinhard and Planavsky (2011), and Goldblatt and Zahnle (2011). Applying advanced 3D models including atmosphere-ocean coupling and including the effect of clouds result in climate simulations which could successfully address the FYSP. Other potential solutions have been suggested by several studies of the early Earth, see e.g. Charnay et al. (2013; 2017), Wolf and Toon (2013), Kunze et al. (2014). The results of these studies indicate that even for limited amounts of $\mathrm{CO}_{2}$ and $\mathrm{CH}_{4}$, which are in agreement with the geological record for that time, the early Earth may have been partially ice free, with liquid water in the equatorial region despite global mean temperatures below the freezing point of water. For a lower surface pressure, one also expects a higher volume mixing ratio of $\mathrm{H}_{2} \mathrm{O}$ vapor. However, a lower surface pressure would have exacerbated the FYSP due to less atmospheric mass hence warming by greenhouse gases. 


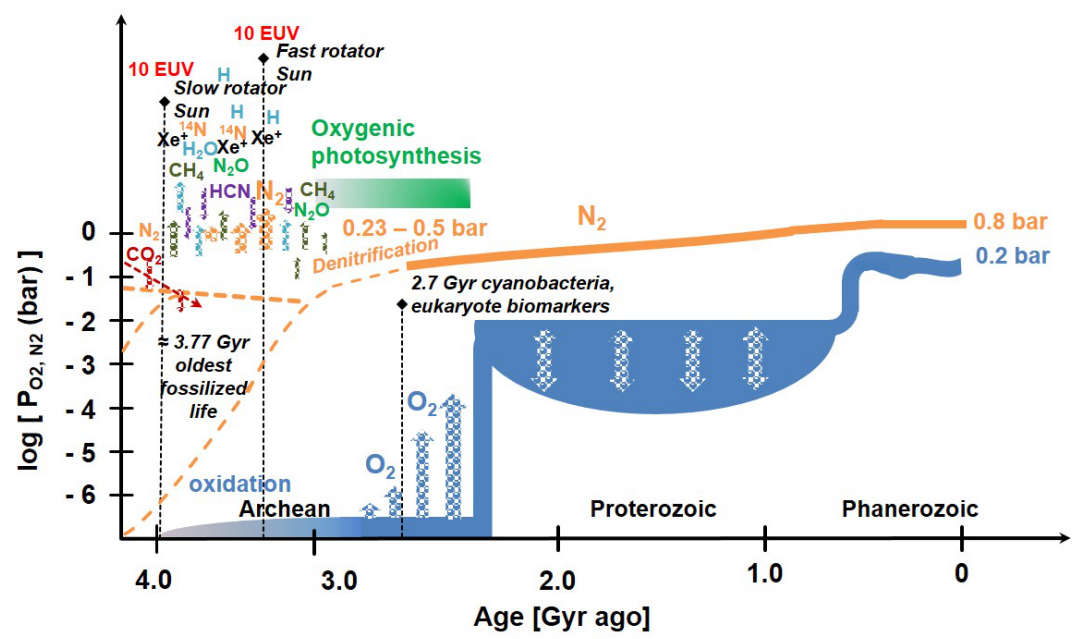

Fig. 23 Illustration of the surface partial pressure build up of early Earth's $\mathrm{N}_{2}$ and $\mathrm{O}_{2}$ atmosphere since the late Hadean. During the early Archean the atmospheric pressure was low and at about 3.5 Gyr ago $\mathrm{N}_{2}$ was again efficiently outgassed via mantle wedge volcanos so that a surface pressure of about $0.23-0.5$ bar was reached about 2.7 Gyr ago.

7.2 The great oxidation event and the rise of atmospheric surface pressure

As pointed out in studies by Catling et al. (2001) and Zahnle et al. (2006) the $\mathrm{CH}_{4}$ content in early Earth's atmosphere implies a strong hydrogen escape to space (Catling et al. 2001; Catling and Claire 2005; Tian et al. 2005; Erkaev et al. 2013), because of the higher EUV flux of the young Sun. Such high hydrogen losses related to the photodissociation of $\mathrm{CH}_{4}$ and $\mathrm{H}_{2} \mathrm{O}$ will then lead to oxidation of the crust (e.g. Catling et al. 2001, 2005; Catling and Claire 2005; Zahnle et al. 2006). Furthermore, as was shown by Wordsworth and Pierrehumbert (2014), atmospheres with low $\mathrm{N}_{2}$ amounts have problems to trap $\mathrm{H}_{2} \mathrm{O}$ and hence can oxidize via hydrogen escape. This processes can even lead to the build up of abiotic $\mathrm{O}_{2}$-dominated atmospheres (Wordsworth and Pierrehumbert 2014). After the crust and upper mantle have been oxidized $\mathrm{N}_{2}$ is released efficiently due to the "redox pump" process (Mikhail and Sverjensky 2014; Wordsworth 2016a; Zerkle and Mikhail 2017).

Fig. 23 illustrates the general picture of the build up of early Earth's $\mathrm{N}_{2}$ and $\mathrm{O}_{2}$ atmospheres from about 4.0 Gyr ago up until the present. Earth's early atmosphere likely had virtually no free $\mathrm{O}_{2}$ in its lower atmosphere before photosynthesis developed, whereas the upper atmosphere could have been oxidized due to stratospheric $\mathrm{O}_{2}$ production via $\mathrm{CO}_{2}$ photolysis (see e.g. Kasting and Donahue 1980; Kasting et al. 1984a; Catling and Claire 2005; Zahnle et al. 2006; Domagal-Goldman et al. 2014; Gebauer et al. 2017). The rise of atmospheric $\mathrm{O}_{2}(\mathrm{GOE})$ took place at $\approx 2.2$ - 2.4 Gyr ago (e.g. Catling et al. 2001; Kopp et al. 2005; Zahnle et al. 2006; Gaillard et al. 2011; Lyons et al. 2014; Luo et al. 2016) and there are several proposed mechanisms that might have triggered the GOE. According to Zahnle et al. (2006) the GOE was most likely a consequence of a collapse in atmospheric $\mathrm{CH}_{4}$ levels accompanied by a reduced greenhouse effect that resulted in the first major glaciation in Earth's history. The decrease in $\mathrm{CH}_{4}$ would have resulted in an increase of $\mathrm{O}_{2}$ via missing chemical sinks of $\mathrm{O}_{2}$, that can be seen as an alternative to previously proposed scenarios, where the rise in atmospheric $\mathrm{O}_{2}$ is proposed to have caused the collapse of $\mathrm{CH}_{4}$ due to its 
lethal effect on methanogens (e.g. Kopp et al. 2005). However, Goldblatt (2008) and Gebauer et al. (2017) calculated an increase in $\mathrm{CH}_{4}$ during the GOE since in their calculations an increase in atmospheric $\mathrm{O}_{2}$ leads to an increase in atmospheric $\mathrm{O}_{3}$. This results in enhanced shielding from $\mathrm{UV}$ (hence $\mathrm{OH}$ ) which are major sinks for $\mathrm{CH}_{4}$. Whatever scenario occurred, because $\mathrm{CH}_{4}$ and $\mathrm{O}_{2}$ react strongly, the rise of $\mathrm{O}_{2}$ and the decrease of $\mathrm{CH}_{4}$ have certainly been linked. For a more comprehensive discussion about possible mechanisms triggering the GOE we refer to Kasting (2013).

However, the geological record cannot be used for directly constraining the atmospheric $\mathrm{CH}_{4}$ levels during the Proterozoic. Olson et al. (2016) quantified $\mathrm{CH}_{4}$ fluxes from the marine biosphere to examine the capacity of biogenic $\mathrm{CH}_{4}$ to compensate for the FYSP during the years before the emergence of metazoan life. The results of their study demonstrate that anaerobic oxidation of $\mathrm{CH}_{4}$ coupled to $\mathrm{SO}_{4}^{2-}$ reduction is the strongest obstacle for atmospheric $\mathrm{CH}_{4}$ build up with an upper value for its atmospheric abundance of $\approx 0.001 \%$ for the second half of Earth's history regardless of atmospheric oxygen partial pressure. This value is about 30 times lower than the $\mathrm{CH}_{4}$ contents assumed in the above mentioned study of Airapetian et al. (2016).

Most likely (as illustrated in Figs. 22 and 23) after life originated, the combination of a mixture of greenhouse gases $\left(\mathrm{CO}_{2}, \mathrm{CH}_{4}, \mathrm{~N}_{2} \mathrm{O}\right)$ kept early Earth's surface above freezing until the GOE and its related global glaciation event (e.g., Feulner 2012; Charey et al. 2013; Wolf and Toon 2013). As pointed out by Zerkle and Mikhail (2017) and Zerkle et al. (2017) one can expect that the flux of $\mathrm{N}_{2}$ into the atmosphere has dramatically shifted during the GOE, because the oxidizing potential of the subducted material such as oceanic sediments and the altered oceanic crust must have been different. Ingassing should have dominated preGOE and outgassing post-GOE periods. According to Zerkle and Mikhail (2017) following the GOE, subduction zones excessively outgassed $\mathrm{N}_{2}$ to the atmosphere, which over time resulted in Earth's higher $\mathrm{N}_{2} /{ }^{36} \mathrm{Ar}$ and $\mathrm{N}_{2} /{ }^{20} \mathrm{Ne}$ ratios compared to that of Venus and Mars (Mikhail and Sverensky 2014).

During the Proterozoic and Phanerozoic periods the $\mathrm{O}_{2}$ pressure most likely fluctuated (i.e., the series of snowball Earth events, further development and changes related to life, climatological changes, etc.). After the GOE Earth's ozone layer formed in the stratosphere and protected life forms against UVC radiation (Catling et al. 2005; Gebauer et al. 2017). After a second rise of $\mathrm{O}_{2}$ pressure at the beginning of the Phanerozoic period, plants and animal life prospered, which led to the $\mathrm{N}_{2}$ and $\mathrm{O}_{2}$ atmospheric partial pressures aside from some short-term fluctuations at about the same level as today (e.g., Catling and Claire 2005; Catling et al. 2005; Lyons et al. 2014). The present atmospheric $\mathrm{N}_{2}$ partial pressure has mostly remained at that because of nitrogen's biological connection due to denitrification.

\subsection{Earth's atmosphere evolution since it's origin}

Earth's atmospheric evolution since the planet's origin is illustrated in Fig. 24. After a thin nebula-based $\mathrm{H}_{2}$-envelope was lost to space, due to the young Sun's EUV driven hydrodynamic escape, during a few Myrs after the nebular evaporated, carbonaceous chondritic (CC) material was added until proto-Earth accreted to its final mass after the Moon forming impact. Earth's last global magma ocean solidified and a steam atmosphere outgassed catastrophically. After the water vapor condensed (Hamano et al. 2013; Lebrun et al. 2013; Massol et al. 2016; Salvador et al. 2017) Earth's earliest ocean originated and atmospheric $\mathrm{CO}_{2}$ weathered into the surface and formed carbonates (see Sect. 4). Atmospheric nitrogen most likely weathered into the surface during the hot magmatic surface environment and/or 


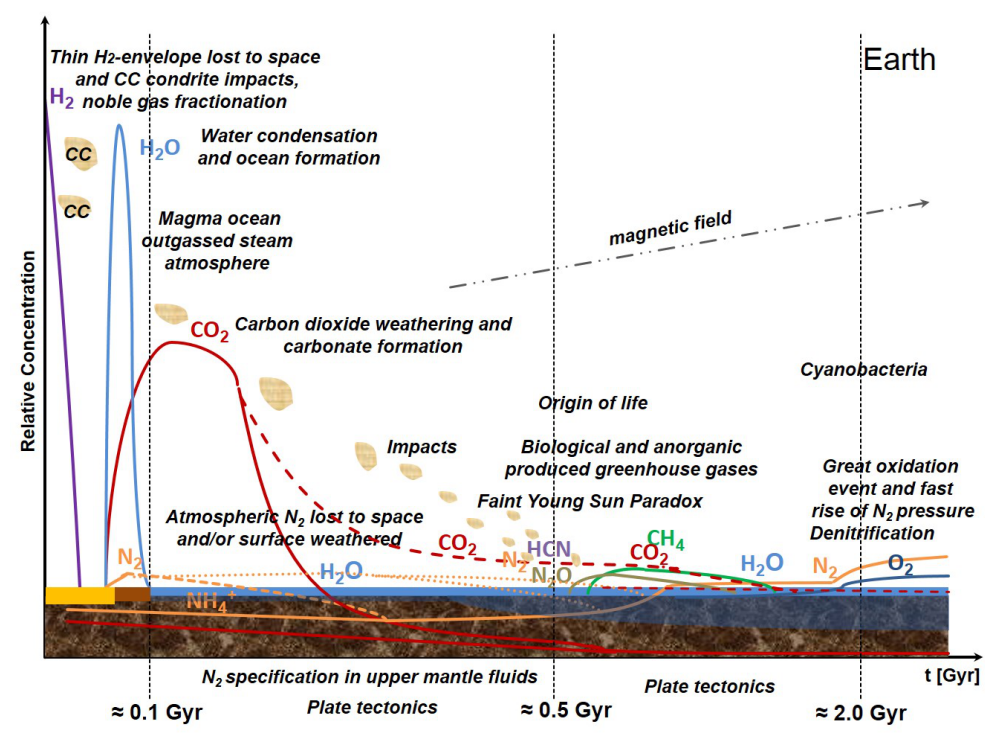

Fig. 24 Illustration of Earth's atmosphere evolution since its origin. Volatiles were delivered early during the main stages of the planet's formation from the outer Solar System via impacting carbonaceous chondrites (CC) and a large fraction of water could enter the mantle. After a thin nebula-based $\mathrm{H}_{2}$-envelope was lost to space and the Moon formed, Earth's large deep global magma ocean solidified and catastrophically outgassed a steam atmosphere. Water condensed and formed the earliest ocean on Earth's surface. Because of the liquid water, atmospheric $\mathrm{CO}_{2}$ was weathered from the atmosphere and transformed into carbonates. Most likely, because of the right temperature in the mantle and liquid water in the planet's interior a primitive form of plate tectonics evolved. A fraction of the $\mathrm{CO}_{2}$ remained in the atmosphere as a greenhouse gas and thermospheric IR-cooler until the Archean. As soon as Earth's crust and upper mantle was oxidized at $\approx 3.5-4.0$ Gyr ago $\mathrm{N}_{2}$ was again released via volcanos efficiently. After the origin of life, depending on the surface pressure and albedo, greenhouse gases such as $\mathrm{CO}_{2}, \mathrm{CH}_{4}, \mathrm{~N}_{2} \mathrm{O}$ kept early Earth's surface above freezing. Finally the oxidation stage of the early Earth was enhanced, $\mathrm{O}_{2}$ became an atmospheric species and because of denitrification caused by life forms, the $\mathrm{N}_{2}$ surface pressure raised finally to the present level.

were lost into the ocean/surface via nitrogen fixation processes (i.e., lightning, meteoritic impact heating) (Wordsworth 2016a; see also Sect. 5). In the subsurface it remained to a great extent together with a possibly not previously degassed nitrogen fraction in the form of $\mathrm{NH}_{4}^{+}$in aqueous upper mantle fluids as long as no efficient oxidization took place. The oxidation conditions during the Hadean and early Archean have been a direct consequence of plate tectonics-related subduction that injected oxidizing fluids into the upper mantle, as well as atmospheric escape of hydrogen from dissociated $\mathrm{H}_{2} \mathrm{O}$ molecules that left oxygen behind (e.g. Kasting et al. 1993a; Kelley and Cottrell 2009; Mikhail and Sverjensky 2014; Wordsworth 2016a). According to Kasting et al. (1993b), data of the redox state of chromite inclusions in diamonds and sulfide indicate that the mantle oxidation process was slow, so that reduced conditions could have prevailed until about 2.5 - 4.0 Gyr ago. This is further supported by recent studies of Aulbach and Stagno (2016) and Nicklas et al (2016) whereas e.g. Delano (2001), Canil (2002), Li and Lee (2004), Nicklas et al (2018) suggest only minor changes in the mantle oxygen fugacity since the early Archean, indicating that the mantle had reached its nearly-present day redox state by at least $3.5 \mathrm{Ga}$ ago. As soon as the upper mantle was oxidized to a great extent nitrogen could efficiently be degassed via the mantle wedges at convergent plate margins in the form of $\mathrm{N}_{2}$. As pointed out by Mikhail and Sverjensky 
(2014) and discussed above, the mantle wedges beneath arc volcanoes on Earth most likely have acted as a kind of " $\mathrm{N}_{2}$-pump" which released $\mathrm{N}_{2}$ out of Earth's uppermost mantle. After the origin of life, life forms modified and influenced Earth's atmosphere to a great extent (see Sects. 6 and 7).

\section{The makings of Earth-like habitats}

From this review, one can see that there were at least 3 major periods in Earth's evolution, which have been essential for the development of a class I habitat, as described in Lammer et al. (2009). These periods can be summarized as follows:

- The planet should not accrete protoplanet masses that are $>0.8 M_{\text {Earth }}$ as long as the nebular gas is not evaporated. More massive protoplanets can accumulate a huge amount of nebula gas, which can not be lost during the planet's life time (Lammer et al. 2014; Johnstone et al. 2015a; Owen and Mohanty 2016).

- During the second important period in a habitable planet's history (besides the magma ocean outgassed volatile-rich atmosphere), is the additional delivery of enough water and nitrogen-related gases from beyond the system's ice-line (e.g. Marty 2012). Moreover, planets in the habitable zone should have the right amount of heat producing radioactive materials (i.e., U, Th, K, etc.) and water, which set the initial conditions for the thermal evolution of the mantle and later onset of plate tectonics and magnetic dynamos during several Gyr (Murthy et al. 2003; O’Neill et al. 2007; O'Neill and Palme 2008; Jellinek and Jakson 2015; Noack and Breuer 2014).

- The third period relates to the operation of plate tectonics (e.g., Mikhail and Sverjensky 2014; Zerkle and Mikhail 2017) with its related atmosphere-surface interaction processes, as well as mantle-wedge volcanic outgassing (i.e., $\mathrm{N}_{2}$, etc.). This is very important for the evolution of Earth-like habitats. The active intrinsic magnetic dynamo (e.g., Murthy et al. 2003; O'Neill et al. 2007; Jellinek and Jakson 2015), which is also linked to plate tectonics, was/is important for the protection of surface life against high energy particles and should have played a role against large nonthermal escape rates of nitrogen caused by the high EUV flux of young stars. If a planet's atmosphere evolves an $\mathrm{N}_{2}$-dominated atmosphere with low $\mathrm{CO}_{2}$ levels too early in its history, then, no dense atmosphere will build up because of high atmospheric escape rates. The same efficient atmosphere losses occur when a planet evolves to an $\mathrm{N}_{2}$ atmosphere within the habitable zone of a dwarf star that remains active in X-rays and EUV over much longer time compared to solar-like G-type stars.

\section{Conclusions}

The most likely atmospheric formation and evolution scenarios of Venus, Earth and Mars since the origin of the Solar System $\approx 4.567$ Gyr ago have been discussed. The observed nonradiogenic atmospheric noble gas isotope fractionation of ${ }^{20} \mathrm{~N} /{ }^{22} \mathrm{Ne}$ and ${ }^{36} \mathrm{Ar} /{ }^{38} \mathrm{Ar}$ in the atmospheres of present Venus and Earth are in agreement with the "Grand Tack" hypothesis and measured Hf-W data, i.e. that these two planets accreted masses of $\approx \mathbf{0 . 5} \mathbf{- 0 . 7 5} M_{\text {Earth }}$ when the nebular gas in the disk was still present. During that time the planetary interior interacted with nebular gas due to the atmosphere interaction with magma oceans. After the nebula evaporated, both proto-planets were surrounded by thin $\mathrm{H}_{2}$-envelopes that were lost during the X-ray and EUV saturation phase of the young Sun by hydrodynamic escape and 
big impactors (i.e., Moon-forming event). On Earth the isotope signatures of $\mathrm{H}, \mathrm{N}, \mathrm{Ne}$ and $\mathrm{Ar}$ can be explained by mixing between two end members of solar and chondritic compositions. This indicates that volatiles such as $\mathrm{H}_{2} \mathrm{O}, \mathrm{CO}_{2}, \mathrm{NH}_{3}, \mathrm{HCN}$, etc. were delivered by chondritic impactors that are geologically undifferentiated meteorites from beyond the ice line during the main stages of planet formation. Larger planetary embryos which developed magma oceans, most likely lost the main fraction of volatiles due to hydrodynamic escape, in the inner Solar System after their magma oceans solidified.

On early Venus, after the nebular-based $\mathrm{H}_{2}$-envelope escaped, outgassed volatiles remained in the atmosphere. Water vapor most likely never reached the condensation point on early Venus and was lost early. $\mathrm{H}_{2} \mathrm{O}$ molecules dissociated and hydrogen escaped to space. A huge amount of oxygen that was left behind as a dissociation product of water from escaping hydrogen was mainly integrated into the hot and magmatic surface and oxidized the crust and the upper mantle. The loss of a suggested runaway greenhouse related water ocean to space, and the weathering of accumulated left behind oxygen in the absence of a deep magma ocean would be much more difficult, if atmospheric $\mathrm{H}_{2} \mathrm{O}$ condensed and a liquid surface ocean formed after the solar EUV flux decreased to lower values $>100 \mathrm{Myr}$ after the planet's origin. $\mathrm{CO}_{2}$ and $\mathrm{N}_{2}$ that was not outgassed after the last magma ocean solidified, could have been also released in Venus' atmosphere stepwise via super volcanos or resurfacing events from the interior since $\approx 4.4-4.5 \mathrm{Gyr}$ ago. Because of the lack of liquid surface oceans and plate tectonics, $\mathrm{CO}_{2}$ could not be weathered out of the atmosphere.

Early Earth's atmosphere evolution started in a similar way as early Venus, but the initially outgassed and impact delivered $\mathrm{H}_{2}$ could condense in $1 \mathrm{AU}$ and formed a liquid ocean on the planet's surface soon after the Moon-forming event. Water could enter the crust and upper mantle during or after the Moon forming impact and the thermal evolution of Earth's mantle evolved to conditions that a form of proto-plate tectonics and later during the Archean modern plate tectonics could evolve. The $\mathrm{CO}_{2}$ has been weathered from the atmosphere into the early ocean and transformed into carbonates until the late Hadean. A small fraction of the $\mathrm{CO}_{2}$ remained in the atmosphere as the main greenhouse gas until the Archean. Earth's atmospheric $\mathrm{N}_{2}$ that was outgassed/delivered when the last magma ocean solidified, was either partly lost to space, remained to some extent in the atmosphere and/or was weathered into the surface, where most of it remained stored in the form of $\mathrm{NH}_{4}^{+}$.

At the early Archean Earth's atmosphere was most likely dominated by $\mathrm{CO}_{2}$, but inferred from paleorock data, with partial surface pressure values of $<0.5$ bar. As soon as Earth's crust and upper mantle were oxidized $\approx 3.5-4.0$ Gyr ago, $\mathrm{N}_{2}$ was efficiently released via mantle wedge volcanos. Finally atmospheric oxygen was enhanced at the GOE, Earth's surface pressure raised from $\approx 0.23-0.5$ bar $\approx 2.7 \mathrm{Gyr}$ ago to higher values. More complex life forms originated and atmospheric $\mathrm{O}_{2}$ and $\mathrm{N}_{2}$ reached the present partial surface pressure values. Atmospheric $\mathrm{N}_{2}$ is massively affected by biological activity via biological fixation and also denitrification, which mainly keeps the atmospheric surface pressure at around the 1 bar value. Without modern plate tectonics and an efficient return flux into the atmosphere via denitrification and anaerobic ammonium oxidation, Earth's present day $\mathrm{N}_{2}$ atmosphere may be sequestered entirely into the surface within less than $100 \mathrm{Myr}$. Since early Earth's $\mathrm{N}_{2}$ atmosphere experienced less efficient thermal and nonthermal escape $\approx 3.0-4.0 \mathrm{Gyr}$ ago combined with plate tectonic related outgassing of primordial nitrogen, a much lower ${ }^{15} \mathrm{~N}$ isotope enrichment compared to Mars' atmosphere manifested, while the build up of its $\mathrm{N}_{2}$ atmosphere during the Archean. This suggests that the young Sun's early X-ray and EUV activity was lower as expected for a moderate or fast rotating young G-typ star.

In the case of Mars, gravity was too weak so that no nebular-based $\mathrm{H}_{2}$ envelope could accumulate during the planet's accretion phase. After Mars finalized its accretion after a few 
Myr and the planet's final magma ocean solidified, a steam atmosphere similar as on early Venus and Earth was outgassed, but with a lower amount of volatiles. From geological and mineralogical evidence early Mars was water-rich and depending on additional greenhouse gases than $\mathrm{CO}_{2}$, and impacts, liquid water most likely was present episodically on the planet's surface during the Noachian. Nitrogen, either originating from the magma ocean related outgassing, delivery of impactors, or from volcanic degassing, escaped to space, which is manifested in the strong enrichment of ${ }^{15} \mathrm{~N}$ isotopes against the lighter ${ }^{14} \mathrm{~N}$ isotopes in the present atmosphere. The efficient thermal escape decreased to negligible values at $\approx 3.8-4.0$ Gyr ago at the same time when the early magnetic dynamo stopped. At the same time period when the solar EUV flux decreased, volcanic activity most likely resulted in the build-up of a thin $\mathrm{CO}_{2}$ atmosphere during the late heavy bombardment phase $3.8-4.1 \mathrm{Gyr}$ ago, with a surface partial pressure that was $<0.5$ bar. This secondary $\mathrm{CO}_{2}$ atmosphere has then been lost by various nonthermal atmospheric escape processes (i.e., suprathermal atom escape, sputtering, ion pick up, cool ion escape) as well as surface weathering processes such as serpentinization.

For a better understanding of the origin and evolution of the atmospheres of early Venus, Earth and Mars it will be crucial to gain a clearer knowledge of the early evolution of the Sun, in particular of its EUV flux during the first Gyr. It is important to note that the terrestrial planets show significant "fingerprints" of the early history of the Solar System. By now, elemental and isotopical fractionations in the atmosphere and in the bulk composition of the Earth and to a lesser degree of Mars and Venus are known. Exactly these anomalies compared to their initial elemental abundances can give very important insights into the evolution of the planets related to the activity level of the young Sun. However, even though ranges of fractionations are also known for Mars and for Venus, it will be crucial to determine these ratios with more precision. All three planets evolved under the same solar activity at different orbital locations and their evolution has to be successfully described within the same model of the Sun's history. Future missions and landers like Venera-D to Venus, measuring isotope and element ratios like $\mathrm{K} / \mathrm{U}$ in more detail, will therefore play a key role within planetary sciences in the upcoming years and decades. A better knowledge will then also enhance our understanding of exoplanetary systems, in particular in view of the potential habitability of Earth-type exoplanets.

Acknowledgements H. Lammer, M. Güdel and M. Scherf acknowledge support by the Austrian Science Fund (FWF) NFN project S11601-N16, "Pathways to Habitability: From Disks to Active Stars, Planets and Life" and the related FWF NFN subprojects, S11604-N16 "Radiation \& Wind Evolution from the T Tauri Phase to ZAMS \& Beyond", S11606-N16 "Magnetospheres", S11607-N16 "Particle/Radiative Interactions with Upper Atmospheres of Planetary Bodies under Extreme Stellar Conditions". L. Noack acknowledges the collaboration with the COST Action TD 1308. S. Gebauer acknowledges support by the DFG project GZ: GR 2004/2-1 of the SPP 1833 "Building a Habitable Earth". M. Godolt acknowledges financial support from the German Research Foundation (DFG) Project GO 2610/1-1. L. Grenfell, M. Güdel and L. Noack acknowledge the collaboration within the COST Action TD 1308. H. Lammer also acknowledge stimulating discussions with B. Marty from the CRPG-CNRS, University of Nancy regarding isotope data analysis of carbonaceous chondrites and planetary atmospheres, E. Marcq from LATMOS/IPSL, UVSQ, Université Paris-Saclay on magma ocean related steam atmospheres, and L. Fossati from the Space Research Institute (IWF) of the Austrian Academy of Sciences (ÖAW). Furthermore, H. Lammer thanks L. Sproß from the University of Graz for the illustration shown in Fig. 16. The authors also thank the International Space Science Institute (ISSI) in Bern, the ISSI- 
Beijing team "Astrobiology" and the ISSI team"The Early Evolution of the Atmospheres of Earth, Venus, and Mars", and P. Odert for discussions on the losses of volatiles from planetary embryos and protoplanets. Finally the authors thank J. F. Kasting from the Department of Geosciences at the Penn State University and an anonymous referee for their suggestions and recommendations which helped to improve this work.

\section{References}

Abe Y, Matsui T (1985) The formation of an impact-generated $\mathrm{H}_{2} \mathrm{O}$ atmosphere and its implications for the early thermal history of the Earth. J Geophys Res 90:C545-C559

Abe Y (1997) Thermal and chemical evolution of the terrestrial magma ocean. Phys Earth Planet Interiors 100:27-39

Airapetian V S, Glocer A, Gronoff G, Hébrard E, Danchi W (2016) Prebiotic chemistry and atmospheric warming of early Earth by an active young Sun. Nature Geoscience 9:452-455

Albaréde F, Blichert-Toft J (2007) The split fate of the early Earth, Mars, Venus and Moon. Geochemistry 339:917-927

Albaréde F (2009) Volatile accretion history of the terrestrial planets and dynamic implications. Nature 461:1227-1233

Alexander C M O'D, Bowden R, Fogel M L, Howard K T, Herd C D K, Nittler N R (2012) The provenances of asteroids, and their contributions to the volatile inventories of the terrestrial planets. Science 337:721-723

Amerstorfer U V, Gröller H, Lammer H, Tian F, Noack L, Sherf M, Johnstone C, Tu L, Güdel M (2017) Escape and evolution of Mars' $\mathrm{CO}_{2}$ atmosphere: Influence of suprathermal atom. J Geophys Res, accepted

Anders E (1989) Pre-biotic organic matter from comets and asteroids. Nature 342:255Ú2 257

Atreya S K, Trainer M G, Franz H B, Wong M H, Manning H L K, Malespin C A, Mahaffy P R, Conrad P G, Brunner A E, Leshin L A, Jones J H, Webster C R, Owen T C, Pepin R O, Navarro-González R (2013) Primordial argon isotope fractionation in the atmosphere of Mars measured by the SAM instrument on Curiosity and implications for atmospheric loss. Geophys Res Lett 40:5605-5609

Aulbach S, Stagno V (2013) Evidence for a reducing Archean ambient mantle and its effects on the carbon cycle. Geology 44:751-754

Auvergne M, Bodin P, Boisnard L, Buey J-T, Chaintreuil S, and the CoRoT Team (2009) The CoRoT satellite in flight: description and performance. Astron Astrophys 506:411-424

Ayres T R (1997) Evolution of the solar ionizing flux. J. Geophys. Res. 102:1641Ú1651

Baes M, Gerya T, Sobolev S V (2016) 3D thermo-mechanical modeling of plume-induced subduction initiation. Earth Planet Sci Lett 453:193-203

Bakos G, Noyes R W, Kovács G, Stanek K Z, Sasselov D D, Domsa I (2004) Wide-field millimagnitude photometry with the HAT: A tool for extrasolar planet detection. Pub Astron Soc Pacific 116:266-277

Ballmer M D, Lourenago D L, Hirose K, Caracas R, Nomura R (2017) Reconciling magma-ocean crystallization models with the present-day structure of the earthŠs mantle. Geochemi Geophys Geosys 18:2785Ü2806 2017

Becker R H, Clayton R N, Galimov E M, Lammer H, Marty B, Pepin R O, Wieler R (2003) Isotopic signatures of volatiles in terrestrial planets. Space Sci Rev 106:377-410

Belousova E A, Kostitsyn Y A, Griffin W L, Begg G C, O’Reilly S Y, Pearson N J (2010) The growth of the continental crust: Constraints from zircon Hf-isotope data. Lithos 119:457Ú466

Berner R A, Kothvala Z (2001) GEOCARB III: A revised model of atmospheric $\mathrm{CO}_{2}$ over Phanerozoic time. Am J Sci 301:182-204

Bockelée-Morvan D, Crovisier J, Mumma M J, Weaver H A (2004) The composition of cometary volatiles. In: Festou M C, Keller H U, Weaver H A (eds) Comets 2, Univ Arizona Press, pp 391Ú423

Bodenheimer P, Pollack J B (1986) Calculations of the accretion and evolution of giant planets The effects of solid cores. Icarus 67:391-408

Bonsor A, Leinhardt Z M, Carter P J, Elliott T, Walter M J, Stewart S T (2014) A collisional origin to Earth's non-chondritic composition?. Icarus 257:291-300

Boyd E S, Peters J W (2013) New insights into the evolutionary history of biological nitrogen fixation. Front Microbiol 4:1-12

Borucki W J, Koch D, Basri,Gibor, Batalha N, Brown T, and the Kepler Team (2010) Kepler Planet-Detection Mission: Introduction and First Results. Science 327:977-980

Bottke W F, Vokrouhlický D, Minton D, Nesvorný D, Morbidelli A, Brasser R, Simonson B, Levison H F (2012) An Archaean heavy bombardment from a destabilized extension of the asteroid belt. Nature 485:78-81 
Bouhifd M A, Jephcoat A P (2011) Convergence of Ni and Co metal-silicate partion coefficients in the deep magma-ocean and coupled silicon-oxygen solubility in iron melts at high pressures. Earth Planet Sci Lett 307:341-348

Boyd S R, Philippot P (1998) Precambrian ammonium biochemistry: A study of the Moine metasediments, Scotland. Chem Geol 144:257-268

Bouvier A, Boyet M (2016) Primitive Solar System materials and Earth share a common initial ${ }^{142} \mathrm{Nd}$ abundance. Nature 537:399-402

Boyet M, Carlson R W (2005) ${ }^{142} \mathrm{Nd}$ evidence for early (>4.53 Ga) global differentiation of the silicate Earth. Science 309:576-581

Bösswetter A, Lammer H, Kulikov Yu N, Motschmann U, Simon S (2010) nonthermal water loss of the early Mars: 3D multi-ion hybrid simulations. Planet Space Sci 58:2013-2043

Brack A, Horneck G, Cockell C S, Bérces A, Belisheva N K, Eiroa C, Henning T, Herbst Tom, Kaltenegger L, Léger Alain, Liseau R, Lammer H, Selsis F, Beichman C, Danchi W, Fridlund M, Lunine J, Paresce F, Penny A, Quirrenbach A, Röttgering H, Schneider J, Stam D, Tinetti G, White G J (2010) Origin and evolution of life on terrestrial planets. Astrobiology 10:69-76

Brasser R (2013) The formation of Mars: building blocks and accretion time scale. Space Sc Rev 174:1-4

Brasser R, Matsumura S, Ida S, Mojzsis S J, Werner S C (2016a), Analysis of Terrestrial Planet Formation by the Grand Tack Model: System Architecture and Tack Location. Astrophys J 821:18-36

Brasser R, Mojzsis S J, Werner S C Matsumura S, Ida S (2016a) Late veneer and late accretion to the terrestrial planets. Earth Planet Sci Lett 455:85-93

Brasser R, Mojzsis S J, Matsumura S, Ida S (2017) The cool and distant formation of Mars. Earth Planet Sci Lett 468:85-93

Broeg C H, Benz W (2012) Giant planet formation: episodic impacts versus gradual core growth. Astron Astrophys 538:90, 13 pp

Brown M (2006) Duality of thermal regimes is the distinctive characteristic of plate tectonics since the Neoarchean. Geology 34:961-64

Buhler P B, Fasset C I, Head III J W, Lamb M P (2017) Timescales of fluvial activity and intermittency in Milna crater, Mars. Icarus 241:130-147

Buick R (2007) Did the Proterozoic ‘Canfield Ocean' cause a laughing gas greenhouse? Geobiology 5:97-100

Busigny V, Cartigny P, Philippot P (2011) Nitrogen isotopes in ophiolitic metagabbros: A re-evaluation of modern nitrogen fluxes in subduction zones and implication for the early Earth atmosphere. Geochim Cosmochim Acta 75:7502Ú7521

Busigny V, Bebout G E (2013) Nitrogen in the silicate Earth: Speciation and isotopic behavior during mineralfluid interactions. Elements 9:353-358

Caffee M W, Hudson G B, Velsko C, Alexander E C Jr, Huss G R, Chivas A R (1988) Non-atmospheric noble gases from $\mathrm{CO}_{2}$ well gases. In: Lunar Planet Sci XIX. Lunar Planetary Institute, Houston, pp 154-155

Caffee M W, Hudson G B, Velsko C, Huss G R, Alexander E C Jr, Chivas A R (1999) Primordial noble gases from EarthŠs mantle: Identification of a primitive volatile component. Science 285:2115-2118

Campbell I H, O’Neill H St C (2012) Evidence against a chondritic Earth. Nature 483:553-558

Catling D C, Kasting J F (2017) Atmospheric Evolution and Lifeless Worlds. Cambridge University Press

Cameron A G W (1983) Origin of the atmospheres of the terrestrial planets. Icarus 56:195-201

Canfield D E, Glazer A N, Falkowiski P G (2010) The evolution and future of Earth's nitrogen cycle. Science 330:192-196

Canil D (2002) Vanadium in peridotites, mantle redox and tectonic environments: Archean to present. Earth Planet Sci Lett 195:75-90

Canup R M (2004) Origin of terrestrial planets and the Earth-Moon system. Physics Today 57:56-62

Carlson C A, Ingraham J L (1983) Comparison of denitrification by Pseudomonas stutzeri, Pseudomonas aeruginosa, and Paracoccus denitrificans. Appl Environ Microbiol 45:1247-1253

Carr M H (1989) Recharge of the early atmosphere of Mars by impact-induced release of $\mathrm{CO}_{2}$. Icarus 79:311-327

Carr M H (1999) Mars. In: Beatty J K, Peterson C C, Chaikin A (eds) The New Solar System. Sky Publishing Corporation, Cambridge University Press, p 141

Carter P J, Leinhardt Z M, Elliott T, Walter M J, Stewart S T (2015) Compositional evolution during rocky protoplanet accretion. Astrophys J 813:72pp

Cartigny P, Marty B (2013) Nitrogen isotopes and mantle geodynamics: The emergence of life and the atmosphere-crust-mantle connection. Elements 9:359-366

Cassata W S (2017) Meteorite constraints on Martian atmospheric loss and paleoclimate. Earth Planet Sci Lett 479:322-329

Castillo-Rogez J, Johnson T V, Lee M H, Turner N J, Matson D L, Lunine J (2009) 26 Al decay: Heat production and a revised age for iapetus. Icarus 204:658Ú662 
Catling D C, Zahnle K J, McKay C P (2001) Biogenic methane, hydrogen escape, and the irreversible oxidation of early Earth. Science 293:839-844

Catling D C, Claire M W (2005) How Earth's atmosphere evolved to an oxic state: A status report. Earth Planet Sci Lett 237:1-20

Catling D C, Glein C, Zahnle K J, McKay C P (2005) Why $\mathrm{O}_{2}$ is required by complex life on habitable planets and the concept of planetary "oxygenation time". Astrobiology 5:415-438

Cawood P A, Kröner A, Pisarevsky S (2006) Precambrian plate tectonics: criteria and evidence. Geological Society of America Today 16:4-11

Chambers J.E., Wetherill G.W. (1998) Making the Terrestrial Planets: N-Body Integrations of Planetary Embryos in Three Dimensions, Icarus, 136, p. 304-327

Chambers J E (2001) Making more terrestrial planets. Icarus 152:205-224

Charnay B, Forget F, Wordsworth R, Leconde J, Millour E, Codron F, Spiga A (2013) Exploring the faint young Sun problem and the possible climates of the Archean Earth with a 3-D GCM. J Geophys Res 118:10414-10431

Charnay B, Hir G Le, Fluteau F, Catling D C (2017) A warm or a cold early Earth? New insights from a 3-D climate-carbon model. Earth and Planetary Sci Lett, 474:97-109

Chassefiére E (1996a) Hydrodynamic escape of hydrogen from a hot water-rich atmosphere: the case of Venus. J Geophys Res 101:26039-26056

Chassefiére E (1996b) Hydrodynamic escape of oxygen from primitive atmospheres: application to the cases of Venus and Mars. Icarus 124:537-552

Chassefiére E, Leblanc F (2011) Constraining methane release due to serpentinization by the observed D/H ratio on Mars. Earth Planet Sci Lett 310:262-271

Claire M W, Sheets J, Cohen M, Ribas I, Meadows V S, Catling D C (2012) The evolution of solar flux from $0.1 \mathrm{~nm}$ to $160 \mu \mathrm{m}$ : Quantitative estimates for planetary studies. Astrophys J 757, 95, $12 \mathrm{pp}$.

Clarke W B, Beg M A, Craig H (1969) Excess ${ }^{3} \mathrm{He}$ in the sea: evidence for terrestrial primordial helium. Earth Planet Sci Lett 6:213-220

Cloud P E Jr (1968) Atmospheric and hydrospheric evolution on the primitive Earth. Science 160:729-736

Cnossen I, Sanz-Forcada J, Favata F, Witasse O, Zegers T, Arnold N F (2007) Habitat of early life: Solar X-ray and UV radiation at Earth's surface 4-3.5 billion years ago. J Geophys Res 112:E2

Conrad P G, Malespin C A, Franz H B, Pepin R O, Trainer M G, Schwenzer S P, Atreya S K, Freissinet C, Jones J H, Manning H, Owen T, Pavlova A A, Wiens R C, Wong M H, Mahaffy P R (2016) In situ measurement of atmospheric krypton and xenon on Mars with Mars Science Laboratory. Earth Planet Sci Lett 454:1-9

Craig H, Lupton J E (1976) Primordial neon, helium, and hydrogen in oceanic basalts. Earth Planet Sci Lett 31:369-385

Cubillos P, Erkaev E V, Juvan I, Fossati L, Johnstone C P, Lammer H, Lendl M, Odert P, Kislyakova K G (2017) An overabundance of low-density Neptune-like planets. MNRAS 466:1868-1879

Dalsgaard T, Thamdrup B, Canfield D E (2005) Anaerobic ammonium oxidation (anammox) in the marine environment. Limnol Oceanogr 54:1643-1652

Dauphas N, Kasting J F (2011) Low $\mathrm{PCO}_{2}$ in the pore water not in the Archean air. Nature 474:E2

Dauphas N, Pourmand A (2011) Hf-W-Th evidence for rapid growth of Mars and its status as a planetary embryo. Nature 473:489-492

Debaille V, Brandon A D, Yin Q Z, Jacobsen B (2007) Coupled ${ }^{142} \mathrm{Nd}-{ }^{143} \mathrm{Nd}$ evidence for a protracted magma ocean in Mars. Nature 450:525-528

Dehant V, Lammer H, Kulikov Yu N, Grießmeier J-M, Breuer D, Verhoeven O, Karatekin Ö van Hoolst T, Korablev O, Lognonné P (2007) Planetary magnetic dynamo effect on atmospheric protection of early Earth and Mars. Space Sci Rev 129:279-300

Delano J W (2001) Redox History of the Earth's Interior since 3900 Ma: Implications for Prebiotic Molecules. Orig Life Evol Biosph 31:311-341

Dhuime B, Hawkesworth C J, Cawood P A, Storey C D (2012) A change in the geodynamics of continental growth 3 billion years ago. Science 335:1334-1336

Dhuime B, Wuestefeld A, Hawkesworth C J (2015) Emergence of modern continental crust about 3 billion years ago. Nature Geoscience 8:552-555

Dixon, E T, Honda M, McDougall I, Campbell I H, Sigridsson I (2000) Preservation of neon-solar neon isotopic ratios in Icelandic basalds. Earth Planet Lett 180:309-324

Drake M J, Righter K, (2002) Determining the composition of the Earth. Nature 416:39-44

Dohm J M, Baker V R, Boynton W V, Fairén A G, Ferris J C, Finch M, Furfaro R, Hare T M, Janes D M, Kargel J S, Karunatillake S, Keller J, Kerry K, Kimi K J, Komatsu G, Mahaneyk W C, Schulze-Makuch D, Marinangeli L, Ori G G, Ruiz J, Wheelock S J (2005) GRS evidence and the possibility of paleooceans on Mars. Planet Space Sci 57:664-684 
Dodd M S, Papineau D, Grenne T, Slack J F, Rittner M, Pirajno F, O’Neil J, Little C T S (2017) Evidence for early life in Earth's oldest hydrothermal vent precipitates. Nature 543:60-65

Domagal-Goldman S D, Segura A, Claire M W, Robinson T D, Meadows V S (2014) Abiotic ozone and oxygen in atmospheres similar to prebiotic Earth. Astrophys J 792, 15, p 90

Driese S G, Jirsa M A, Minghua Ren, Brantley S L, Sheldon N D, Parker D, Scmitz M (2011) Neoarchean paleoweathering of tonalite and metabasalt: Implications for reconstructions of $2.69 \mathrm{Ga}$ early terrestrial ecosystems and paleoatmospheric chemistry. Precambrian Research 189:1-17

Elkins-Tanton L T (2008) Linked magma ocean solidifcation and atmospheric growth for Earth and Mars. Earth Planet Sci Lett 271:181-191

Elkins-Tanton L T (2011) Formation of early water oceans on rocky planets. Astrophys Space Sci 332:359-364

Elkins-Tanton L T (2012) Magma oceans in the inner solar system. Annu Rev Earth Planet Sci 40:113-139

Erkaev N V, Lammer H, Odert P, Kulikov Yu N, Kislyakova K G, Khodachenko M L, Güdel M, Hanslmeier A, Biernat H K (2013) XUV exposed non-hydrostatic hydrogen-rich upper atmospheres of terrestrial planets. Part I: Atmospheric expansion and thermal escape. Astrobiology 13:1011-1029

Erkaev N V, Lammer H, Elkins-Tanton L T, Stökl A, Odert P, Marcq E, Dorfi E A, Kislyakova K G, Kulikov Yu N, Leitzinger M, Güdel M (2014) Escape of the martian protoatmosphere and initial water inventory. Planet Space Sci 98:106-119

Fairén A G (2017) Icy Mars lakes warmed by methane. Nature Geoscience 10:717-718

Farquhar J, Zerkle A L, Bekker A (2014) Geologic and geochemical constraints on Earth's early atmosphere, In: Holland D H, Turekian K (eds) Treatise in Geochemistry: Reference module in Earth systems and environmental sciences. 6:91-138

Fassett C I, Head J W III (2005) New evidence for fluvial sedimentary deposits on Mars: Deltas formed in a crater lake in the Nili Fossae region. Geophys Res Lett 32:L14201

Feigelson E D, Garmire G P, Pravdo S H (2002) Magnetic flaring in the pre-main-sequence Sun and implications for the early solar system. Astrophys J 572:335-349

Fennel K, Follows M, Falkowski P (2005) The co-evolution of the nitrogen, carbon and oxygen cycles in the Proterozoic Ocean. American Journal of Science 305: 526-45

Feulner, G (2012) The faint young Sun problem. Rev Geophys 50:1-29

Fischer T P, Hilton D R, Zimmer M M, Shaw A M, Sharp Z D, Walker J A (2002) Subduction and recycling of nitrogen along the Central American margin. Science 297:1154-1157

Fischer-Gödde M, Kleine T (2017) Ruthenium isotopic evidence for an inner solar system origin of the later vaneer. Nature 541:525-527

Flament N, Coltice N, Rey P F (2008) A case for late-Archaean continental emergence from thermal evolution models and hypsometry. Earth Planet Sci Lett 275:326-336

Forget F, Wordsworth R, Millour E, Madeleine J-B, Kerber L, Leconte J, Marcq E, Haberle R M (2013) $3 \mathrm{D}$ modelling of the early Martian climate under a denser $\mathrm{CO}_{2}$ atmosphere: temperatures and $\mathrm{CO}_{2}$ ice clouds. Icarus 222:81Û́99

Fossati, L, Erkaev N V, Lammer H, Cubillos P E, Odert P, Juvan I, Kislyakova K G, Lendl M, Kubyshkina D, Bauer S J (2017) Aeronomical constraints to the minimum mass and maximum radius of hot low-mass planets. Astron Astrophys 598:A90, 9 pp

Fowler D, Coyle M, Skiba U, Sutton M A, Cape J N, Reis S, Sheppard L J, Jenkins A, Grizzetti B, Galloway J N, Vitousek P, Leach A, Bowman A F, Butterbach-Bahl K, Dentener F, Stevenson D, Amann M, Voss M (2013) The global nitrogen cycle in the twenty-first century. Phil Trans Royal Soc B 368, 1621, DOI: $10.1098 /$ rstb.2013.0164

Fox J L (1993) The production and escape of nitrogen atoms on Mars. J Geophys Res 98:3297-3310

Frey H V (2006) Impact constraints on the age and origin of the lowlands of Mars. GRL 33, L08S02

Fridlund M, Eiroa C, Henning T, Herbst T, Kaltenegger L, Léger A, Liseau R, Lammer H, Selsis F, Beichman C, Danchi W, Lunine J, Paresce F, Penny A, Quirrenbach A, Röttgering H, Schneider J, Stam D, Tinetti G, White G J (2010) A roadmap for the detection and characterization of other Earths. Astrobiology 10:113-119

Frost D J, Liebske C, McCammon C, Rubie D C (2008) The redox state of Earth's mantle. Annu Rev Earth Planet Sci 36:389-420

Füri E, Marty B (2015) Nitrogen isotope variations in the solar system. Nature Geoscience 8:515-522

Gaillard F, Scaillet B, Arndt N T (2011) Atmospehric oxygenation caused by a change in volcanic degassing pressure. Nature 478:229-232

Garvin J, Buick R, Anbar A D, Arnold G L, Kaufman A J (2011) Isotopic evidence for an aerobic nitrogen cycle in the latest Archean. Science 323: 1045-48

Gallet F, Bouvier J (2013) Improved angular momentum evolution model for solar-like stars. Astron Astrophys 556, A36, $15 \mathrm{pp}$ 
Galloway J N (2003) The global nitrogen cycle. In: Holland H D, Turekian K K (eds) Treatise on Geochemistry. Pergamon, Oxford, pp 557-583

Gebauer S, Grenfell J L, Stock J W, Lehmann R, Godolt M, von Paris P, Rauer H (2017) Evolution of Earthlike extrasolar planetary atmospheres: Assessing the atmospheres and biospheres of early Earth analog planets with a coupled atmosphere biogeochemical model. Astrobiology 17:27-54

Geiss J (1973) Solar wind composition and implications about the history of the solar system. Internat Cosmic Ray Conf, 13th Conf Papers, Univ Denver, 5:3375-3398

Genda H, Abe Y (2003) Survival of a proto-atmosphere through the stage of giant impacts: the mechanical aspects. Icarus 164:149-162

Genda H, Abe Y (2005) Enhanced atmospheric loss on protoplanets at the giant impact phase in the presence of oceans. Nature 433:842-844

Gerya T V, Stern R J, Baes M, Sobolev S V, Whattam S A (2015) Plate tectonics on the Earth triggered by plume-induced subduction initiation. Nature 527:221-225

Gillmann C, Chassefiére E, Lognonné P (2009) A consistent picture of early hydrodynamic escape of Venus atmosphere explaining present $\mathrm{Ne}$ and $\mathrm{Ar}$ isotopic ratios and low oxygen atmospheric content. Earth Planet Sci Lett 286:503-513

Godfrey L V, Falkowski P G (2009) The cycling and redox state of nitrogen in the Archaean ocean. Nature Geosci 2:725-729

Goldblatt C (2008) Bistability of atmospheric oxygen, the Great Oxidation and climate. PhD thesis

Goldblatt C, Claire M W, Lenton T M, Matthews A J, Watson A J, Zahnle K J (2009) Nitrogen-enhanced greenhouse warming on early Earth. Nature Geoscience 2:891-896

Goldblatt C, Zahnle K J (2011) Faint Young Sun Paradox remains. Nature 474:E1

Greber N D, Dauphas N, Bekker A, Ptáček M P (2017) Titanium isotopic evidence for felsic crust and plate tectonics 3.5 billion years ago. Science 357:1271-1274

Grott M, Morschhauser A, Breuer D, Hauber E (2011) Volcanic outgassing of $\mathrm{CO}_{2}$ and $\mathrm{H}_{2} \mathrm{O}$ on Mars. Earth Planet Sci Lett 308:391-400

Grenfell J L, Gebauer S, von Paris P, Godolt M, Hedelt P, Patzer A B C, Stracke B, Rauer H (2011) Sensitivity of biomarkers to changes in chemical emissions in the EarthŠs Proterozoic atmosphere. Icarus 211:81-88

Gruber N (2008) The marine nitrogen cycle: Overview and challenges. In Nitrogen in the Marine Environment (eds Capnoe D G, Bronk D A, Mulholland M R, Carpenter E J) Elsevier, pp 1-50

Güdel M, Guinan E F, Skinner S L (1997) The X-ray Sun in time: A study of the long-term evolution of coronae of solar-type stars. Astrophys J 483:947-960

Güdel M (2007) The Sun in time: Activity and environment. Living Rev Solar Phys 4:3, 137 pp

Haendel D, Mühle K, Nitzsche H-M, Stiehl G, Wand U (1986) Isotopic variations of the fixed nitrogen in metamorphic rocks. Geochim Cosmochim Acta 50:749Ü758

Halama, R., Bebout, G. E., John, T., \& Scambelluri, M. (2014). Nitrogen recycling in subducted mantle rocks and implications for the global nitrogen cycle. Int J Earth Sci 103:2081-2099

Halliday A N, Wänke H, Birck J L, Clayton R N (2001) The accretion, composition and early differentiation of Mars. Space Sci Rev 96:197-230

Halliday A N (2014) The origin and earliest history of the Earth. In: Davis A M (ed) Planets, asteroids, comets and the solar system. Tratise on Geochemistry, pp 149-211

Hamano K, Abe Y, Genda H (2013) Emergence of two types of terrestrial planet on solidification of magma ocean. Nature 497:607-610

Hamilton W B (2011) Plate tectonics began in Neoproterozoic time, and plumes from deep mantle have never operated. Lithos 123:1-20

Hansen B M S (2009) Formation of the terrestrial planets from a narrow annulus. Astrophys J 703:1131-1140

Harper C L, Jacobsen S B (1996) Noble gases and Earth's accretion. Science 273:1814-1818

Haqq-Misra J D, Domagal-Goldman S D, Kasting P J, Kasting J F (2008) A revised, hazy methane greenhouse for the Archean Earth. Astrobiology 8:1127-1137

Hedges S B (2002) The origin and evolution of model organisms. Nature 3:838-848

Hessler A M, Lowe D R, Jones R L, Bird D K (2004) A lower limit for atmospheric carbon dioxide levels 3.2 billion years ago. Nature 428:736-738

Hébrard E, Marty B (2014) Coupled noble gas-hydrocarbon evolution of the early Earth atmosphere upon solar UV irradiation. Earth Planet Sci Lett 385:40-48

Hier-Majumder S, Hirschmann M M (2017) The origin of volatiles in the Earth's mantle, Geochem. Geophys. Geosyst. 18, 3078-3092

Hillenbrand L A (2008) Disk-dispersal and planet-formation timescales. Physica Scripta, 130, id. 014024

Hin R C, Coath C D, Carter P J, Nimmo F, Lai Yi-Jen, Pogge von Strandmann P A E, Willbold M, Leinhardt Z M, Walter M J, Elliot T (2017) Magnesium isotope evidence that accretional vapour loss shapes planetary compositions. Nature 549:511-515 
Hirschmann M M (2009) Ironing out the oxidation of Earth's mantle. Science 325:545-546

Hirschmann M M (2010) Partial melt in the oceanic low velocity zone. Phys Earth Planet Interiors 179:60-71

Höning D, Hansen-Goos H, Airo A, Spohn T (2014) Biotic vs. abiotic Earth: A model for mantle hydration and continental coverage. Planet Space Sci 98, 5-13

Hoffmann J H, Oyama V I, Zahn U V (1980) Measurements of the lower atmospheric composition: A comparison of results. J Geophys Res 85:7871-7881

Holland H D (1962) Petrologic studies. In: Buddington A F, Engel E J, James H L, Leonard B F (eds) A volume to honor. Geological Society of America, New York, pp 447-477

Holland H D (1978) The chemistry of the atmosphere and oceans. Wiley, New York, p 351

Holland H D (1984) The chemical evolution of the atmosphere and oceans. Princeton Univ Press, Princeton, $598 \mathrm{pp}$

Holm N G (1992) Marine hydrothermal systems and the origins of life. Orig Life Evol Biosph 22:181-242

Honda M, McDougall I, Patterson D B, Doulgeris A, Clague D A (1991) Possible solar noble-gas component in Hawaiian basalts. Nature 349:149-151

Holloway J M, Dahlgren R A (2002) Nitrogen in rock: Occurences and biogeochemical implications. Global Biochem Cycles 16:1-17

Hopkins M, Harrison T M, Manning C E (2008) Low heat flow inferred from > 4 Gyr zircons suggests Hadean plate boundary interactions. Nature 456:493-96

Howe A R, Burrows A, Verne W (2014) Mass-radius relations and core-envelope decompositions of superEarths and sub-Neptunes. Astrophys J 787, 173A, 22 pp

Hutchins K S, Jakosky B M (1996) Evolution of Martian atmospheric argon: Implications for sources of volatiles. J Geophys Res 101:14933-14950

Iijima Y, Goto K, Minoura K, Komatsu G, Imamura F (2014) Hydrodynamics of impact-induced tsunami over the Martian ocean. Planet Space Sci 95:33-44

Ikoma M, Nakazawa K, Emori H (2000) Formation of giant planets: dependences on core accretion rate and grain opacity. Astrophys J 537:1013-1025

Ikoma M, Genda H (2006) Constraints on the mass of a habitable planet with water of nebular origin. Astrophys J 648:696-706

Ikoma M, Hori Y (2012) In situ accretion of hydrogen-rich atmospheres on short-period super-Earths: implications for the Kepler-11 planets. Astrophys J 753:66, 6pp

Jackson J (2002) Strength of the continental lithosphere: time to abandon the jelly sandwich? GSA today 12, 4-9

Jacob D J (1999) Introduction to atmospheric chemistry. Princeton University Press, pp 266

Jacobson S A, Morbidelli A, Raymond S N, O'Brien D P, Walsh K J, Rubie D C (2014) Highly siderophile elements in EarthŠs mantle as a clock for the Moon-forming impact. Nature 508:84-87

Jacobsen S B (2005) The Hf-W isotopic system and the origin of the Earth and Moon. Annu Rev Earth Planet Sci 33:531-570

Jakosky B M, Pepin R O, Johnson R E, Fox J L (1994) Mars atmospheric loss and isotopic fractionation by solar-wind-induced sputtering and photochemical escape. Icarus 111:271-288

Jakosky B M, Slipski M, Benna M, Mahaffy P, Elrod M, Yelle R, Stone S, Alseed N (2017) Science 355:14081410

Jellinek A M, Jackson M G (1995) Conenctions between the bulk composition, geodynamics and habitability of Earth. Nature Geoscience 8:587-593

Johansen A, Blum J, Tanaka H, Ormel C, Bizzarro M, Rickman H (2014) The multifaceted planetesimal formation process. In: Beuther H, Kessen R S, Dullemond C P, Henning T (eds) Protostars and Planets VI Univ. Arizona Press, pp 547-570

Johnson B, Goldblatt C (2015) The nitrogen budget of Earth. Earth Sci Rev 148:150-173

Johnstone C P, Güdel M, Stökl A, Lammer H, Tu L, Kislyakova K G, Lüftinger T, Odert P, Erkaev N V, Dorfi E A (2015a) The evolution of stellar rotation and the hydrogen atmospheres of habitable-zone terrestrial planets. Astrophys J Lett 815, id. A12, 6 pp

Johnstone C P, Güdel M, Lüftinger T, Toth G, Brott I (2015b) Stellar winds on the main-sequence. I. Wind model. Astron Astrophys 577:27, 22pp

Johnstone C P, Güdel M, Brott I, Lüftinger T (2015c) Stellar winds on the main-sequence. II. The evolution of rotation and winds. Astron Astrophys 577:28, $25 \mathrm{pp}$

Kanzaki Y, Murakami T (2015) Estimates of atmospheric $\mathrm{CO}_{2}$ in the Neoarchean-Paleoproterocoic from paleosols. Geochimica et Cosmochimica Acta 159:190-219

Karki B B, Stixrude L P (2010) Viscosity of $\mathrm{MgSiO}_{3}$ liquid at EarthS̆s mantle conditions: implications for an early magma ocean. Science 328:740Ü742

Karato S-I (2011) Water distribution across the mantle transition zone and its implications for global material circulation. Earth and Planetary Science Letters 301, 413-423 
Kasting J F, Donahue T M (1980) The evolution of atmospheric ozone. J Geophys Res 85:3255-3263

Kasting J F (1982) Stability of ammonia in the primitive terrestrial atmosphere. J Geophys Res 87:3091-3098

Kasting J F, Pollack J B (1983) Loss of water from Venus. I. Hydrodynamic escape of hydrogen. Icarus 53:479-508

Kasting J F, Pollack J B, Crisp D (1984) Effects of high $\mathrm{CO}_{2}$ levels on surface temperature and atmospheric oxidation state of the early earth. J Atm Chem 1:403-428

Kasting J F, Pollack J B, Ackerman T P (1984) Response of Earth's atmosphere to increases in solar flux and implications for loss of water from Venus. Icarus 57:335-355

Kasting J F (1988) Runaway and moist greenhouse atmospheres and the evolution of Earth and Venus. Icarus 74:472-494

Kasting J F (1992) Paradox lost and paradox found. Nature 355:676-77

Kasting J F, Brown L L, Acord J M, Pollack J B (1992) Was early Mars warmed by ammonia? In: Workshop on the Martian surface and atmosphere through time. Lunar and Planetary Inst, 84-85

Kasting J F (1993) Earth's early atmosphere. Nature 259:920-926

Kasting J F, Whitmire D P, Reynolds R T (1993a) Habitable zones around main sequence stars. Icarus 101:108-128

Kasting J F, Eggler D H, Raeburn S P (1993b) Mantle redox evolution and the case for a reduced Archean atmosphere. J Geology 101:245-257

Kasting J F, Schultz P A (1996) Reservoir time-scales for anthropogenic $\mathrm{CO}_{2}$ in the atmosphere: commentary. Tellus B 48:5, p 703

Kasting J F (1998) Habitable zones around stars and the search for extraterrestrial life. American Astron Soc, 193rd AAS Meeting, Bull American Astron Soc 30, p 1328

Kasting J F, Ono S (2006) Palaeoclimates: the first two billion years. Philos Trans R Soc Lond B Biol Sci 361:917-29

Kasting J F, Siefert J L (2011) Biogeochemistry. The nitrogen fix. Nature 412:26-27

Kasting J F (2013) What caused the rise of atmospheric O2. Chemical Geology 362:13-25

Kelly K A, Cottrell E (2009) Water and the oxidation state of subduction zone magmas. Science 325:605-607

Kelley K A, Plank T, Newman S, Stolper E M, Grove T L, Parman S, Hauri, E H (2010) Mantle melting as a function of water beneath the Mariana arc. J Petrol 51:1711-1738

Kerber L, Forget F, Wordsworth R (2015) Sulfur in the early Martian atmosphere revisited: Experiments with a 3-D Global Climate Model. Icarus 261:133Ü148

Kharecha P, Kasting J F, Siefert J (2005) A coupled atmosphere Ú ecosystem model of the early Archean Earth. Geobiology 3:53-76

Kiehl J T, Dickinson R E (1987) A study of the radiative effects of enhanced atmospheric $\mathrm{CO}_{2}$ and $\mathrm{CH}_{4}$ on early Earth surface temperatures. J Geophys Res 92:2991-2998

Kite E S, Williams J-P, Lucas A, Aharonson O (2014) Low palaeopressure of the martian atmosphere estimated from the size distribution of ancient craters. Nature Geoscience 7:335-339

Kleine T, Münker C, Mezger K, Palme H (2002) Rapid accretion and early core formation on asteroids and the terrestrial planets from Hf-W chronometry. Nature 418:952-955

Kleine T (2011) Earth's patchy late veneer. Nature 477:168-169

Kraft R P (1967) Studies of stellar rotation. V. The dependence of rotation on age among solar-type stars. Astrophys 150:551

Kokubo E, Ida S (2000) Formation of protoplanets from planetesimals in the solar nebula. Icarus 143:15-27

Komiya T, Maruyama S, Masuda T, Nohda S, Hayashi M, Okamoto K (1999) Plate tectonics at 3.8-3.7 Ga: field evidence from the Isua accretionary complex, southern West Greenland. J. Geol. 107:515-54

Kopp R E, Kirschvink J L, Hilburn I A, Nash C Z (2005) The Paleoproterozoic snowball Earth: a climate disaster triggered by the evolution of oxygenic photosynthesis. PNAS 102:11131-11136

Korenaga J (2008) Plate tectonics, flood basalts, and the evolution of Earth's oceans. Terra Nova 20:419-39

Korenaga J (2013) Initiation and evolution of plate tectonics on Earth: theories and observations. Ann Rev Earth Planet Sci 41:117-151

Kuhn W R, Atreya S K (1979) Ammonia photolysis and the greenhouse effect in the primordial atmosphere of the Earth. Icarus 37:207-213

Kulikov Yu N, Lammer H, Lichtenegger H I M, Terada N, Ribas I, Kolb C, Langmayr D, Lundin R, Guinan E F, Barabash S, Biernat H K (2006) Atmospheric and water loss from early Venus. Planet Space Sci 54:1425-1444

Kulikov Yu N, Lammer H, Lichtenegger H I M, Penz T, Breuer D, Spohn T, Lundin R, Biernat H K (2007) A comparative study of the influence of the active young Sun on the early atmospheres of Earth, Venus, and Mars. Space Sci Rev 129:207-243

Kunze M, Godolt M, Langematz U, Grenfell J L, Hamann-Reinus A, Rauer H (2014) Investigating the early Earth faint young Sun problem with a general circulation model. Planet Space Sci 98:77-92 
Kurokawa H, Kurosawa K, Usui T (2017) A lower limit of atmospheric pressure on early Mars inferred from nitrogen and argon isotopic compositions. Icarus 299:443-459

Lammer H, Kasting J F, Chassefiére E, Johnson R E, Kulikov Yu N, Tian F (2008) Atmospheric escape and evolution of terrestrial planets and satellites. Space Sci Rev 139:399-436

Lammer H, Bredehöft J H, Coustenis A, Khodachenko M L, Kaltenegger L, Grasset O, Prieur D, Raulin F, Ehrenfreund P, Yamauchi M, Wahlund J-E, Grießmeier J-M, Stangl G, Cockell C S, Kulikov Yu N, Grenfell J L, Rauer H (2009) What makes a planet habitable? Astron Astrophys Rev 17:181-249

Lammer H, Kislyakova K G, Odert P, Leitzinger M, Schwarz R, Pilat-Lohinger E, Kulikov Yu N, Khodachenko M L, Güdel M, Hanslmeier A (2011) Pathways to Earth-like atmospheres extreme ultraviolet (EUV)powered escape of hydrogen-rich protoatmospheres. Orig Life Evol Biosphere 41:503-522

Lammer H, Chassefiére E, Karatekin Ö, Morschhauser A, Niles P B, Mousis O, Odert P, Möstl U V, Breuer D, Dehant V, Grott M, Gröller H, Hauber E, San Pham, Lê Binh (2013) Outgassing history and escape of the martian atmosphere and water inventory. Space Sci Rev 174:113-154

Lammer H, Stökl A, Erkaev N V, Dorfi E A, Odert P, Güdel M, Kulikov Yu N, Kislyakova K G, Leitzinger M (2014) Origin and loss of nebula-captured hydrogen envelopes from 'sup'- to 'super-Earths' in the habitable zone of Sun-like stars. MNRAS 439:3225-3238

Lammer H, Erkaev N V, Fossati L, Juvan I, Odert P, Cubillos P E, Guenther E, Kislyakova K G, Johnstone C P, Lüftinger T, Güdel M (2016) Identifying the 'true' radius of the hot sub-Neptune CoRoT-24b by mass-loss modelling. MNRAS 461:L62-L66

LaTourrette T, Wasserburg G J (1998) Mg diffusion in anorthite: implications for the formation of early solar system planetesimals. Earth Planet Sci Lett 158:91-108

Lebrun T, Massol H, Chassefiére E, Davaille A, Marcq E, Sarda P, Leblanc F, Brandeis G (2013) Thermal evolution of an early magma ocean in interaction with the atmosphere. J Geophys Res (Planets) 118:11551176

Levison H F, Morbidelli A, Tsiganis K, Nesvorny D, Gomes R (2011) Late orbital instabilities in the outer planets induced by interaction with a self-gravitating planetesimal disk. Astron J 142, $11 \mathrm{pp}$

Li Y, Wiedenbeck M, Shcheka S, Keppler H (2013) Nitrogen solubility in upper mantle minerals. Earth Planet Sci Lett 377:311-323

Li Y, Keppler H (2014) Nitrogen speciation in mantle and crustal fluids. Geochim Cosmochim Acta 129:13-32

Li Z-X A, Lee C-T A (2004) The constancy of upper mantle $\mathrm{fO}_{2}$ through time inferred from V/Sc ratios in basalts. Earth Planet Sci Lett 228:483-493

Lichtenegger H I M, Lammer H, Grießmeier J-M, Kulikov Yu N, von Paris P, Hausleitner W, Krauss S, Rauer H, Kulikov Yu N, von Paris P, Hausleitner W, Krauss S, Rauer H (2010) Aeronomical evidence for higher $\mathrm{CO}_{2}$ levels during Earth's Hadean epoch. Icarus 210:1-7

Lichtenegger H I M, Kislyakova K G, Odert P, Erkaev N V, Lammer H, Gröller H, Johnstone C P, ElkinsTanton L, Tu L, Güdel M, Holmström M (2016) Solar XUV and ENA-driven water loss from early Venus' steam atmosphere. J Geophys Res 121:4718-4732

Lillis R J, Frey H V, Manga M (2008) Rapid decrease in Martian crustal magnetization in the Noachian era: Implications for the dynamo and climate of early Mars. Geophys Res Lett 35:L14203

Lillis R J, Robbins S, Manga M, Halekas J S (2013) Time history of the Martian dynamo from crater magnetic field analysis. J Geophys Res 118:1-24

Lin D N C, Papaloizou J (1986) On the tidal interaction between protoplanets and the protoplanetary disk. III - Orbital migration of protoplanets, Astrophys J 309:846-857

Lu W, Cang X, Howard A D (2017) New Martian valley network volume estimate consistent with ancient ocean and warm and wet climate. Nature Communications 8:15766

Luger R, Barnes R, Lopez E, Fortney J, Jackson B, Meadows V (2015) Habitable evaporated cores: Transforming mini-Neptunes into super-Earths in the habitable zones of M dwarfs. Astrobiology 15:57-88

Lumair G W, Shukolyukov A (1998) Early solar system timescales according to ${ }^{53} \mathrm{Mn}-{ }^{53} \mathrm{Cr}$ systematics. Geochimica et Csmochimica Acta 62:2863-2886

Lunine J I, O'Brien S P, Raymond S N, Morbidelli A, Quinn T, Graps A L (2011) Dynamic models of terrestrial planet formation. Adv Sci Letters 4:325-338

Luo G, Ono S, Beukes NJ, Wang DT, Xie S, Summons RE. 2016. Rapid oxygenation of Earth's atmosphere 2.33 billion years ago. Science Adv 2:1-9

Lyons T W, Reinhard C T, Planavsky N J (2014) The rise of oxygen in Earth's early ocean and atmosphere. Nature 506:307-315

Maggio A, Sciortino S, Vaiana G S, Majer P, Bookbinder J A, Golub L, Harnden F R Jr, Rosner R (1987) Einstein Observatory survey of X-ray emission from solar-type stars - The late F and G dwarf stars. Astrophys 315:687-699

Maindl T I, Dvorak R, Lammer H, Güdel M, Schäfer C, Speith R, Odert P, Erkaev N V, Kislyakova K G, PilatLohinger E (2015) Impact induced surface heating by planetesimals on early Mars. Astron Astrophys 
574:A22, $7 \mathrm{pp}$

Mahaffy P R, Webster Ch R, Atreya S K, Franz H, Wong M, and the MSL Science Team (2013) Abundance and isotopic composition of gases in the Martian atmosphere from the Curiosity rover. Science 341:263-266

Maher K A, Stevenson D J (1988) Impact frustration of the origin of life. Nature 331:612-614

Mamyrin B A, Tolstikhin I N, Anufriev G S, Kamensky I L (1969) Anomalous isotopic composition of helium in volcanic gases. Dokl Akad Nauk SSSR 184:1197-1199

Manning C V, Zahnle K J, McKay C P (2009) Impact processes of nitrogen on early Mars. Icarus 199:273-285

Marcq E (2012) A simple 1-D radiative-convective atmospheric model designed for integration into coupled models of magma ocean planets. J Geophys Res (Planets) 117, E01001

Marty B, Allé (1994) Neon and Argon isotope constrains on Earth-atmosphere evolution (ed Matsuda J). Noble Gas Geochem Cosmochem pp. 191-204

Marty B (1995) Nitrogen content of the mantle inferred from $\mathrm{N}_{2}$-Ar correlation in oceanic basalts. Nature 377:326-329

Marty B (2012) The origins and concentrations of water, carbon, nitrogen and noble gases on Earth. Earth Planet Sci Lett 313:56-66

Marty B, Dauphas N (2003) The nitrogen record of crust-mantle interaction and mantle convection from Archean to present. Earth Planet Sci Lett 206:397-410

Marty B, Yokochi R (2006) Water in the Earth. Rev Mineral Geochem 62:421-450

Marty B, Meibom A (2007) Noble gas signature of the Late Heavy Bombardment in the Earth's atmosphere. eEarth 2:43-49

Marty B, Zimmermann L, Pujol M, Burgess R, Philippot P (2013) Nitrogen isotopic composition and density of the Archean atmosphere. Science 342:101-104

Maurice M, Tosi N, Samuel H, Plesa A-C, Hüttig C, Breuer D (2017) Onset of solid-state mantle convection and mixing during magma ocean solidification. J Geophys Res Planets 122:577Ú 598

Masset F, Snellgrove M (2001) Reversing type II migration: resonance trapping of a lighter giant protoplanet. MNRAS 320:L55-L59

Masset F S, Papaloizou J C B (2003) Runaway migration and the formation of hot Jupiters. Astrophys J 588:494-508

Massol H, Hamano K, Tian F, Ikoma M, Abe Y, Chassefiére E, Davaille A, Genda H, Güdel M, Hori Y, Leblanc F, Marcq E, Sarda P, Shematovich V I, Stökl A, Lammer H (2016) Formation and evolution of protoatmospheres. Space Sci Rev 205:153-211

Maurette M, Brack A (2006) Cometary petroleum in Hadean time? Meteorit Planet Sci 41:52-47

Meshik A, Hohenberg C Pravdivtseva O, Burnett D (2014) Heavy noble gases in solar wind delivered by Genesis mission. Geochim Cosmochim Acta 127:326-347

Mikhail S, Sverjensky D A (2014) Nitrogen speciation in upper mantle fluids and the origin of Earth's nitrogen-rich atmosphere. Nature Geoscienve 7:816-819

Miyakawa S, Cleaves H J, Miller S L (2002) The cold origin of life: B. implications based on pyrimidines and purines produced from frozen ammonium cyanide solutions. Orig Life Evol Biosph 32:209-218

Mizuno H, Nakazawa K, Hayashi C (1980) Dissolution of the primordial rare gases into the molten Earth's material. Earth Planet Sci Lett 50:202-210

Mizuno H, Wetherill G W (1984) Grain abundance in the primordial atmosphere of the Earth. Icarus 59:74-86

Monteux J, Andrault D, Samuel H (2016) On the cooling of a deep terrestrial magma ocean. Earth Planet Sci Lett 448:140Ú149

Mojzsis S J, Harrison T M, Pidgeon R T (2001) Oxygen-isotope evidence from ancient zircons for liquid water at the Earth's surface 4300 Myr ago. Nature 409:178-181

Montesi L G J, Zuber M T (2003) Clues to the lithospheric structure of Mars from wrinkle ridge sets and localization instability. J Geophys Res Planets 108:1-22

Montmerle T, Augereau J-C, Chaussidon M, Gounelle M, Marty B, Morbidelli A (2006) 3. Solar System formation and early evolution: the first 100 million years. Earth Moon Planets 98:39-95

Moreira M, Breddam K, Curtice J, Kurz M D (2001) Solar neon in the Icelandic mantle: new evidence for an undegassed lower mantle. Earth Planet Sci Lett 185:15-23

Morbidelli A, Chambers J, Lunine J I, Petit J-M, Robert F, Valsecchi G B, Cyr K E (2000) Source regions and timescales for the delivery of water to the earth. Meteoritics \& Planetary Sci 35:1309Ú 1320

Morbidelli A, Crida A (2007), The dynamics of Jupiter and Saturn in the gaseous protoplanetary disk. Icarus 191:158-171

Morbidelli A, Tsiganis K, Crida A, Levison H, Gomes R (2007), Dynamics of the giant planets of the Solar System in the gaseous protoplanetary disk and their relationship to the current orbital architecture. Astrophys J 134:1790-1798

Morbidelli A, Bottke W F, Nesvorný D, Levison H F (2009) Asteroids were born big. Icarus 204:558-573 
Morbidelli A, Brasser R, Gomes R, Levison H F, Tsiganis K (2010) Evidence from the asteroid belt for a violent past evolution of JupiterŠs orbit. Astron J 140:1391-1401

Morbidelli A, Lunine J I, O'Brien D P, Raymond S N, Walsh K (2012) Building terrestrial planets. Annu Rev Earth Planet Sci 40:251-275

Moynier F, Yin Q-Z, Irisawa K, Boyet M, Jacobsen B, Rosing M T (2010) Coupled ${ }^{182}$ W- ${ }^{142}$ Nd constraint for early Earth differentiation. PNAS 107:10810-10814

Mullally F, Coughlin J L, Thompson S E, Rowe J, Burke C, and the Kepler Team (2015) Planetary candidates observed by Kepler. VI. planet sample from Q1-Q16 (47 months). Astrophys J Supp 217:31, 16 pp

Murthy V R, Westrenen W van, Fei Y (2003) Experimental evidence that potassium is a substantial radioactive heat source in planetary cores. Nature 423:163-165

Mysen B (2017) Water-melt interaction in hydrous magmatic systems at high temperature and pressure. Prog Earth Planet Sci 1:1-18

Navarro-González R, McKay C P, Mvondo D N (2001) A possible nitrogen crisis for Archaean life due to reduced nitrogen fixation by lightning. Nature 412:61-64

Neumann W, Breuer D, Spohn T (2014) Differentiation of vesta: Implications for a shallow magma ocean. Earth Planet Sci Lett 395:267Û́280

Nicklas R W, Puchtel I S, Ash R D (2016) High-precision determination of the oxidation state of komatiite lavas using vanadium liquid-mineral partitioning. Chemical Geology 433:36-45

Nicklas R W, Puchtel I S, Ash R D (2018) Redox state of the Archean mantle: Evidence from V partitioning in 3.5-2.4 Ga komatiites. Geochim. Cosmochim. Acta 222:447-466

Noack L, Breuer D, Spohn T. (2012) Coupling the atmosphere with interior dynamics: implications for the resurfacing of Venus. Icarus 217(2), 484-498

Noack L, Breuer D (2014) Plate tectonics on rocky exoplanets: Influence of initial conditions and rheology. Planet Space Sci 98, 41-49

Nutman A P, Friend C R L, Bennett V C (2002) Evidence for 3650-3600 Ma assembly of the northern end of the Itsaq Gneiss Complex, Greenland: implication for early Archean tectonics. Tectonics 21:1005

Nutman A P, Bennett V C, Friend C R L, van Kranendonk M J, Chivas A R (2016) Rapid emergence of life shown by discovery of 3.700-million-year-old microbial structures. Nature 537:535-538

O’Brien D P, Morbidelli, A, Levison H F (2006) Terrestrial planet formation with strong dynamical friction. Icarus 184:39-58

O'Brien, D P, Walsh K J, Morbidelli A, Raymond S N (2014) Water delivery and giant impacts in the 'Grand Tack' scenario. Icarus 239:74-84

O'Neill C O, Jellinek A M, Lenardic A (2007) Conditions for the inset of plate tectonics on terrestrial planets and moons. Earth Planet Sci Lett 261:20-32

O'Neill H St C, Palme H (2017) Collisional erosion and the non-chondritic composition of the terrestrial planets. Phil Trans R Soc A 366:4205-4238

Odert P, Lammer H, Erkaev N V, Nikolaou A, Lichtenegger H I M, Johnstone C P, Kislyakova K G, Leitzinger $\mathrm{M}$, Tosi N (2018) Escape and fractionation of volatiles and noble gases from Mars-sized planetary embryos and growing protoplanets. Icarus in press

Ohtani E, Maeda M (2001) Density of basaltic melt at high pressure and stability of the melt at the base of the lower mantle. Earth Planet Sci Lett 193:69-75

Olson S L, Reinhard C T, Lyons T W (2016) Limited role for methane in the mid-Proterozoic greenhouse. PNAS 113:11447-11452

Owen T, Mahaffy P R, Niemann H B, Atreya S, Wong (2001) Protosolar nitrogen. Astrophys J 553:L77-L79

Owen J E, Mohanty S (2016) Habitability of terrestrial-mass planets in the HZ of M Dwarfs - I. H/Hedominated atmospheres. MNRAS 459:4088-4108

Owen J E, Wu Y (2016) Atmospheres of low-mass planets: the 'boil-off'. Astrophys J 817:107, 14 pp

Oyama V I, Carle G C, Woeller F, Pollack J B, Reynolds R T (1980) Pioneer Venus gas chromatography of the lower atmosphere of Venus. J Geophys Res 85:7891-7902

Pallavicini R, Golub L, Rosner R, Vaiana G S, Ayres T, Linsky J L (1981) Relations among stellar X-ray emission observed from Einstein, stellar rotation and bolometric luminosity. Astrophys J 248:279-290

Palma R L, Becker R H, Pepin R O, Schlutter D J (2002) Irratiaon records in regolth materials, II: Solar wind and solar energetic particle components in helium, neon, and argon extracted from single lunar mineral grains and from the Kapoeta howardite by stepwise pulse heating. Geochimica Cosmochimica Acta 66:2929-2958

Palumbo A M, Head III J W, Wordsworth R D (2018) Late Noachian icy highlands climate model: Exploring the possibility of transient melting and fluvial/lacustrine activity through peak annual and seasonal temperatures. Icarus 2018:261-286

Parai R, Mukhopadhyay S (2012) How large is the subducted water flux? New constraints on mantle regassing rates. EPSL 317-318, 396-406 
Pierens A, Nelson R P (2008) Constraints on resonant-trapping for two planets embedded in a protoplanetary disc. Astron Astrophys 482:333-340

Pizzolato N, Maggio A, Micela G, Sciortino S, Ventura P (2003) The stellar activity-rotation relationship revisited: Dependence of saturated and non-saturated X-ray emission regimes on stellar mass for late-type dwarfs. Astron Astrophys 397:147-157

Pearson D G, Brenker F E, Nestola F, McNeill J, Nasdala L, Hutchinson M T, Matveev S, Mather K, Silversmit G, Schmitz S, Vekemans B, Vincze L (2014) Hydrous mantle transition zone indicated by ringwoodite included within diamond. Nature 507:221-224

Pepin R O (1991) On the origin and early evolution of terrestrial planet atmospheres and meteoritic volatiles. Icarus 92:2-79

Pepin R O (1994) Evolution of the martian atmosphere. Icarus 111:289-304

Pepin R O (1997) Evolution of EarthŠs noble gases: consequences of assuming hydrodynamic loss driven by giant impact. Icarus 126:148-156.

Pepin R O (2000) On the isotopic composition of primordial xenon in terrestrial planet atmospheres. Space Sci. Rev. 92:371-395

Pepin R O, Porcelli D (2002) Origin of noble gases in the terrestrial planets. In: Porcelli D, Ballentine C J, Wieler R (eds) Noble Gases in Geochemistry and Cosmochemistry. Rev Mineral Geochem 47, Mineralogical Society of America, Washington DC, pp 191-246

Pham L B S, Karatekin Ö, Dehant V (2011) Effects of impacts on the atmospheric evolution: Comparison between Mars, Earth and Venus. Planet Space Sci 59:1087-1092

Pierrehumbert R, Gaidos E (2011) Hydrogen greenhouse planets beyond the habitable zone. Astrophys J Lett 734:L13, $5 \mathrm{pp}$

Plesa A-C, Tosi N, Breuer D (2014) Can a fractionally crystallized magma ocean explain the thermo-chemical evolution of mars? Earth Planet Sci Lett 403:225Ú235

Plümper O, John T, Podladchikov Yu Y, Vrijmoed J C, Scambelluri M (2017) Fluid escape from subduction zones controlled by channel-forming reactive porosity. Nature Geoscience 10:150-156

Pollacco D L, Skillen I, Collier Cameron A, Christian D J, Hellier C, Irwin J, Lister T A, Street R A, West R G, Anderson D R, Clarkson W I, Deeg H, Enoch B, Evans A, Fitzsimmons A, Haswell C A, Hodgkin S, Horne K, Kane S R, Keenan F P, Maxted P F L, Norton A J, Osborne J, Parley N R, Ryans R S I, Smalley B, Wheatley P J, Wilson D M (2006) The WASP project and the superWASP cameras Pub Astron Soc Pacific 118:1407-1418

Pollack J B, Hubickyj O, Bodenheimer P, Lissauer J J, Podolak M, Greenzweig Y (1996) Formation of the Giant Planets by Concurrent Accretion of Solids and Gas. Icarus 124:62-85

Porcelli D, Pepin R O (2000) Rare gas constraints on early Earth history. In: Canup R M, Righter K (eds) Origin of the Earth and Moon. University of Arizona Press, Tucson, pp. 435-458

Porcelli D, Pepin R O (2003) The origin of noble gases and major volatiles in the terrestrial planets. In: Holland H D, Turekian K K, (eds) The atmosphere. Treatise on Geochemistry 4:319-347

Porcelli D, Cassen P, Woolum D, Wasserburg G J (1998) Acquisition and early losses of rare gases from the deep Earth, in: Origin of the Earth and Moon. Lunar and Planetary Inst, 597:55-36

Porcelli D, Cassen P, Woolum D (2001) Deep Earth rare gases: initial inventories, capture from the solar nebula and losses during Moon formation. Earth Planet Sci Lett 193:237-251

Poulton S W, Canfield D E (2011) Ferruginous conditions: A dominant feature of the ocean through Earth's history. Elements 7:107-12

Pujol M, Marty B, Burnard P, Philippot P (2009) Xenon in Archean barite. Geochimica Cosmochimica Acta Supplement 73, A1059

Pujol M, Marty B, Burgess (2011) Chondritic-like xenon trapped in Archean rocks: A possible signature of the ancient atmosphere. Earth Planet. Sci. 308:298-306

Ramirez R M, Kopparapu R, Zugger M E, Robinson T D, Freedman R, Kasting J F (2014) Warming early Mars with $\mathrm{CO}_{2}$ and $\mathrm{H}_{2}$. Nature Geosci 7:59-63

Ranero C R, Morgan J P, McIntosh K, Reichert C (2003) Bending-related faulting and mantle serpentinization at the Middle America trench. Nature 425:367-73

Rasool S I, de Bergh C (1970) The runaway greenhouse effect and the accumulation of $\mathrm{CO}_{2}$ in the atmosphere of Venus. Nature 226:1037-1039

Raymond S N, Quinn T, Lunine J I (2004) Making other Earths: dynamical simulations of terrestrial planet formation and water delivery. Icarus 168:1-17

Raymond S N, Quinn T, Lunine J I (2005) Terrestrial planet formation in disks with varying surface density profiles. Astrophys J 632:670-676

Raymond S N, Quinn T, Lunine J I (2006) High-resolution simulations of the final assembly of Earth-like planets I. terrestrial accretion and dynamics. Icarus 183:265-282 
Raymond S N, Quinn T, Lunine J I (2007) High-resolution simulations of the final assembly of Earth-like planets. 2. Water delivery and planetary habitability. Astrobiology 7:66-84

Raymond S N, O'Brien D P, Morbidelli A, Kaib N A (2009) Building the terrestrial planets: constrained accretion in the inner solar system. Icarus 203:644-662

Raymond S N, Morbidelli A (2014) The Grand Tack model: a critical review, complex planetary systems. Proc Int Astron Union, IAU Symposium, 310:194-203

Raymond S N, Kokubo E, Morbidelli A, Morishima R, Walsh K J (2014) Terrestrial planet formation at home and abroad. In: Beuther H, Klessen R S, Dullemond C P, Henning T (eds) Protostars and Planets VI. Univ Arizona Press, Tucson, pp 595-618

Reese C C, Solomatov V S (2006) Fluid dynamics of local martian magma oceans. Icarus 184:102Ú120

Regenauer-Lieb K, Yuen D A, Branlund J (2001) The initiation of subduction: Criticality by addition of water? Science 294:578-581

Reinhard C T, Planavsky N J (2011) Mineralogical constraints on Precambrian $\mathrm{pCO}_{2}$. Nature 474:E4-5

Ribas I, Guinan EF, Güdel M, Audard M (2005) Evolution of the solar activity over time and effects on planetary atmospheres: I. High-energy irradiances (1-1700 ̊̊). ApJ 622:680

Robbins S J, Hynek B M, Lillis R J, Bottke W F (2013) Large impact crater histories of Mars: The effect of different model crater age techniques. Icarus 225:173-184

Roberson A L, Roadt J, Halevy I, Kasting J F (2011) Greenhouse warming by nitrous oxide and methane in the Proterozoic Eon. Geobiology 9:313-20

Rodriguez J A P, Fairén A G, Tanaka K L, Zarroca M, Linares R, Platz T, Komatsu G, Miyamoto H, Kargel J S, Yan J, Gulick V, Higuchi K, Baker V R, Glines N (2015) Tsunami waves extensively resurfaced the shorelines of an early Martian ocean. Nature Sci Reports 6:25106

Rogers L A (2015) Most 1.6 Earth-radius planets are not rocky. Astrophys J 801:41, 13 pp

Ronov A B, Yaroshevskiy A A (1967) Chemical structure of the Earth's crust. Geochemistry 11:1041-1066

Rosenbauer E N, Head III J W (2015) Late Noachian fluvial erosionon Mars:Cumulative water volumes required to carve the valley networks and grain size of bed-sediment. Planet Space Sci 117:429-435

Rosing M T, Frei R (2004) U-rich Archaean sea-floor sediments from Greenland - indications of > $3700 \mathrm{Ma}$ oxygenic photosynthesis. Earth Planet Sci Lett 217:237-244

Rosing M T, Bird D K, Sleep N H, Bjerrum C J (2010a) No climate paradox under the faint young Sun. Nature 464:744-747

Rosing M T, Bird D K, Sleep N H, Bjerrum C J (2010b) Rosing, Bird, Sleep \& Bjerrum reply. Nature 474, E1

Rubie D C, Nimmo F, Melosh H J (2015) Formation of the Earth's core. (ed Schubert G) Treatise on Geophysics 9:43Ü79

Rye R, Kuo P H, Holland H D (1995) Atmospheric carbon dioxide concentrations before 2.2 billion years ago. Nature 378:603-605

Sackmann I J, Boothroyd A I (2003) Our Sun. V. A bright young Sun consistent with helioseismology and warm temperatures on ancient Earth and Mars. Astrophys J 583:1024-1039

Salvador A, Massol H, Davaille A, Marcq E, Sarda P, Chassefiére E (2017) The relative influence of $\mathrm{H}_{2} \mathrm{O}$ and $\mathrm{CO}_{2}$ on the primitive surface conditions and evolution of rocky planets. J Geophys Res Planets 122:1458Ü1486

Sasaki S, Nakazawa K (1988) Origin of isotopic fractionation of terrestrial Xe: hydrodynamic fractionation during escape of the primordial $\mathrm{H}_{2}$ and $\mathrm{He}$ atmosphere. Earth Planet Sci Lett 89:323-334

Sasaki S, Nakazawa K (1989) did a primary solar-type atmosphere exist around the proto-Earth? Icarus $85: 21-42$

Safronov V S (1969) Evolution of the protoplanetary cloud and formation of the Earth and the planets. Akad Nauk SSSR Moscow, English translation, NASA TTF-667, 1972

Safronov V S (1972) Evolution of the protoplanetary cloud and formation of the Earth and planets (Ed Safronov V S). Translated from Russian. Jerusalem (Israel): Israel Program for Scientific Translations, Keter Publishing House, 212 pp.

Schaefer L, Fegley B (2010) Chemistry of atmospheres formed during accretion of the Earth and other terrestrial planets. Icarus 208:438-448

Sheldon (2006) Precambrian paleosols and atmospheric $\mathrm{CO}_{2}$ levels. Precambrian Research 147:148-155

Scherf M, Khodachenko M L, Blokhina M, Johnstone C, Alexeev I, Belenkaya E, Tarduno J A, Kulikov Yu N, Tu L, Lichtenegger H I M, Güdel M, Lammer H (2017) On the Earth's paleo-magnetosphere during the late Hadean eon and possible implications for the ancient terrestrial atmosphere. Earth Planet Sci Lett, in preparation

Schlesinger W H (1997) Biogeochemical cycles. Biogeochemistry, Academy Press

Schmandt B, Jacobsen S D, Becker T W, Liu Z, Dueker K G (2014) Dehydration melting at the top of the lower mantle. Science 344:165-1268 
Segura T L., Toon O B, Colaprete A, Zahnle K (2002) Environment effects of large impacts on Mars. Science 298:1977-1980

Segura T L, Toon O B, Colaprete A (2008) Modeling the environmental effects of moderate-sized impacts on Mars

Segura T L, Mc Kay C P, Toon O B (2012) An impact-induced, stable climate on Mars

Sekiya M, Nakazawa K, Hayashi C (1980a) Dissipation of the rare gases contained in the primordial Earth's atmosphere. Earth Planet Sci Lett 50:197-201

Sekiya M, Nakazawa K, Hayashi C (1980b) dissipation of the primordial terrestrial atmosphere due to irradiation of the solar EUV. Prog Theoret Phys 64:1968-1985

Shirey S B, Kamber B S, Whitehouse M J, Mueller P A, Basu A R (2008) A review of the isotopic and trace element evidence for mantle and crustal processes in the Hadean and Archean: implications for the onset of plate tectonic subduction. In: Condie K C, Pease V (eds) When did plate tectonics begin on planet Earth? Geological Society of America, Boulder, pp 1-29

Skumanich A (1972) Time scales for CA II emission decay, rotational braking, and Lithium depletion. Astrophys J 171:565

Sleep N H, Zahnle K J, Kasting J F, Morowitz, H J (1989) Annihilation of ecosystems by large asteroid impacts on the early earth. Nature 342:139-142

Sleep N H, Zahnle K (1991) Carbon dioxide cycling and implecations for climate on ancient Earth. J Geophys Res 106:1373-1399

Sleep N H (1991) Hydrothermal circulation, anhydrite precipitation, and thermal structure at ridge axes. J Geophys Res 96:2375-2387

Sleep N H (2010) The Hadean-Archean environment. Cold Spring Harb Perspect Biol 2(6), a002527

Sleep N F, Zahnle K J, Lupu R E (2017) Terrestrial aftermath of the Moon-forming impact. Phil Trans Royal Soc A 372:20130172

Soderblom D R, Stauffer J R, MacGregor K B, Jones B F (1993) The evolution of angular momentum among zero-age main-sequence solar-type stars. Astrophs J 409:624-634

Solomatov V S (2000) Fluid dynamics of a terrestrial magma ocean. In: Canup R M, Righter K (eds) Origin of the Earth and Moon. Univ. Arizona Press, Tucson pp 323-338

Solomatov V S (2004) Initiation of subduction by small-scale convection. J Geophys Res 109:B01412

Som S M, Buick R, Hagadorn J W, Blake T S, Perreault J M, Harnmeijer J P, Catling D C (2016) Earth's air pressure 2.7 billion years ago constrained to less than half of modern levels. Nature Geoscience 484:359-362

Srinivasan B (2016) Barites - Anomalous xenon from spallation and neutron-induced reactions. Nature Geoscience 31:129-141

Stanley B D, Hirschmann M M, Withers A C (2011) $\mathrm{CO}_{2}$ solubility in Martian basalts and Martian atmospheric evolution. Geochim Cosmochim Acta 75:5987-6003

Stauffer J R, Caillault J-P, Gagné M., Prosser C F, Hartmann L W (1994) A deep imaging survey of the Pleiades with ROSAT. Astrophys J Supp 91:625-657

Stern R J (2005) Evidence from ophiolites, blueschists, and ultrahigh-pressure metamorphic terranes that the modern episode of subduction tectonics began in Neoproterozoic time. Geology 33:557-60

Stevenson D J (1983a) Anomalous bulk viscosity of two-phase fluids and implications for planetary interiors. J Geophys Res 88:2445-2455

Stevenson D J (1983b) The nature of the Earth prior to the oldest known rock record: The Hadean Earth. In: Earth's earliest biosphere: Its origin, and evolution (Schopf J W ed), Princeton University Press, p 32-40

Stevenson D J (1990) Fluid dynamics of core formation. In: Stevenson, D.J. Fluid dynamics of core formation. In: Newsom H E, Jones J H (eds) Origin of the Earth. Oxford University Press, pp 231-249

Stökl A, Dorfi E, Lammer H (2015) Hydrodynamic simulations of captured protoatmospheres around Earthlike planets. Astron Astrophys 576:87, $11 \mathrm{pp}$

Stökl A, Dorfi E A, Johnstone C P, Lammer H (2016) Dynamical accretion of primordial atmospheres around planets with masses between 0.1 and $5 M_{\oplus}$ in the habitable zone. Astrophys J 825:86, $11 \mathrm{pp}$

Strom R G, Schaber G G, Dawson D D (1994) The global resurfacing of Venus. J Geophys Res 99:10899-10926

Stüeken E E, Buick R, Schauer A J (2015) Nitrogen isotope evidence for alkaline lakes on late Archean continents. Earth and Planetary Science Letters 411: 1-10

Stüeken E E, Kipp M A, Koehler M C, Schwieterman E W, Johnson B, Buick R (2016) Modeling pN 2 through geological time: Implications for planetary climates and atmospheric biosignatures. Astrobiology 16:949963

Stüeken E E, Kipp M A, Koehler M C, Buick R (2016a) The evolution of Earth's biogeochemical nitrogen cycle. Earth Sci Rev 160:220-39

Tang M, Chen K, Rudnick, R L (2016) Archean upper crust transition from mafic to felsic marks the onset of plate tectonics. Science 372:375 
Tashiro T, Early trace of life from 3.95 Ga sedimentary rocks in Labrador, Canada. Nature 549:516-518

Tarduno J A, Blackman E G, Mamajek E E (2014) Detecting the oldest geodynamo and attendant shielding from the solar wind: Implications for habitability. Phys Earth Planet Int 233:68-87

Tarduno J A, Cottrell R T, Davis W J, Nimmo F, Bono R K (2015) A Hadean to Paleoarchean geodynamo recorded by single zircon crystals. Science 349:521-524

Thauer R K (1998) Biochemistry of methanogenesis: Á tribute to marjory stephenson. marjory stephenson prize lecture. Microbiology 144:2377-2406

Tian F, Toon O B, Pavlov A A, De Sterck H (2005) A hydrogen-rich early Earth atmosphere. Science 308:1014-1017

Tian F, Kasting J F, Liu H-L, Roble R G (2008a) Hydrodynamic planetary thermosphere model: 1. Response of the Earth's thermosphere to extreme solar EUV conditions and the significance of adiabatic cooling. J Geophys Res 113, E05008

Tian F, Solomon S C, Qian L, Lei J, Roble R G (2008b) Hydrodynamic planetary thermosphere model: 2. Coupling og an electron transport/energy deposition model. J Geophys Res 113, E07005

Tian F, Kasting J F, Solomon S C (2009) Thermal escape of carbon from the early Martian atmosphere. Geophys Reas Lett 36, L02205

Tian F, Kasting JF, Zahnle K. 2011. Revisiting HCN formation in Earth's early atmosphere. Earth and Planetary Science Letters 308: 417-23

Thomazo C, Papineau D (2013) Biogeochemical cycling of nitrogen on the early Earth. Elements 9:345-351

Tosi N, Plesa A-C, Breuer D (2013) Overturn and evolution of a crystallized magma ocean: a numerical parameter study for Mars. J Geophys Res Planets 118:1512Û1528

Tsiganis K, Gomes R, Morbidelli A, Levision H F (2005), Origin of the orbital architecture of the giant planets of the Solar System. Nature 435:459-461

Tu L, Johnstone C P, Gd̈el M, Lammer H (2015) The extreme ultraviolet and x-ray Sun in time: High-energy evolutionary tracks of a solar-like star. Astron Astrophys 577 L3

Tucker J M , Mukhopadhyay S (2014) Evidence for multiple magma ocean outgassing and atmospheric loss episodes from mantle noble gases. EPSL 393, 254-265

Turcotte D L, Schubert G (1982) Geodynamics: Applications of continuum physics to geological problems. Wiley, New York

Turner G, Burgess R, Bannon M (1990) Volatile-rich mantle fluids inferred from inclusions in diamond and mantle xenoliths. Nature 344:653-655

Turner S, Rushmer T, Reagan M, Moyen J-F (2014) Heading down early on? Start of subduction on early Earth. Geology 42:139-42

Tyburczy J A, Frisch B, Ahrens T J (1986) Shock-induced volatile loss from a carbonaceous chondrite: Implications for planetary accretion. Earth Planet Sci Lett 80:201-207

Urey H C (1955) The cosmic abundances of potassium, uranium, and thorium and the heat balance of the Earth, the Moon, and Mars. PNAS 41:127-44

Valley J W, Peck W H, King E M, Wilde S A (2002) A cool early Earth. Geology 30:351-355

Valkiers S, Aregbe Y, Taylor P, De Bièvre (1998) A primary xenon isotopic gas standard with SI traceable values for isotopic composition and molar mass. Int. J. Mass Spectrom. Ion Process. 173:55-63

Van Kranendonk M J, Smithies R H, Hickman A H, Champion D (2007) Review: secular tectonic evolution of Archean continental crust: Interplay between horizontal and vertical processes in the formation of the Pilbara Craton, Australia. Terra Nova 19:1-38

Van Kranendonk M J (2010) Two types of Archean continental crust: Plume and plate tectonics on early Earth. Am J Sci 310:1187-1209

Van Kranendonk M J (2011) Onset of plate tectonics. Science 333:413-414

Von Paris P, Rauer H, Lee Grenfell J, Patzer B, Hedelt P, Stracke B, Trautmann T, Schreier F (2008) Warming the early EarthÜCO 2 reconsidered. Planet Space Sci 56:1244-1259

Wahl S M, Hubbard W W, Militzer B, Guillot T, Miguel Y, Movshovitz N, Kaspi Y, Helled R, Reese D, Galanti E, Levin S, Connerney J E, Bolton S J (2017) Comparing Jupiter interior structure models to Juno gravity measurements and the role of a dilute core. Geophys Res Lett 44:4649-4659

Walker D, Longhi J, Hays J F (1975) Differentiation of a very thick magma body and implications for the source region of mare basalts. Lunar Planet Inst, Proc Lunar Sci Conf, 6th Houston Texas March 17-21, 1103-1120

Walker J C G (1977) Evolution of the atmosphere. Macmillan, New York

Walker J C G (1985) Carbon dioxide on the early Earth. Origins Life 16:117-127

Walsh K J, Morbidelli A, Raymond S N, O’Brien D P, Mandell A M (2011) A low mass for Mars from Jupiter's early gas-driven migration. Nature 475:206-209

Walter F M (1981) On the coronae of rapidly rotating stars. II - A period-activity relation in G stars. Astrophys J 245:677-681 
Wang Z, Becker H (2013) Ratios of S, Se and Te in the silicate Earth require a volatile-rich late veneer. Nature 499:328-331

Watenphul A, Wunder B, Wirth R, Heinrich W (2010) Ammonium-bearing clinopyroxene: A potential nitrogen reservoir in the Earth's mantle. Chem Geol 270:240-248

Watson A J, Donahue T M, Walker J C G (1981) The dynamics of a rapidly escaping atmosphere: Applications to the evolution of Earth and Venus. Icarus 48:150-166

Weber E J, Davis Jr L (1967) The angular momentum of the solar wind. Astrophys J 148:217-227

Wieler R (2016) Do lunar and meteoritic archives record temporal variations in the composition of solar wind noble gases and nitrogen? A reassessment in the light of Genesis data. Chemie der Erde 76:463-480

Wieler R, Heber V S (2003) Noble gas isotopes on the Moon. Space Sci Rev 106:197-211

Way M J, Del Genio A D, Kiang N Y, Sohl L E, Grinspoon D H, Aleinov I, Kelley M, Clune T (2016) Was Venus the first habitable world of our solar system? Geophys. Res. Lett. 43, 8376-8383

Weiss M C, Sousa F L, Mrnjavac N, Neukirchen S, Roettger M, Nelson-Sathi S, Martin W F (2016) The physiology and habitat of the last universal common ancestor. Nat Microbiol 1(9):16116

Wetherill G W (1980) Formation of the terrestrial planets. Annu Rev Astron Astrophys 18:77-113

Wetherill G W (1991) Occurrence of earth-like bodies in planetary systems. Science 253:535-538

Wilde S A, Valley J W, Peck W H, Graham C M (2001) Evidence from detrital zircons for the existence of continental crust and oceans on the Earth 4.4 Gyr ago. Nature 409:175-178

Wolf E T, Toon O B (2013) Hospitable Archean climates simulated by a general circulation model. Astrobiology 13:656Ú673

Wood B J, Walter M J, Wade J (2006) Accretion of the Earth and segregation of its core. Nature 441:825-833

Wordsworth R D, Pierrehumbert R T (2013) Water loss from terrestrial planets with $\mathrm{CO}_{2}$-rich atmospheres. Astrophys J 778:154, 19 pp

Wordsworth R D, Pierrehumbert R T (2014) Abiotic oxygen-dominated atmospheres on terrestrial habitable zone planets. Astrophys J Lett 785:2 L20

Wordsworth R D (2016a) Atmospheric nitrogen evolution on Earth and Venus. Earth Planet Sci Lett 447:103111

Wordsworth R D (2016b) The Climate of Early Mars. Annual Rev Earth Planet Sci 44:1-31

Wright N J, Drake J J, Mamajek E E, Henry G W (2011) The stellar-activity-rotation relationship and the evolution of stellar dynamos. Astrophys J 743:48, 16 pp

Wyllie P J, Ryabchikov D, (2000) Volatile components, magmas, and critical fluids in upwelling mantle. J Petrology 41:1195-1206

Yin A (2012) "Structural analysis of the Valles Marineris fault zone: Possible evidence for large-scale strikeslip faulting on Mars". Lithosphere 4:286-330

Yin Q, Jacobsen S B, Yamashita K, Blichert-Toft J Télouk P, Albaréde F (2002) A short timescale for terrestrial planet formation from Hf-W chronometry of meteorites. Nature, Volume 418, Issue 6901, pp. 949-952

Yokochi R, Marty B (2004) A determination of the neon isotopic composition of the deep mantle. Earth Planet Sci Lett 225:77-88

Yu G, Jacobsen S B (2011) Fast accretion of the Earth with a late Moon-forming giant impact. PNAS 108:17604-17609

Zahnle K J, Walker J C G (1982) The evolution of solar ultraviolet luminosity. Rev. Geophys. 20:280-292.

Zahnle K, Kasting J F, Pollack J B (1986) Mass fractionation of noble gases in diffusion-limited hydrodynamic hydrogen escape. Icarus 84:502-527

Zahnle K J (1986) Photochemistry of methane and the formation of hydrocyanic acid (HCN) in th earth's early atmosphere. Journal of Geophysical Research 91: 2819-2834

Zahnle K, Pollack J B, Kasting J F (1990) Xenon fractionation in porous planetesimals. Geochim Cosmochim Acta 54:2577-2586

Zahnle K J, Claire M W, Catling D C (2006) The loss of mass-independent fractionation in sulfur due to a Palaeoproterozoic collapse of atmospheric methane. Geobiology 4:271-283

Zahnle K, Haberle R M, Catling D C, Kasting J F (2008) Photochemical instability of the ancient Martian atmosphere. J Geophys Res 113:E11004

Zahnle K, Freedman R, Catling D (2010) Is there methane on Mars? 41st Lunar Planet Sci Conf, March 1-5, 2010, The Woodlands, Texas, 1533, p 2456

Zahnle K J (2015) Xenon fractionation and Archean hydrogen escape. Lunar Planet. Sci. XLVI 1549 (abstract).

Zerkle A L, Junium C K, Canfield D E, House C H (2008) Production of ${ }^{15} \mathrm{~N}$-depleted biomass during cyanobacterial $\mathrm{N}_{2}$-fixation at high Fe concentrations. J Geophys Res 113:G03014

Zerkle A L, Mikhail S (2017) The geobiological nitrogen cycle: From microbes to the mantle. Geobiology, in press, DOI: $10.1111 / \mathrm{gbi} .12228$ 
Zerkle A L, Poulton S W, Newton R J, Mettam C, Clair M W, Bekker A, Junium C K (2017) Onset of the aerobic nitrogen cycle during the Great Oxidation Event. Nature 542: 465-467 